\title{
The Influence of Redox-Active Linkers on the Stability and Physical Properties of a Highly Electroactive Porphyrin Nanoprism
}

Kaitlyn G. Dutton, Daniel A. Rothschild, Dakota B. Pastore, Thomas J. Emge, and Mark C. Lipke*

Department of Chemistry and Chemical Biology, Rutgers The State University of New Jersey

123 Bevier Road, Piscataway NJ 08854

ml1353@chem.rutgers.edu

Supporting Information

$\underline{\text { Table of Contents: }}$

1. General Considerations.

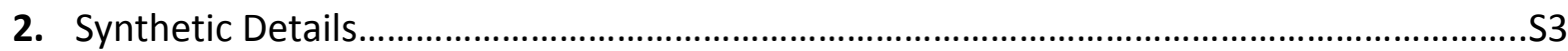

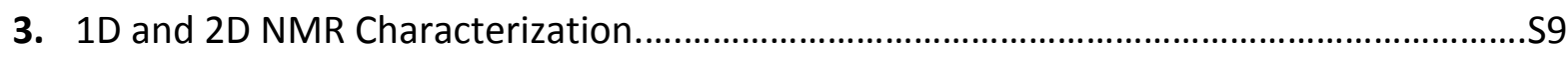

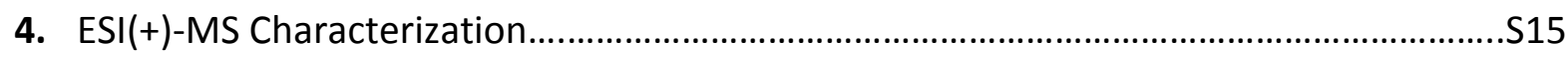

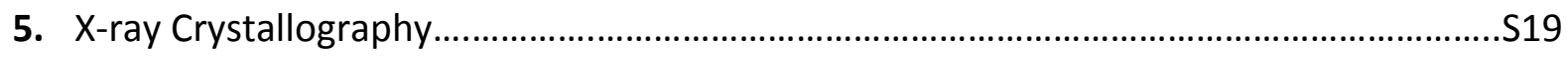

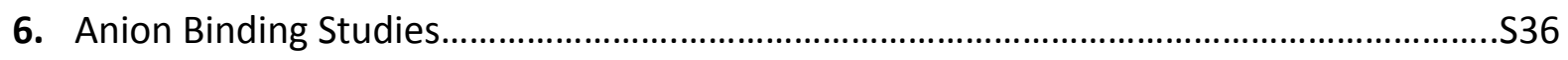

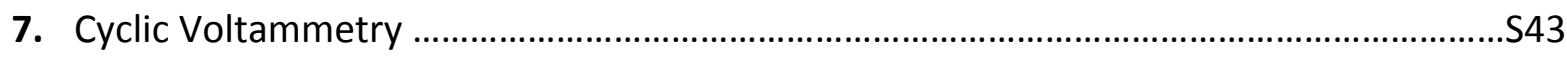

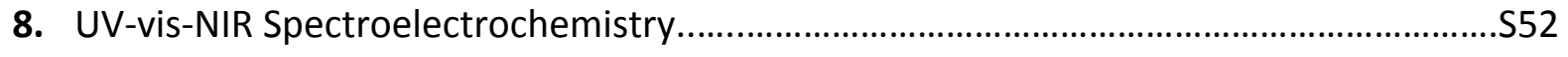

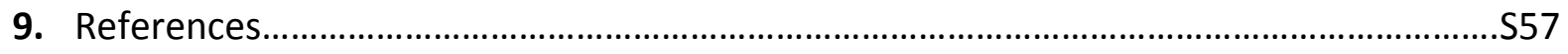




\section{General Considerations}

Sources: Commercially available chemicals and solvents were used as received unless otherwise specified - (1) from Fisher: acetonitrile, diethyl ether, potassium iodide, and pyridine; (2) from Alfa-Aesar: 2,2'-bipyridine, ferrocene, and $N, N, N^{\prime}, N^{\prime}$-tetramethylethylenediamine; (3) from Pressure Chemical: potassium tetrachloroplatinate; (4) from Acros Organics: tetrabutylammonium hexafluorophosphate, tetrabutylammonium hydrogen sulfate, acetone- $d_{6}$ (D-99\%), and silver trifluoromethanesulfonate; (5) from SynQuest Laboratories: ammonium hexafluorophosphate; (6) from Cambridge Isotopes: acetonitrile-d ${ }_{3}$ (D-99.8\%); (7) from SigmaAldrich: $\mathrm{N}, \mathrm{N}$-dimethylformamide; (8) from Oakwood Chemical: ammonium tetrafluoroborate; and (9) from Decon Labs Inc.: 200 proof absolute ethanol. Tetrakis(3-pyridyl)porphyrin (2), ${ }^{1}$ [tetrakis $\left(N\right.$-methyl-3-pyridinium)porphyrin] $\cdot 4 \mathrm{PF}_{6}(4),{ }^{2}\left[(\right.$ bipy $\left.) \mathrm{Pt}(\mathrm{py})_{2}\right] \cdot 2 \mathrm{PF}_{6}(\mathbf{5 b}),{ }^{3,4}$ and the $\mathrm{Pd}-$ based analogue ${ }^{5}$ of cage 1a were all prepared according to literature procedures. Synthetic procedures for (TMEDA)Ptl ${ }_{2},{ }^{6} \quad$ [(TMEDA)Pt(NCMe $\left.)_{2}\right] \cdot 2 \mathrm{OTf} \quad(3 \mathrm{3a}){ }^{6} \quad$ (bipy) $\mathrm{PtCl}_{2}{ }^{7}$ and $\left[\left(\right.\right.$ bipy)Pt( $\left.(\mathrm{NCMe})_{2}\right] \cdot 2 \mathrm{OTf}(\mathbf{3 b})^{7,8}$ were adapted from the literature. The adapted procedures are described in the Synthetic Details.

Purification: Dry, air-free solvents were obtained by sparging HPLC grade solvents with argon for $1 \mathrm{~h}$ before passage through solvent drying columns supplied by Pure Process Technologies. Acetonitrile for electrochemistry experiments was additionally dried over $3 \AA$ molecular sieves before being subject to 3 freeze-pump-thaw cycles and distillation via vacuum transfer. Ferrocene was purified by sublimation under vacuum prior to use. Tetrabutylammonium hexafluorophosphate was recrystallized 3 times from absolute ethanol and dried at $125^{\circ} \mathrm{C}$ under dynamic vacuum for $\geq 12 \mathrm{~h}$ before use as a supporting electrolyte. ${ }^{9}$

Physical Measurements: NMR spectra were recorded at $25{ }^{\circ} \mathrm{C}$ using a Bruker AVANCE Neo $500 \mathrm{MHz}$ spectrometer or a Varian VNMRS $500 \mathrm{MHz}$ spectrometer. Spectra were referenced using the residual proteo signal of the solvent for ${ }^{1} \mathrm{H} N M R$ data, the solvent ${ }^{13} \mathrm{C}\left\{{ }^{1} \mathrm{H}\right\} \mathrm{NMR}$ signal for ${ }^{13} \mathrm{C}\left\{{ }^{1} \mathrm{H}\right\}$ data, and a sealed capillary of fluorobenzene in $\mathrm{CD}_{3} \mathrm{CN}$ for ${ }^{19} \mathrm{~F} N \mathrm{NMR}$ data. High resolution mass spectra were obtained via direct injection on a Waters SYNAPT G2-SI qTOF mass spectrometer. Single-crystal X-ray diffraction data were collected on a Bruker diffractometer equipped with a Smart APEX CCD area detector. UV-vis-NIR spectra were recorded on samples in quartz cuvettes using a Shimadzu UV-2600i spectrophotometer equipped with an ISR-2600Plus integrating sphere attachment with a 220 - $1400 \mathrm{~nm}$ wavelength range for absorbance measurements. A CH Instruments $600 \mathrm{E}$ potentiostat was used for all electrochemical experiments. Specific details of physical measurements (e.g. sample preparation procedures) are described in their respective sections below. 


\section{Synthetic Details}

A. Cage 1a was prepared by the route depicted in Scheme S1. Procedures for the individual steps are described below.

\section{Scheme S1. Synthesis of Cage 1a.}

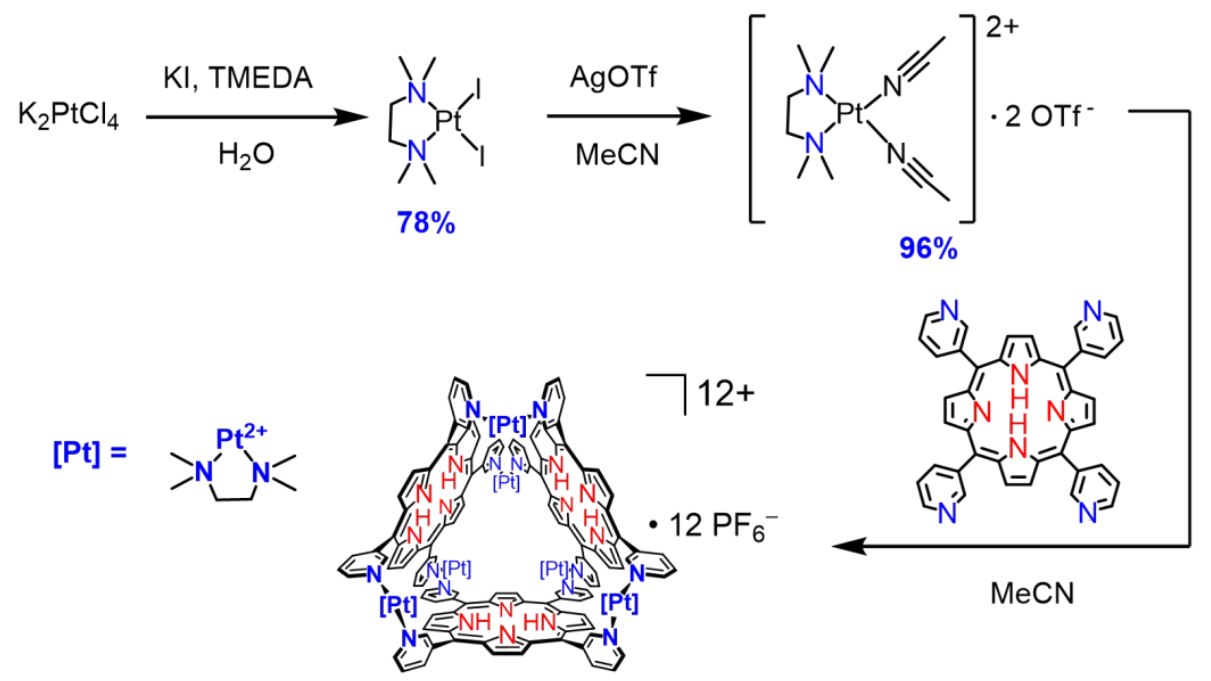

$1 \mathrm{a}$

$10 \%$

(TMEDA)Ptl ${ }_{2}$ was synthesized by a modified literature procedure ${ }^{6}$ :

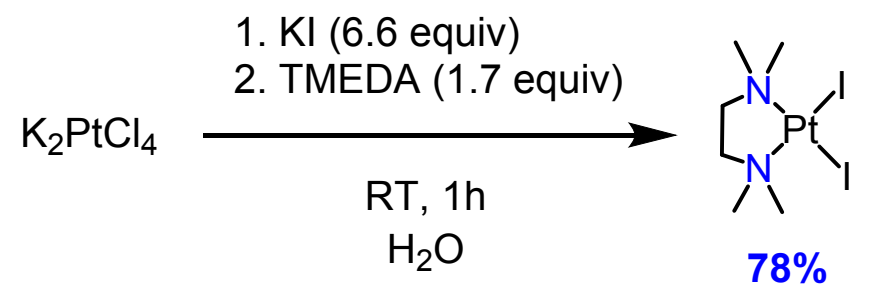

Potassium tetrachloroplatinate $\left(\mathrm{K}_{2} \mathrm{PtCl}_{4}, 0.641 \mathrm{~g}, 1.54 \mathrm{mmol}\right)$ and potassium iodide $(\mathrm{KI}, 1.702 \mathrm{~g}$, $10.26 \mathrm{mmol}$ ) were dissolved in $6 \mathrm{~mL}$ of deionized water and stirred in the dark for $30 \mathrm{~min}$, resulting in a dark red solution. A solution of $N, N, N^{\prime}, N^{\prime}$-tetramethylethylenediamine (TMEDA, $0.40 \mathrm{~mL}, 2.67 \mathrm{mmol}$ ) in $0.5 \mathrm{~mL}$ of water was added dropwise over $5 \mathrm{~min}$, and the mixture was stirred for an additional $30 \mathrm{~min}$, resulting in the precipitation of a yellow powder. The precipitate was collected by vacuum filtration and washed sparingly with acetone to remove brown-green impurities. The product was further washed with $6 \mathrm{~mL} \mathrm{H} \mathrm{H}_{2} \mathrm{O}, 6 \mathrm{~mL} \mathrm{EtOH}$, and $6 \mathrm{~mL} \mathrm{Et}_{2} \mathrm{O}$ before drying in air to yield $0.629 \mathrm{~g}$ of a fine yellow powder that was poorly soluble in most common solvents (78\% yield). ${ }^{1} \mathrm{H}$ NMR $\left(500 \mathrm{MHz},\left(\mathrm{CD}_{3}\right)_{2} \mathrm{CO}\right): \delta 3.07(\mathrm{~s}, J(\mathrm{Pt}-\mathrm{H})=37 \mathrm{~Hz}, 12 \mathrm{H}), 2.78(\mathrm{~s}, J(\mathrm{Pt}-$ $\mathrm{H})=24 \mathrm{~Hz}, 4 \mathrm{H})$. 
[(TMEDA)Pt(NCMe) 2 ]-20Tf (3a) was synthesized by a modified literature procedure ${ }^{6}$ :

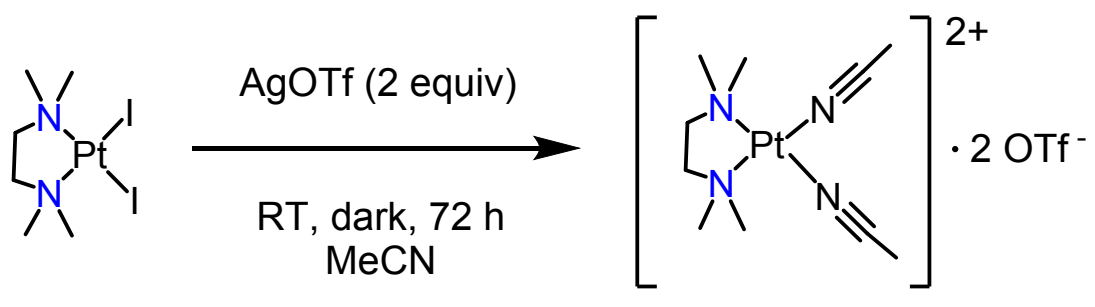

$3 a$

\section{$96 \%$}

[TMEDA]PtI 2 (0.629 g, $1.11 \mathrm{mmol}$ ) and silver trifluoromethanesulfonate (AgOTf, $0.572 \mathrm{~g}, 2.23$ $\mathrm{mmol}$ ) were combined in $40 \mathrm{~mL}$ of $\mathrm{MeCN}$ to provide a yellow suspension that was stirred for $72 \mathrm{~h}$ at room temperature with careful exclusion of light. A very-pale yellow precipitate of $\mathrm{Agl}$ formed over this time and was removed by filtration through a cellulose filter followed by a 0.2 $\mu \mathrm{m}$ PTFE syringe filter. The resulting clear, colorless filtrate was evaporated under reduced pressure to provide $0.740 \mathrm{~g}$ of $3 \mathrm{a}$ as a white solid (96\% yield). ${ }^{1} \mathrm{H} \mathrm{NMR}\left(500 \mathrm{MHz}, \mathrm{CD}_{3} \mathrm{CN}\right.$ ) $\delta 2.97$ $(\mathrm{s}, J(\mathrm{Pt}-\mathrm{H})=34 \mathrm{~Hz}, 12 \mathrm{H}), 2.90(\mathrm{~s}, J(\mathrm{Pt}-\mathrm{H})=23 \mathrm{~Hz}, 4 \mathrm{H}), 2.60(\mathrm{~s}, 6 \mathrm{H})$.

\section{Cage 1a}

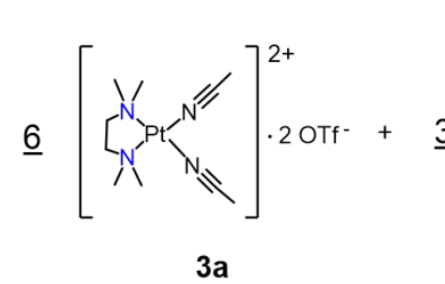

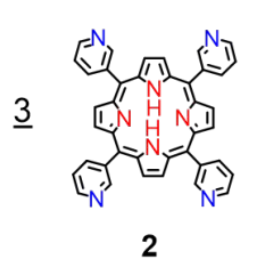

2

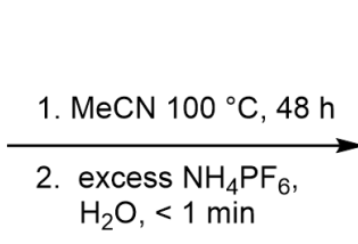

$\mathrm{H}_{2} \mathrm{O},<1$ min

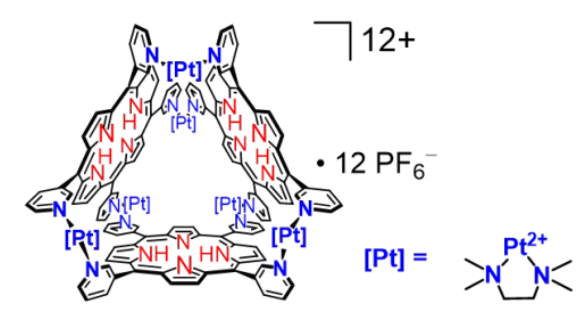

$1 \mathrm{a}$

$10 \%$

[(TMEDA)Pt(NCMe) $)_{2}$ ] 20Tf (3a, $\left.0.459 \mathrm{~g}, 0.664 \mathrm{mmol}\right)$ and (3-py) ${ }_{4}$ porphyrin (2, $0.206 \mathrm{~g}, 0.332$ $\mathrm{mmol}$ ) were combined in $50 \mathrm{~mL}$ MeCN to provide an opaque purple suspension. This mixture was stirred at $100{ }^{\circ} \mathrm{C}$ for $48 \mathrm{~h}$ in a thick-walled $100 \mathrm{~mL}$ Schlenk tube that was sealed with a threaded PTFE stopper (SAFETY NOTE: the sealed Schlenk tube was kept behind a heavy polycarbonate blast shield while the mixture was at elevated temperatures). The resulting dark purple solution was allowed to cool to room temperature before filtering to remove any remaining solids and concentrating the filtrate to about $5 \mathrm{~mL}$ by rotary evaporation. An excess of ammonium hexafluorophosphate $\left(\mathrm{NH}_{4} \mathrm{PF}_{6}, 1 \mathrm{~g} ; 6.1 \mathrm{mmol}\right)$ was added directly to the dark purple solution, followed by $50 \mathrm{~mL}$ of water, resulting in the immediate formation of a dark purple precipitate. The precipitate was collected by vacuum filtration and washed with water $(3 \times 5 \mathrm{~mL})$ to remove 
any remaining $\mathrm{NH}_{4} \mathrm{PF}_{6}$. The ${ }^{1} \mathrm{H}$ NMR spectrum of the crude product in $\mathrm{CD}_{3} \mathrm{CN}$ showed a mixture of ca. $50 \% 1$ a combined with unidentifiable byproducts. A solution of the crude product in $5 \mathrm{~mL}$ DMF was layered with $15 \mathrm{~mL}$ of $\mathrm{Et}_{2} \mathrm{O}$ and stored at $4{ }^{\circ} \mathrm{C}$ for 5 days to provide dark purple crystals of $1 \mathrm{a}$. The supernatant was removed by pipette and the product was washed with additional $\mathrm{Et}_{2} \mathrm{O}$ before drying under active vacuum (12 h at $0.1 \mathrm{Torr}$ ) to provide $71 \mathrm{mg}$ of $1 \mathrm{a}$ as dark purple crystals (10\% yield). ${ }^{1} \mathrm{H}$ NMR $\left(500 \mathrm{MHz}, \mathrm{CD}_{3} \mathrm{CN}\right): \delta 9.56(\mathrm{~d}, J=5.5 \mathrm{~Hz}, 12 \mathrm{H}), 9.26(\mathrm{~s}, 12 \mathrm{H}), 8.61$ (s, broad, $12 \mathrm{H}), 8.58(\mathrm{~d}, \mathrm{~J}=7.8 \mathrm{~Hz}, 12 \mathrm{H}), 8.34(\mathrm{~s}, 12 \mathrm{H}), 8.21(\mathrm{dd}, J=7.8,5.8 \mathrm{~Hz}, 12 \mathrm{H}), 3.37-3.29(\mathrm{~m}, 24 \mathrm{H})$, $3.19(\mathrm{~s}, 36 \mathrm{H}), 3.15(\mathrm{~s}, 36 \mathrm{H}),-4.16(\mathrm{~s}, 6 \mathrm{H}) .{ }^{19} \mathrm{~F} \mathrm{NMR}\left(470 \mathrm{MHz}, \mathrm{CD}_{3} \mathrm{CN}\right): \delta-71.55\left(\mathrm{~d}, J_{F P}=705 \mathrm{~Hz}\right)$. ${ }^{13} \mathrm{C} N M R\left(125 \mathrm{MHz}, \mathrm{CD}_{3} \mathrm{CN}\right): \delta$ 155.6, 152.8, 147.0, 141.9, 128.4, 114.9, 65.6, 53.1, 52.7. ESI(+)MS: $\left[1 \mathbf{a}-4 \mathrm{PF}_{6}{ }^{-}\right]^{4+}$ Calculated: $1220.7363 \mathrm{~m} / \mathrm{z}$, Found: $1220.6835 \mathrm{~m} / \mathrm{z}$; $\left[\mathbf{1 a}-5 \mathrm{PF}_{6}{ }^{-}\right]^{5+}$ Calculated: $947.5962 \mathrm{~m} / \mathrm{z}$, Found: $947.5755 \mathrm{~m} / \mathrm{z} ;\left[\mathbf{1 a}-6 \mathrm{PF}_{6}{ }^{-}\right]^{6+}$ Calculated: $765.6695 \mathrm{~m} / \mathrm{z}$, Found: 765.6698 $\mathrm{m} / \mathrm{z} ;\left[1 \mathrm{a}-7 \mathrm{PF}_{6}{ }^{-}\right]^{7+}$ Calculated: $635.4361 \mathrm{~m} / \mathrm{z}$, Found: $635.4454 \mathrm{~m} / \mathrm{z}$.

*Measured at $2.85 \mathrm{mM}$ 1a. This ${ }^{19} \mathrm{~F}$ chemical shift is concentration dependent. See Figure S17.

B. Cage 1b was prepared by the route depicted in Scheme S2. Procedures for the individual steps are described below.

Scheme S2. Synthesis of $\mathbf{1 b}$.

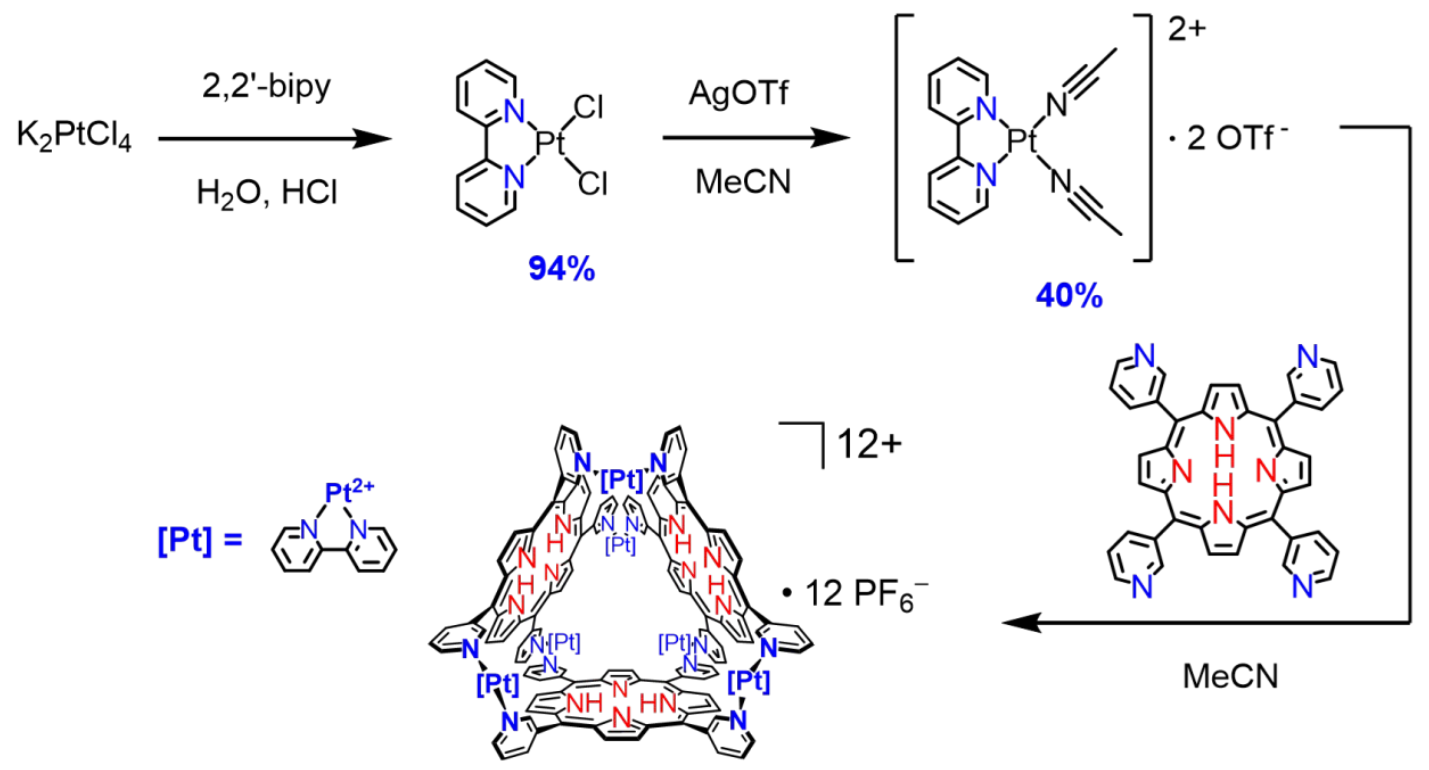

$1 \mathrm{~b}$

$44 \%$ 
(bipy) $\mathrm{PtCl}_{2}$ was synthesized by a modified literature procedure7:
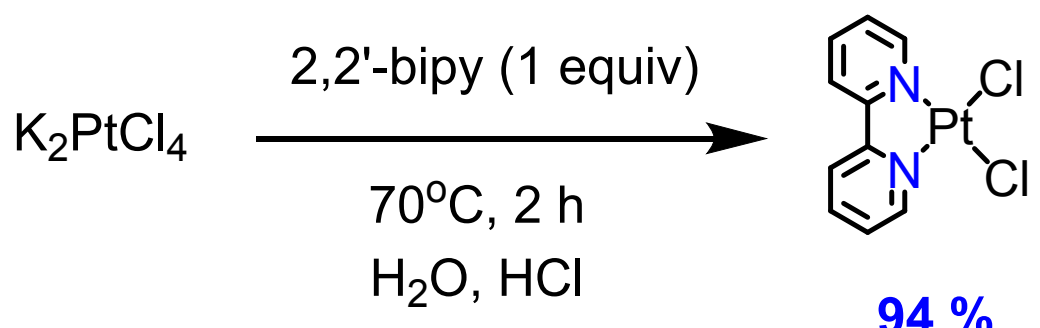

Potassium tetrachloroplatinate $\left(\mathrm{K}_{2} \mathrm{PtCl}_{4}, 0.503 \mathrm{~g}, 1.21 \mathrm{mmol}\right), 2,2^{\prime}$-bipyridine (bipy, $0.189 \mathrm{~g}, 1.21$ $\mathrm{mmol})$, and 2 drops of concentrated hydrochloric acid were added to $40 \mathrm{~mL}$ of water, which was heated to $70{ }^{\circ} \mathrm{C}$ for $2 \mathrm{~h}$. A fluffy yellow precipitate was isolated by vacuum filtration and washed with water, ethanol, and diethyl ether before drying in air to yield $0.487 \mathrm{~g}$ of yellow solid (94\% yield). ${ }^{1} \mathrm{H}$ NMR $\left(500 \mathrm{MHz},\left(\mathrm{CD}_{3}\right)_{2} \mathrm{CO}\right): \delta 9.74(\mathrm{dd}, J=5.8,0.7 \mathrm{~Hz}, 2 \mathrm{H}), 8.52-8.46(\mathrm{~m}, 2 \mathrm{H}), 8.42$ (td, $J=8.1,1.5 \mathrm{~Hz}, 2 \mathrm{H}), 7.83$ (ddd, $J=7.4,5.9,1.5 \mathrm{~Hz}, 2 \mathrm{H}$ ).

[(bipy)Pt(NCMe) $)_{2} \cdot 20 T f(3 b)$ was synthesized by a modified literature procedure ${ }^{8}$ :<smiles></smiles>
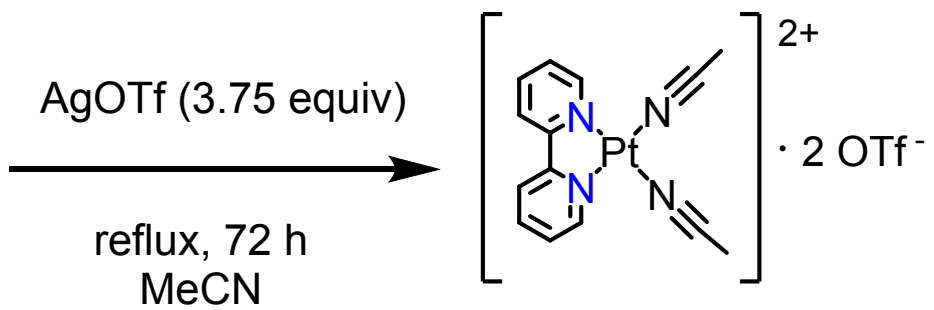

$3 b$

$55 \%$

Silver trifluoromethanesulfonate (AgOTf, $1.11 \mathrm{~g}, 4.32 \mathrm{mmol}$ ) was added to a suspension of (bipy) $\mathrm{PtCl}_{2}(0.487 \mathrm{~g}, 1.15 \mathrm{mmol})$ in $50 \mathrm{~mL}$ of $\mathrm{MeCN}$. The mixture was shielded from light and heated under reflux for 3 days, resulting in the formation of a white precipitate of $\mathrm{AgCl}$. The precipitate was removed using a cellulose filter followed by a $0.2 \mu \mathrm{m}$ PTFE syringe filter. The resulting clear, colorless solution was concentrated using a rotary evaporator until a white precipitate began forming, at which point the solution was placed in a freezer at $-20^{\circ} \mathrm{C}$. After 16 $\mathrm{h}$, off-white crystals of $\mathbf{3 b}$ were collected by vacuum filtration, washed with cold $\mathrm{MeCN}$, and dried under active vacuum ( $12 \mathrm{~h}$ at $0.1 \mathrm{Torr}$ ) to provide $0.402 \mathrm{~g}$ of $\mathbf{3 b}\left(55 \%\right.$ yield). ${ }^{1} \mathrm{H} \mathrm{NMR}(500 \mathrm{MHz}$, $\left.\mathrm{CD}_{3} \mathrm{CN}\right) \delta 8.80(\mathrm{dt}, J=5.8,2.1 \mathrm{~Hz}, 2 \mathrm{H}), 8.47(\mathrm{tt}, J=7.9,1.4 \mathrm{~Hz}, 2 \mathrm{H}), 8.37(\mathrm{dt}, J=8.3,2.0 \mathrm{~Hz}, 2 \mathrm{H})$, $7.82(\mathrm{dtt}, J=7.5,3.5,1.6 \mathrm{~Hz}, 2 \mathrm{H})$. 


\section{Cage 1b.}

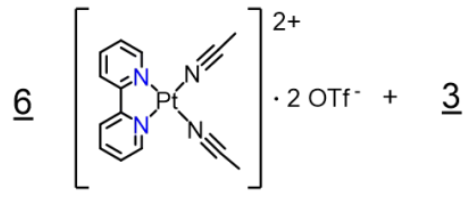

3b

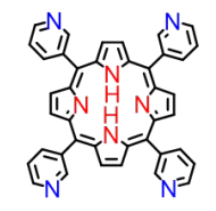

2

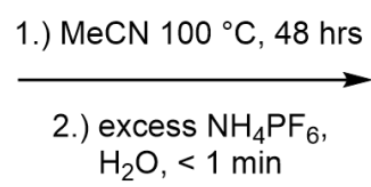
1.) $\mathrm{MeCN} 100^{\circ} \mathrm{C}, 48 \mathrm{hrs}$
$\mathrm{H}_{2} \mathrm{O},<1 \mathrm{~min}$

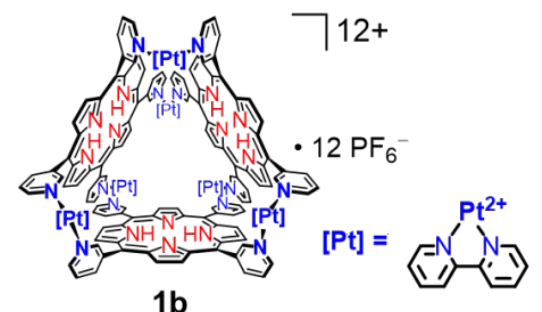

1b

$44 \%$

[(bipy)Pt(NCMe) $)_{2}$ ·2OTf (3b, $0.312 \mathrm{~g}, 0.427 \mathrm{mmol}$ ) and (3-py) ${ }_{4}$ porphyrin (2, $\left.0.132 \mathrm{~g}, 0.213 \mathrm{mmol}\right)$ were combined with $50 \mathrm{~mL}$ MeCN in a thick-walled $100 \mathrm{~mL}$ Schlenk tube. The tube was sealed with a threaded PTFE stopper and the opaque purple suspension was stirred while heating at 100 ${ }^{\circ} \mathrm{C}$ for $48 \mathrm{~h}$ (SAFETY NOTE: the sealed Schlenk tube was kept behind a heavy polycarbonate blast shield while the mixture was at elevated temperatures). The resulting dark purple solution was allowed to cool to room temperature before filtering to remove any remaining solids and concentrating to about $5 \mathrm{~mL}$ using a rotary evaporator. An excess of ammonium hexafluorophosphate $\left(\mathrm{NH}_{4} \mathrm{PF}_{6}, 0.830 \mathrm{~g} ; 5.03 \mathrm{mmol}\right.$ ) was added directly to the solution, followed by $50 \mathrm{~mL}$ of water, resulting in the immediate formation of a dark purple precipitate. The precipitate was collected by vacuum filtration and washed with water $(3 \times 5 \mathrm{~mL})$ to remove $\mathrm{NH}_{4} \mathrm{PF}_{6}$. The ${ }^{1} \mathrm{H}$ NMR spectrum of the crude product in $\mathrm{CD}_{3} \mathrm{CN}$ showed a mixture of ca. $50 \% \mathbf{1 b}$ combined with unidentifiable byproducts. $\mathrm{Et}_{2} \mathrm{O}$ vapors were allowed to diffuse into a solution of the crude product in $\mathrm{MeCN}(5 \mathrm{~mL})$ at $4{ }^{\circ} \mathrm{C}$ for 5 days to provide dark purple crystals of $\mathbf{1 b}$. Pure $\mathbf{1 b}$ was isolated after removing the supernatant, washing the crystals with additional $\mathrm{Et}_{2} \mathrm{O}$, and drying under active vacuum for $12 \mathrm{~h}$ at 0.1 Torr $\left(0.170 \mathrm{~g}, 44 \%\right.$ yield). ${ }^{1} \mathrm{H} \mathrm{NMR}\left(500 \mathrm{MHz}, \mathrm{CD}_{3} \mathrm{CN}\right)$ : $\delta 9.68(\mathrm{~d}, \mathrm{~J}=5.2 \mathrm{~Hz}, 12 \mathrm{H}), 9.41(\mathrm{~s}, 12 \mathrm{H}), 8.71-8.47(\mathrm{~m}, 60 \mathrm{H}), 8.31-8.26(\mathrm{dd}, J=7.7,5.8 \mathrm{~Hz}, 12 \mathrm{H})$, $8.13(\mathrm{~d}, \mathrm{~J}=5.3 \mathrm{~Hz}, 12 \mathrm{H}), 7.93(\mathrm{ddd}, J=7.2,5.8,1.4 \mathrm{~Hz}, 12 \mathrm{H}),-4.23(\mathrm{~s}, 5 \mathrm{H}) .{ }^{19} \mathrm{~F} \mathrm{NMR}(470 \mathrm{MHz}$, $\left.\mathrm{CD}_{3} \mathrm{CN}\right) \delta-71.77\left(\mathrm{~d}, \mathrm{~J}_{F P}=708 \mathrm{~Hz}\right) .{ }^{13} \mathrm{C}$ NMR $\left(125 \mathrm{MHz}, \mathrm{CD}_{3} \mathrm{CN}\right): \delta 157.7,154.5,153.4,150.5$, 148.2, 144.0, 142.3, 129.7, 128.8, 126.3, 114.8. ESI(+)-MS: [1b - 4PF ${ }_{6}{ }^{4+}$ Calculated: 1280.8924 $\mathrm{m} / \mathrm{z}$, Found: $1280.8354 \mathrm{~m} / \mathrm{z}$; $\left[\mathbf{1 b}-5 \mathrm{PF}_{6}{ }^{-}\right]^{5+}$ Calculated: $995.7211 \mathrm{~m} / \mathrm{z}$, Found: $995.7007 \mathrm{~m} / \mathrm{z}$; [1b $\left.-6 \mathrm{PF}_{6}{ }^{-}\right]^{6+}$ Calculated: $805.6069 \mathrm{~m} / \mathrm{z}$, Found: $805.6053 \mathrm{~m} / \mathrm{z}$; [1b - 7PF $\left.{ }_{6}^{-}\right]^{7+}$ Calculated: 669.6681 $\mathrm{m} / \mathrm{z}$, Found: $669.6811 \mathrm{~m} / \mathrm{z}$.

*Measured at $2.85 \mathrm{mM} \mathbf{1 b}$. This ${ }^{19} \mathrm{~F}$ chemical shift is concentration dependent. See Figure S20.

$\mathbf{1 b} \cdot \mathbf{1 2} \mathrm{BF}_{4}$. The $\mathrm{BF}_{4}{ }^{-}$salt of $\mathbf{1 b}$ was prepared by first converting the $\mathrm{PF}_{6}{ }^{-}$salt to the $\mathrm{HSO}_{4}{ }^{-}$salt via metathesis with tetrabutylammonium hydrogen sulfate in $\mathrm{MeCN}$. The resulting precipitate was dissolved in water, and the $\mathrm{BF}_{4}{ }^{-}$salt of $\mathbf{1 b}$ was precipitated upon addition of excess ammonium tetrafluoroborate, providing $\mathbf{1 b} \cdot \mathbf{1 2 B F}_{4}$ in quantitative yield. ${ }^{1} \mathrm{H} N \mathrm{NMR}\left(500 \mathrm{MHz}, \mathrm{CD}_{3} \mathrm{CN}\right): \delta 9.70(\mathrm{~d}$, $J=5.4 \mathrm{~Hz}, 12 \mathrm{H}), 9.44(\mathrm{~s}, 12 \mathrm{H}), 8.72-8.49(\mathrm{~m}, 60 \mathrm{H}), 8.28(\mathrm{dd}, J=7.8,5.8 \mathrm{~Hz}, 12 \mathrm{H}), 8.18(\mathrm{~d}, J=5.5$ $\mathrm{Hz}, 12 \mathrm{H}), 7.93(\mathrm{td}, J=5.8,3.0 \mathrm{~Hz}, 12 \mathrm{H}),-4.24(\mathrm{~s}, 6 \mathrm{H})$. 


\section{C. [(TMEDA)Pt(py) 2$] \cdot 20 T f(5 a)$}

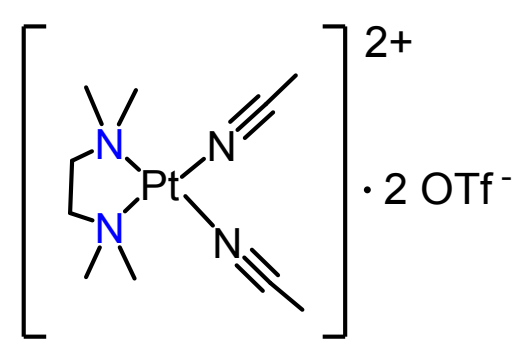

3a

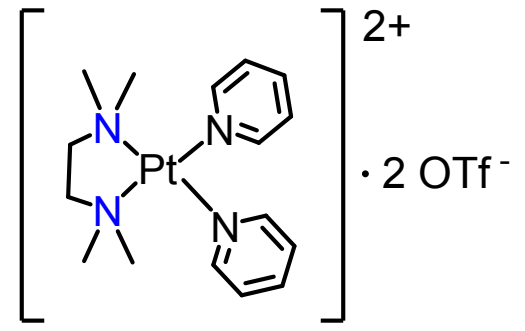

$5 a$

\section{$16 \%$}

[(TMEDA)Pt(NCMe) $)_{2}$-2OTf (3a, $\left.80.4 \mathrm{mg}, 0.116 \mathrm{mmol}\right)$ and pyridine $(28.2 \mathrm{mg}, 0.357 \mathrm{mmol}$ ) were dissolved in $10 \mathrm{~mL}$ of acetonitrile to provide a clear, colorless solution that was heated under reflux for 8 hours. The resulting light-yellow solution was evaporated using a rotary evaporator to provide a beige oil that crystallized upon standing. The product was extracted with $5 \mathrm{~mL}$ of dichloromethane to give a light-yellow solution that was passed through a $0.22 \mu \mathrm{m}$ syringe filter to provide a colorless solution that was evaporated with a gentle stream of $\mathrm{N}_{2}$. The resulting crystalline product was sonicated for 2 min under $1 \mathrm{~mL}$ of dichloromethane, followed by removing the wash solution by pipette and drying the remaining powder under active vacuum ( 0.1 Torr) for $8 \mathrm{~h}$ to provide pure $1 \mathrm{a}$ as a fine white powder ( $14.4 \mathrm{mg}, 16 \%$ yield). ${ }^{1} \mathrm{H}$ NMR (500 $\mathrm{MHz}, \mathrm{CD}_{3} \mathrm{CN}$ ): $\delta 8.87(\mathrm{dd}, J=5.7,1.4 \mathrm{~Hz}, J(\mathrm{Pt}-\mathrm{H})=34 \mathrm{~Hz}, 4 \mathrm{H}), 7.97(\mathrm{tt}, J=7.8,1.4 \mathrm{~Hz}, 2 \mathrm{H}), 7.57$ (dd, J = 7.8, $5.7 \mathrm{~Hz}, 4 \mathrm{H}), 2.98(\mathrm{~s}, J(\mathrm{Pt}-\mathrm{H})=22 \mathrm{~Hz}, 4 \mathrm{H}), 2.69(\mathrm{~s}, J(\mathrm{Pt}-\mathrm{H})=27 \mathrm{~Hz}, 12 \mathrm{H}) .{ }^{13} \mathrm{C}$ NMR $(125$ $\left.\mathrm{MHz}, \mathrm{CD}_{3} \mathrm{CN}\right): \delta 153.39,142.42,129.48,65.66,52.79$. ESI(+)-MS: [(TMEDA)Pt(Py) $\left.)_{2} \cdot \mathrm{OTf}^{-}\right]^{+}$ Calculated: $618.1277 \mathrm{~m} / \mathrm{z}$, Found: $618.1264 \mathrm{~m} / \mathrm{z}$. 


\section{1D and 2D NMR Characterization}

Nuclear magnetic resonance (NMR) spectra were measured using a Bruker AVANCE Neo spectrometer or a Varian VNMR spectrometer, both with a $500 \mathrm{MHz}$ working frequency for ${ }^{1} \mathrm{H}$ nuclei, $470 \mathrm{MHz}$ for ${ }^{19} \mathrm{~F}$ nuclei, and $125 \mathrm{MHz}$ for ${ }^{13} \mathrm{C}$ nuclei. ${ }^{1} \mathrm{H}$ NMR spectra were referenced to the residual proteo signal of the solvent $\left({ }^{1} \mathrm{H} \delta 1.94 \mathrm{ppm}\right.$ for $\mathrm{CD}_{2} \mathrm{HCN}$ in $\mathrm{CD}_{3} \mathrm{CN}, 2.05 \mathrm{ppm}$ for $\left.\left(\mathrm{CD}_{2} \mathrm{H}\right)\left(\mathrm{CD}_{3}\right) \mathrm{CO}\right) ;{ }^{13} \mathrm{C}\left\{{ }^{1} \mathrm{H}\right\}$ NMR spectra were referenced to the solvent ${ }^{13} \mathrm{C}\left\{{ }^{1} \mathrm{H}\right\}$ signal $\left({ }^{13} \mathrm{C} \delta 118.26 \mathrm{ppm}\right.$ for $\left.\mathrm{CD}_{3} \underline{\mathrm{CN}}\right) \cdot{ }^{19} \mathrm{~F}$ NMR spectra were referenced to a capillary standard of fluorobenzene in $\mathrm{CD}_{3} \mathrm{CN}\left({ }^{19} \mathrm{~F} \delta-114.26 \mathrm{ppm}\right)$. The signal of fluorobenzene in $\mathrm{CD}_{3} \mathrm{CN}$ was calibrated against a capillary standard of neat fluorobenzene $\left({ }^{19} \mathrm{~F} \delta-113.5\right.$ ppm relative to $\mathrm{CFCl}_{3}$ @ $0 \mathrm{ppm}){ }^{10} \cdot{ }^{13} \mathrm{C}\left\{{ }^{1} \mathrm{H}\right\}$ NMR spectra were acquired with 10,000 scans and a $10 \mathrm{~s}$ relaxation delay. Resonances for the porphyrin quaternary carbons were not observed for $\mathbf{1 a , 1} \mathbf{1 b}$.

\section{Cage 1a}

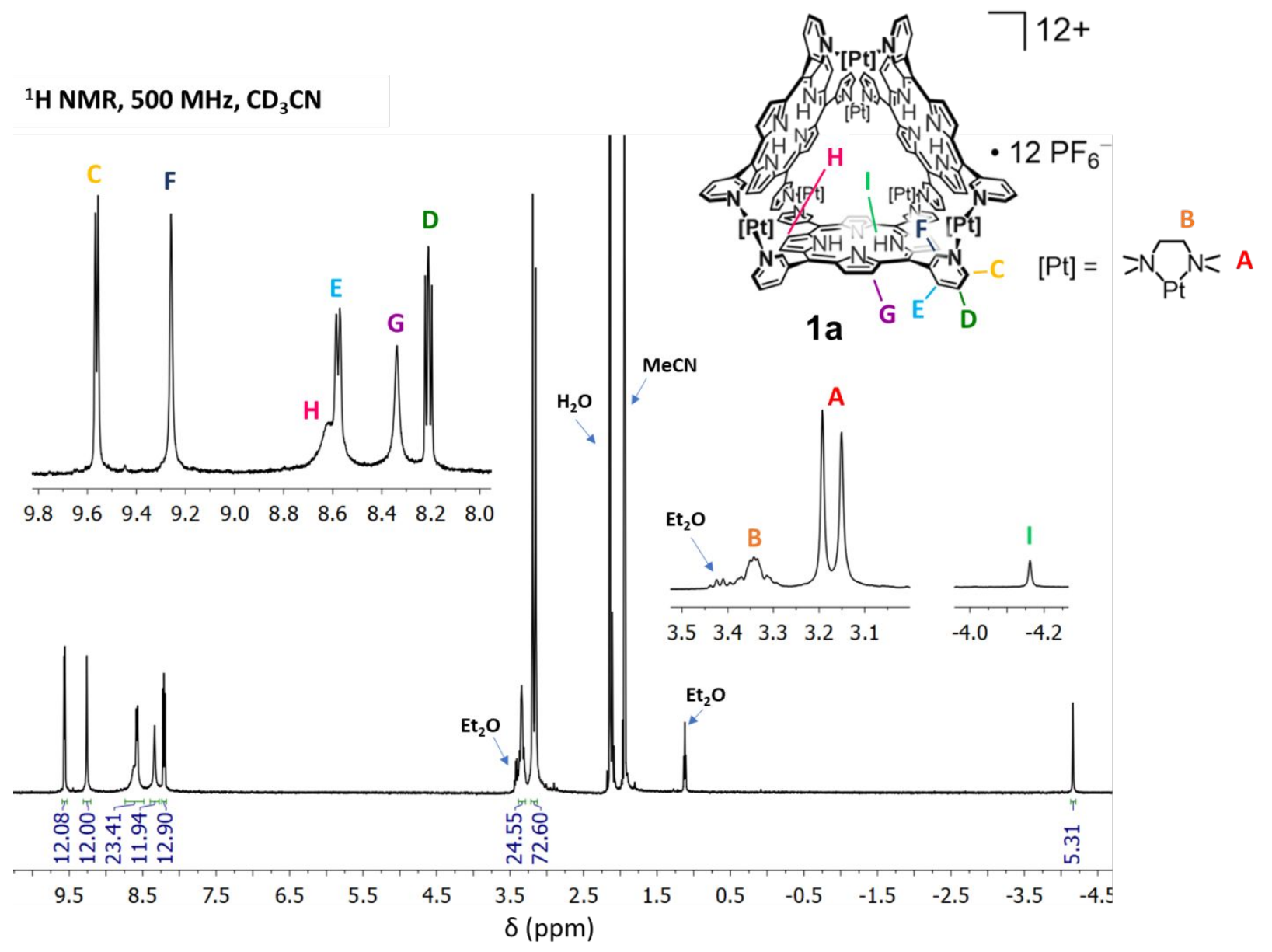

Figure S1: ${ }^{1} \mathrm{H} N M R$ spectrum of $1 \mathrm{a}$ in $\mathrm{CD}_{3} \mathrm{CN}$. The resonances $\mathrm{G}$ and $\mathrm{H}$ were assigned based on the greater influence of anion binding on resonance $\mathrm{G}$ (see Figure S18), which is therefore attributed to the pyrrolic $\mathrm{CH}$ position that is closer to the internally bound $\mathrm{PF}_{6}{ }^{-}$anions in the solid state structure of 1a (see Figure S16). 
${ }^{13} \mathrm{C}\left\{{ }^{1} \mathrm{H}\right\} \mathrm{NMR}, 125 \mathrm{MHz}, \mathrm{CD}_{3} \mathrm{CN}$
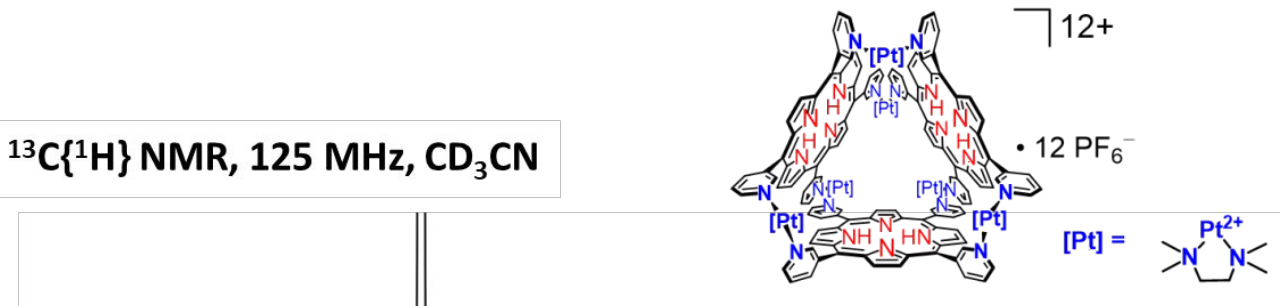

$1 a$
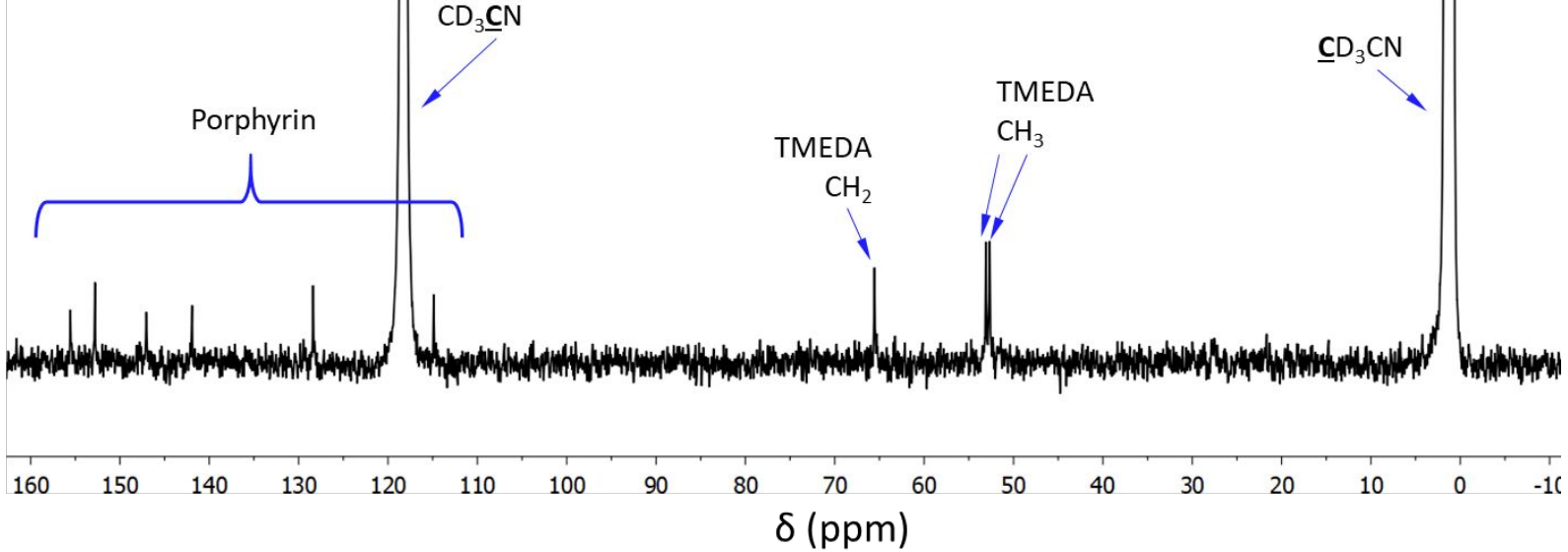

Figure S2: ${ }^{13} \mathrm{C}\left\{{ }^{1} \mathrm{H}\right\}$ NMR spectrum of $1 \mathrm{a}$ in $\mathrm{CD}_{3} \mathrm{CN}$. Note that resonances for quaternary carbons were not observed.

${ }^{19} \mathrm{~F} N \mathrm{NMR}, 470 \mathrm{MHz}, \mathrm{CD}_{3} \mathrm{CN}$

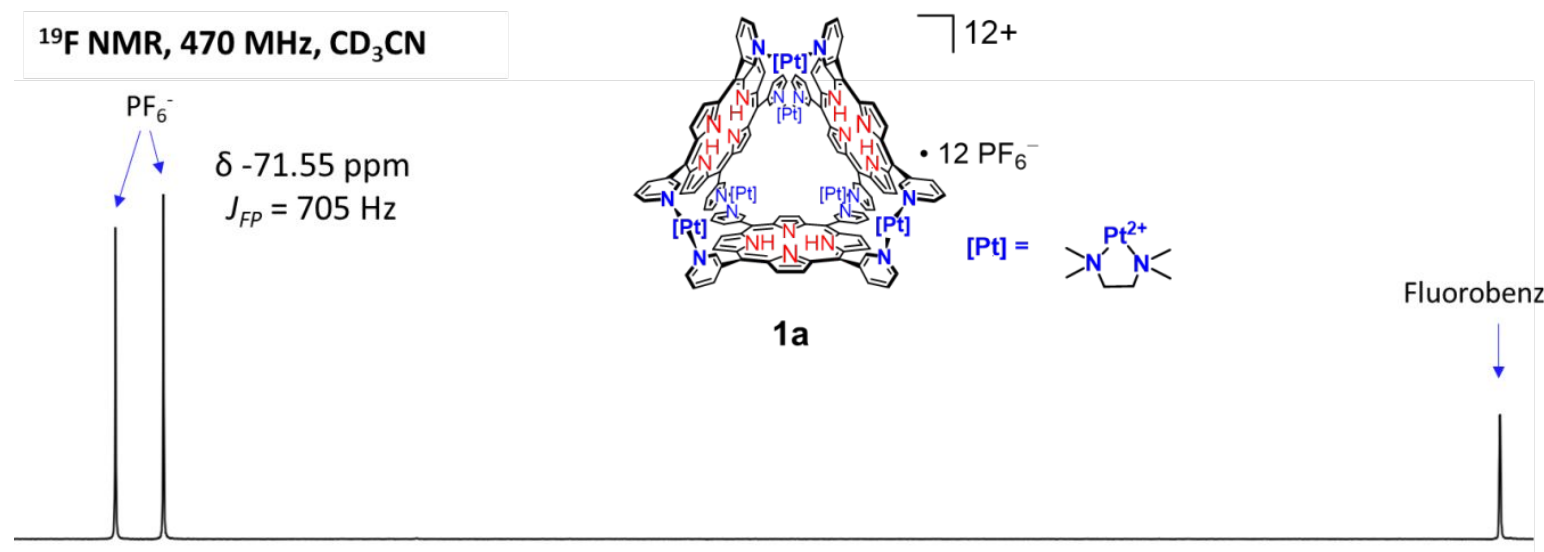

1a

Fluorobenzene

$\begin{array}{rlllllllllllllllllllllll}-68 & -70 & -72 & -74 & -76 & -78 & -80 & -82 & -84 & -86 & -88 & -90 & -92 & -94 & -96 & -98 & -100 & -102 & -104 & -106 & -108 & -110 & -112 & -114\end{array}$

Figure S3: ${ }^{19} \mathrm{~F}$ NMR spectrum of the $\mathrm{PF}_{6}{ }^{-}$anions in a $2.85 \mathrm{mM}$ sample of $1 \mathrm{a}$ in $\mathrm{CD}_{3} \mathrm{CN}$. 


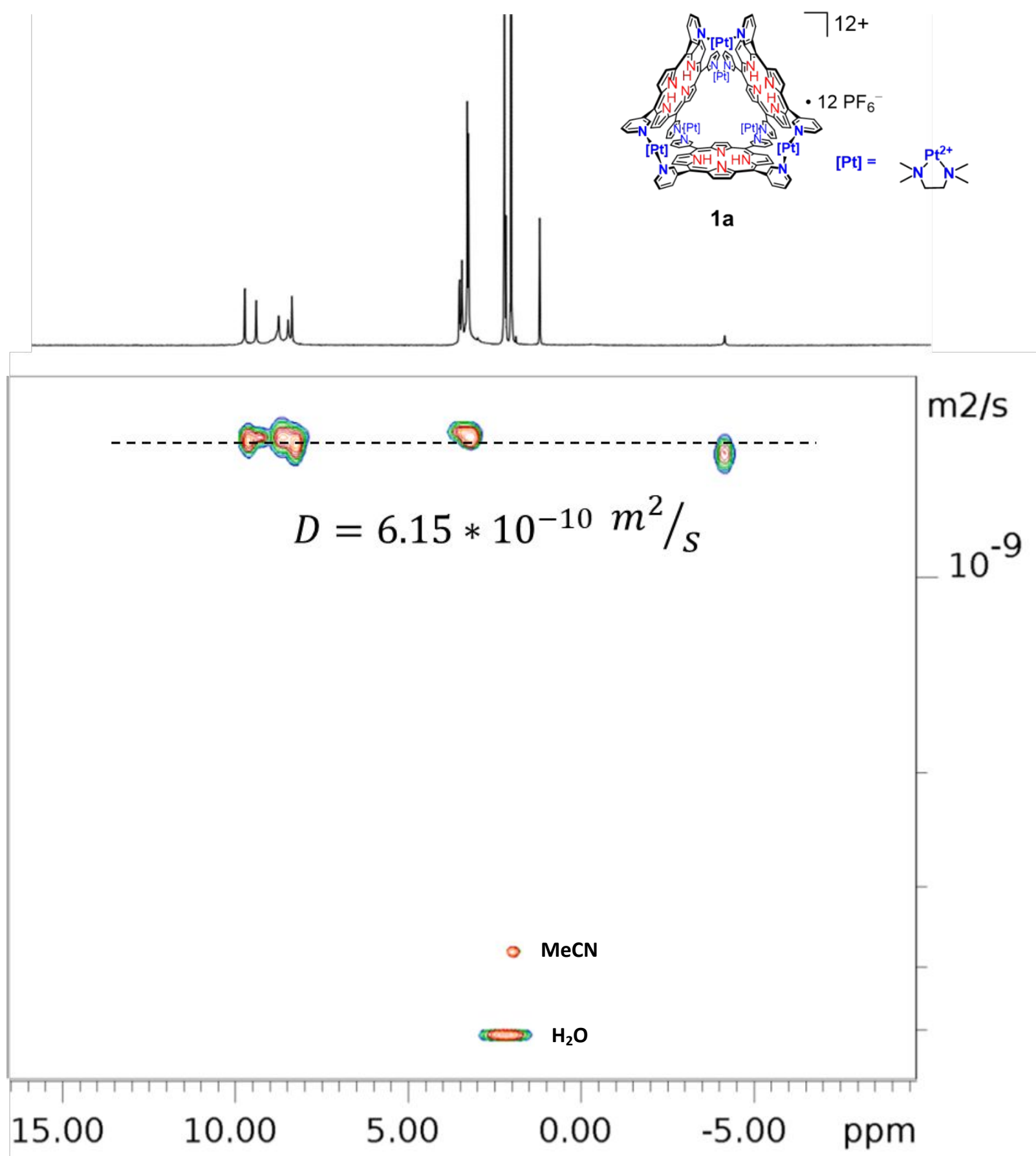

Figure S4: Diffusion ordered (DOSY) ${ }^{1} \mathrm{H}$ NMR spectrum of $1 \mathrm{a}$ in $\mathrm{CD}_{3} \mathrm{CN}$. The experimental diffusion constant corresponds to a spherical hydrodynamic radius ${ }^{11}$ of $10.5 \AA$. This radius is consistent with the solid-state structure of $1 \mathrm{a}$, which shows a length of 18.45(5) A between the outermost carbon atoms of the linkers (corresponding to a length of ca. $21.5 \mathrm{~A}$ between the outermost van der Waals surfaces). 


\section{Cage 1b}

${ }^{1} \mathrm{H}$ NMR, $500 \mathrm{MHz}, \mathrm{CD}_{3} \mathrm{CN}$

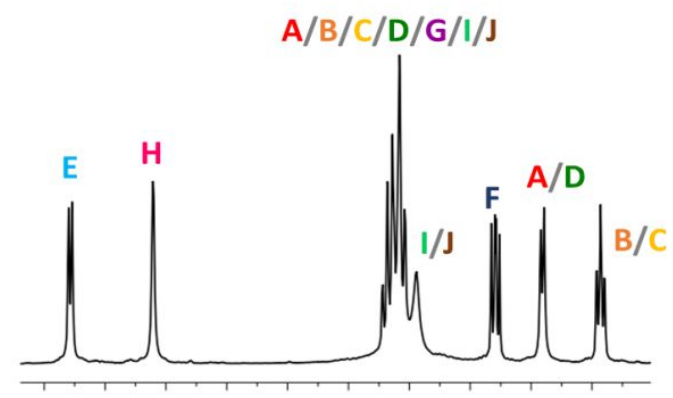

$\begin{array}{llllllllll}9.8 & 9.6 & 9.4 & 9.2 & 9.0 & 8.8 & 8.6 & 8.4 & 8.2 & 8.0\end{array}$
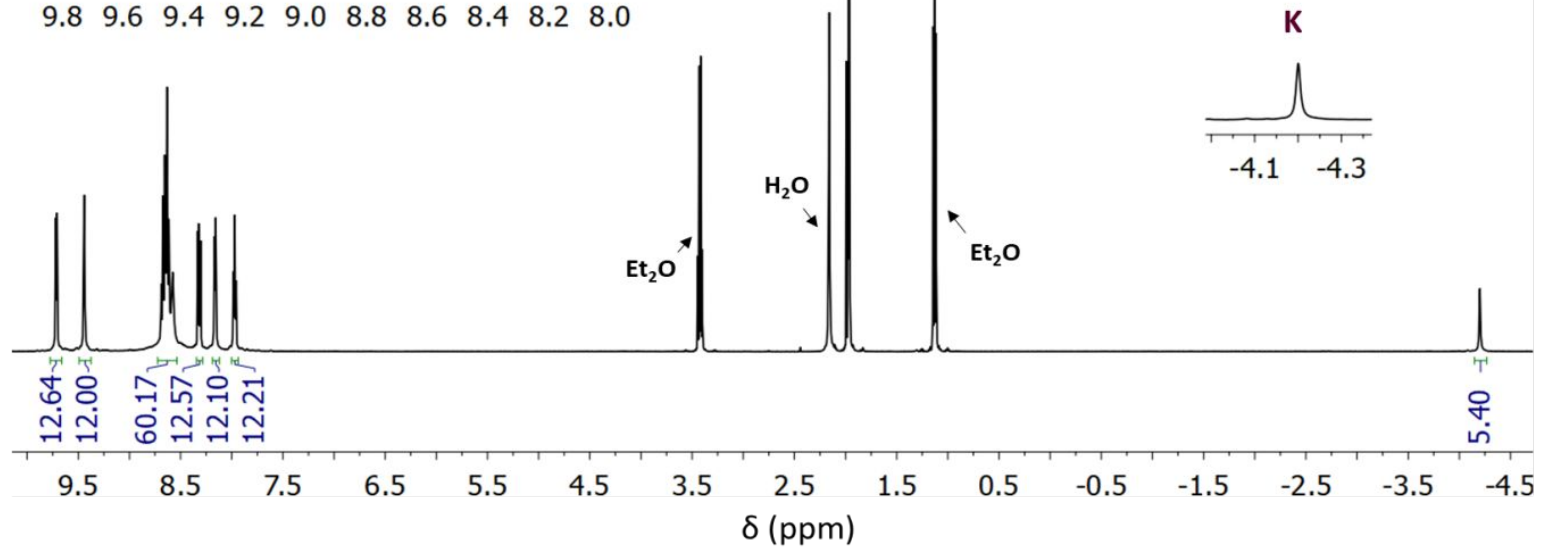

Figure S5: ${ }^{1} \mathrm{H}$ NMR spectrum of $\mathbf{1 b}$ in $\mathrm{CD}_{3} \mathrm{CN}$. Resonance $\mathrm{F}$ was assigned based on the ${ }^{1} \mathrm{H}-{ }^{1} \mathrm{H}$ COSY spectrum (see Figure S8). Certain resonances could not be assigned definitively.

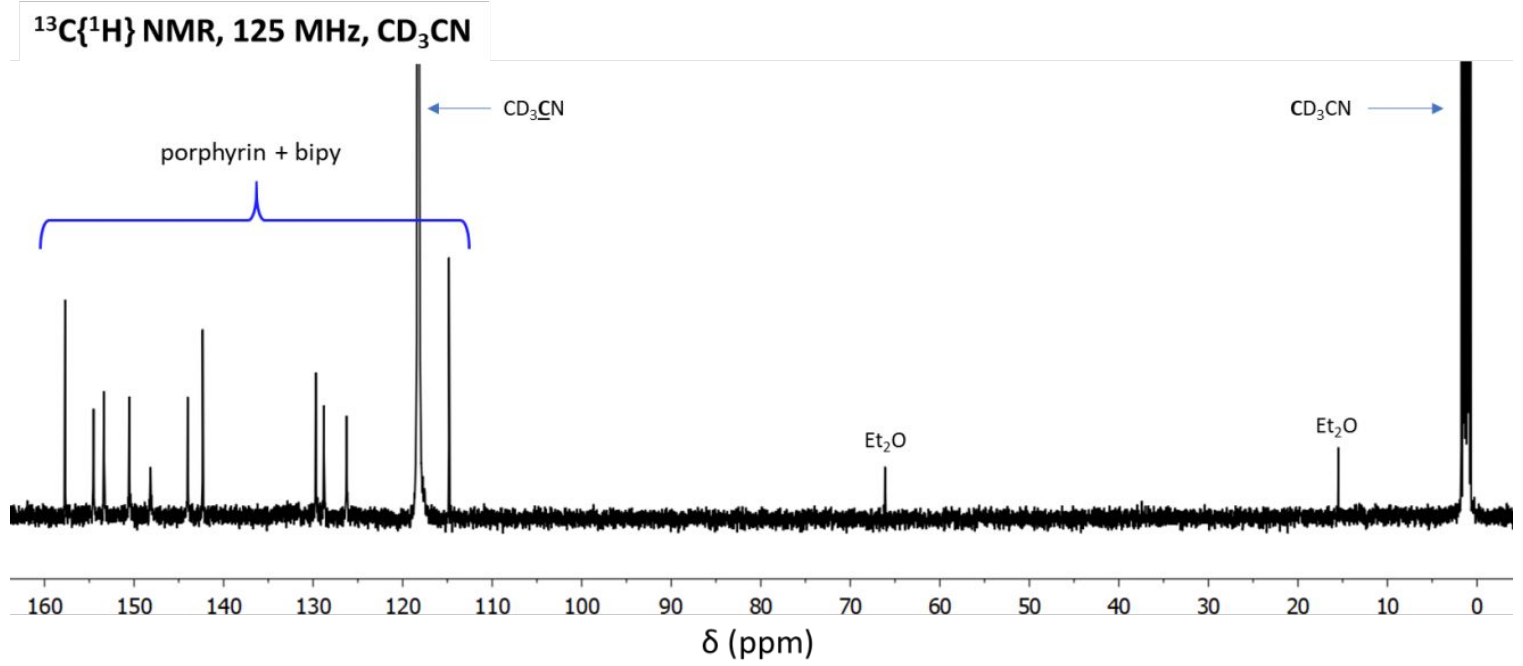

Figure S6: ${ }^{13} \mathrm{C}\left\{{ }^{1} \mathrm{H}\right\}$ NMR spectrum of $\mathbf{1 b}$ in $\mathrm{CD}_{3} \mathrm{CN}$. Note that resonances for the porphyrin quaternary carbons were not observed. 


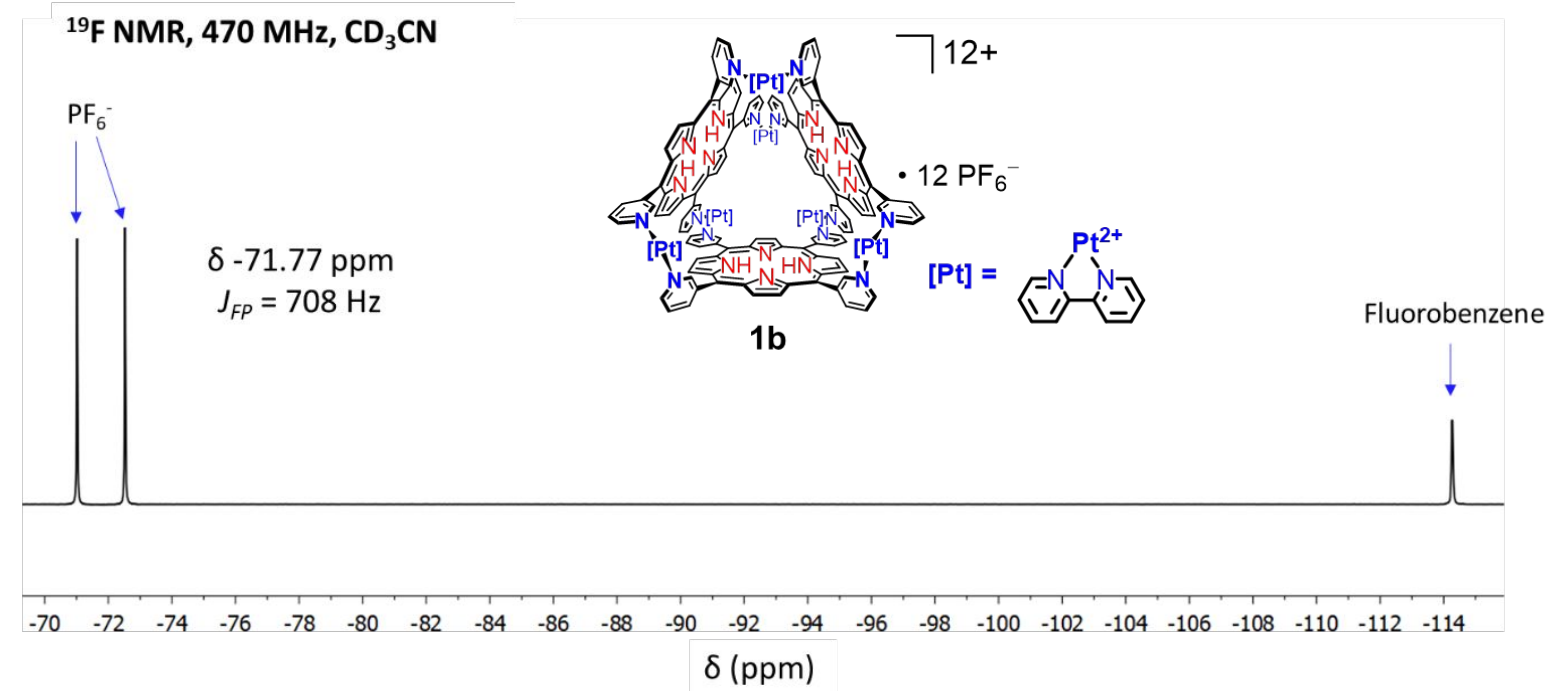

Figure S7: ${ }^{19} \mathrm{~F}$ NMR spectrum of a $2.85 \mathrm{mM}$ solution of $\mathbf{1 b}$ in $\mathrm{CD}_{3} \mathrm{CN}$.

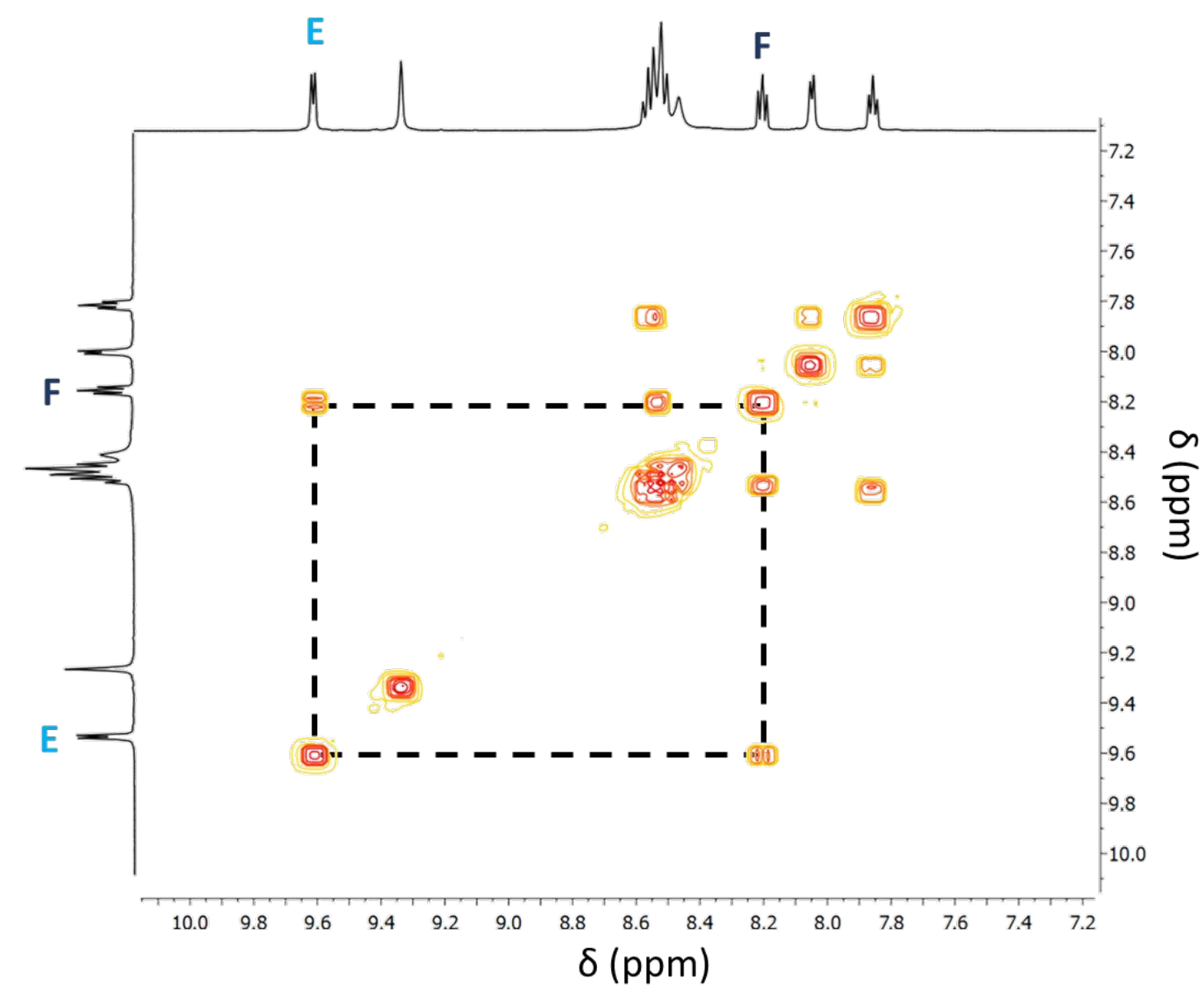

Figure S8: ${ }^{1} \mathrm{H}-{ }^{1} \mathrm{H}$ correlation (COSY) spectrum of $\mathbf{1 b}$. Only the aromatic cross-region is shown. Cross peaks used to distinguish $\mathrm{F}$ as belonging to the 3-py groups are marked with dotted lines. 


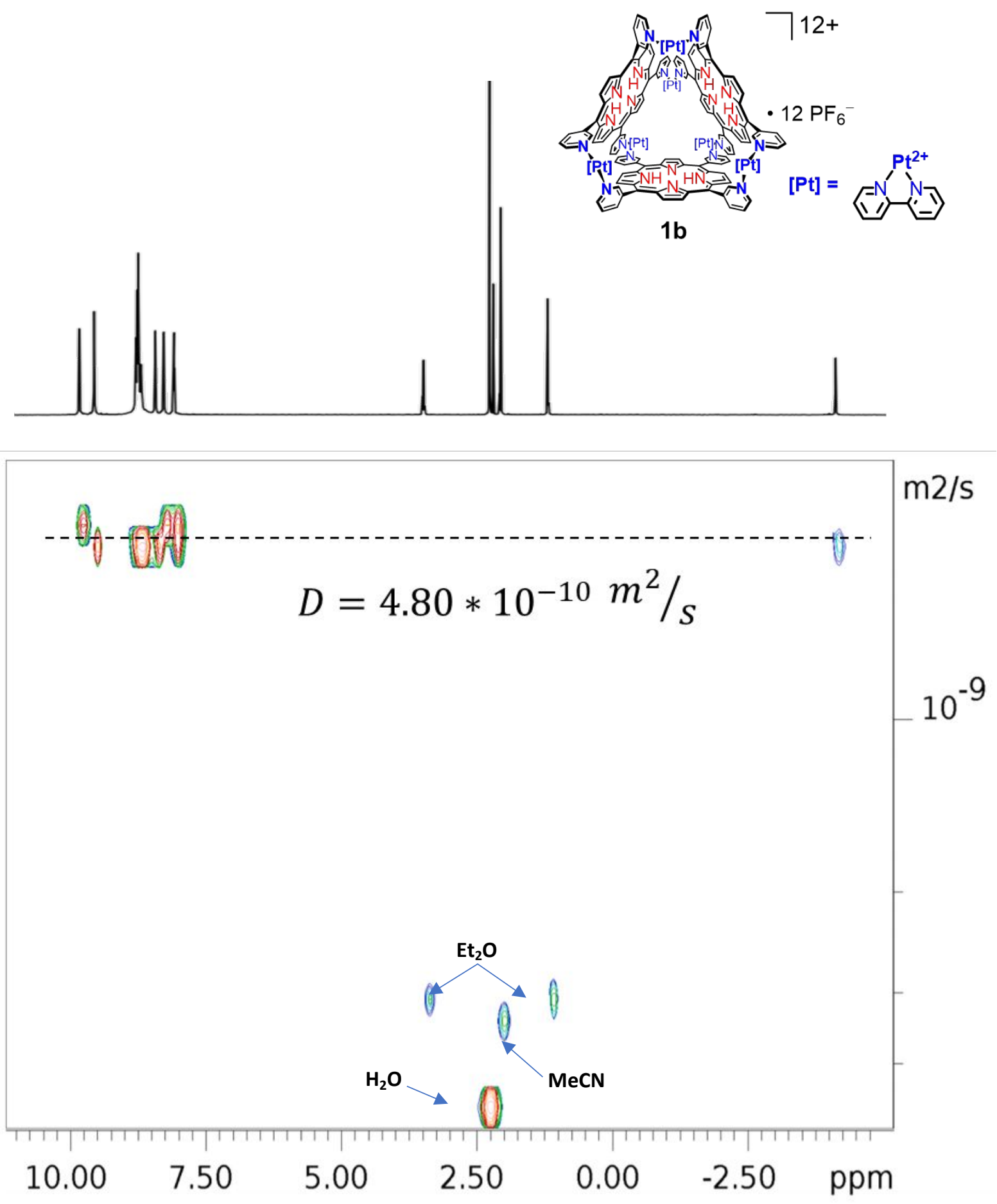

Figure S9: Diffusion ordered (DOSY) ${ }^{1} \mathrm{H}$ NMR spectrum of $\mathbf{1 b}$ in $\mathrm{CD}_{3} \mathrm{CN}$. The experimental diffusion constant corresponds to a spherical hydrodynamic radius ${ }^{11}$ of $13.4 \AA$. This radius is slightly larger than that measured for $1 \mathrm{a}(10.5 \AA$, Figure S4), consistent with the slightly larger size expected for 1b based on reported structures of the Pd-based versions of both cages. ${ }^{5}$ 


\section{ESI(+)-MS Characterization}

\section{Cage 1a}

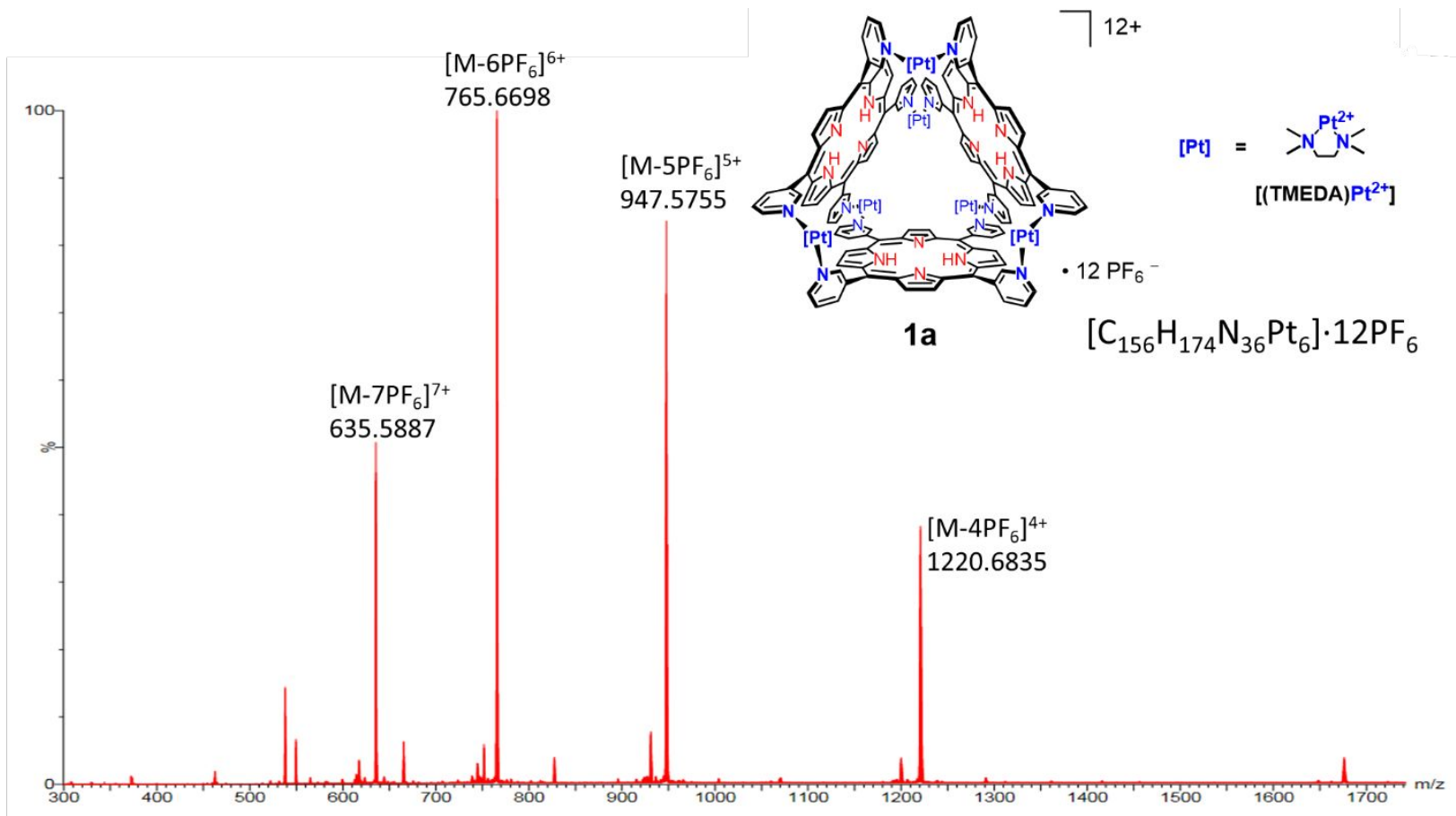

Figure S10: $\mathrm{ESI}(+)-\mathrm{MS}$ spectrum of $1 \mathrm{a}$ in $\mathrm{CH}_{3} \mathrm{CN}$, showing successive loss of $\mathrm{PF}_{6}{ }^{-}$from $1 \mathrm{a}$.
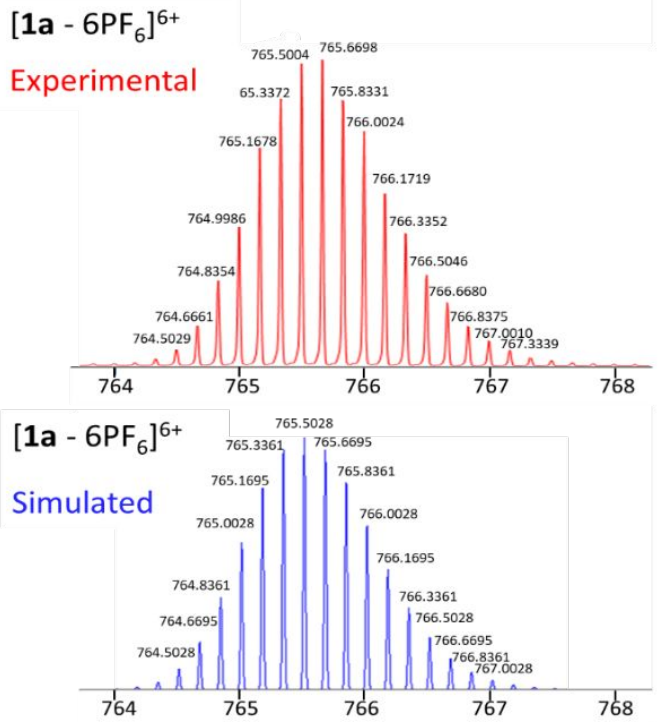

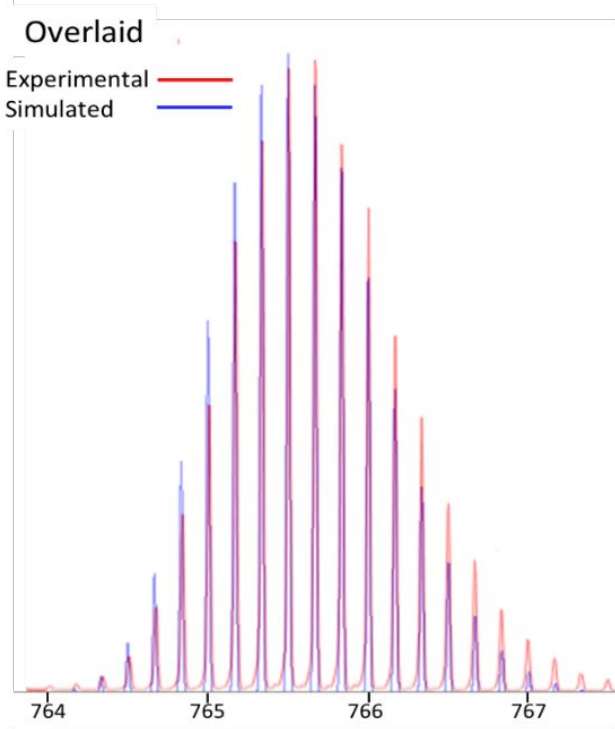

Figure S11: $\mathrm{ESI}(+)-\mathrm{MS}$ spectrum of $1 \mathrm{a}$ in $\mathrm{CH}_{3} \mathrm{CN}$. Experimental (red) and simulated (blue) spectra of $\left[1 \mathrm{a}-6 \mathrm{PF}_{6}\right]^{6+}$ showing isotopic distributions. An overlay of the experimental and simulated isotope pattern shows good agreement. 


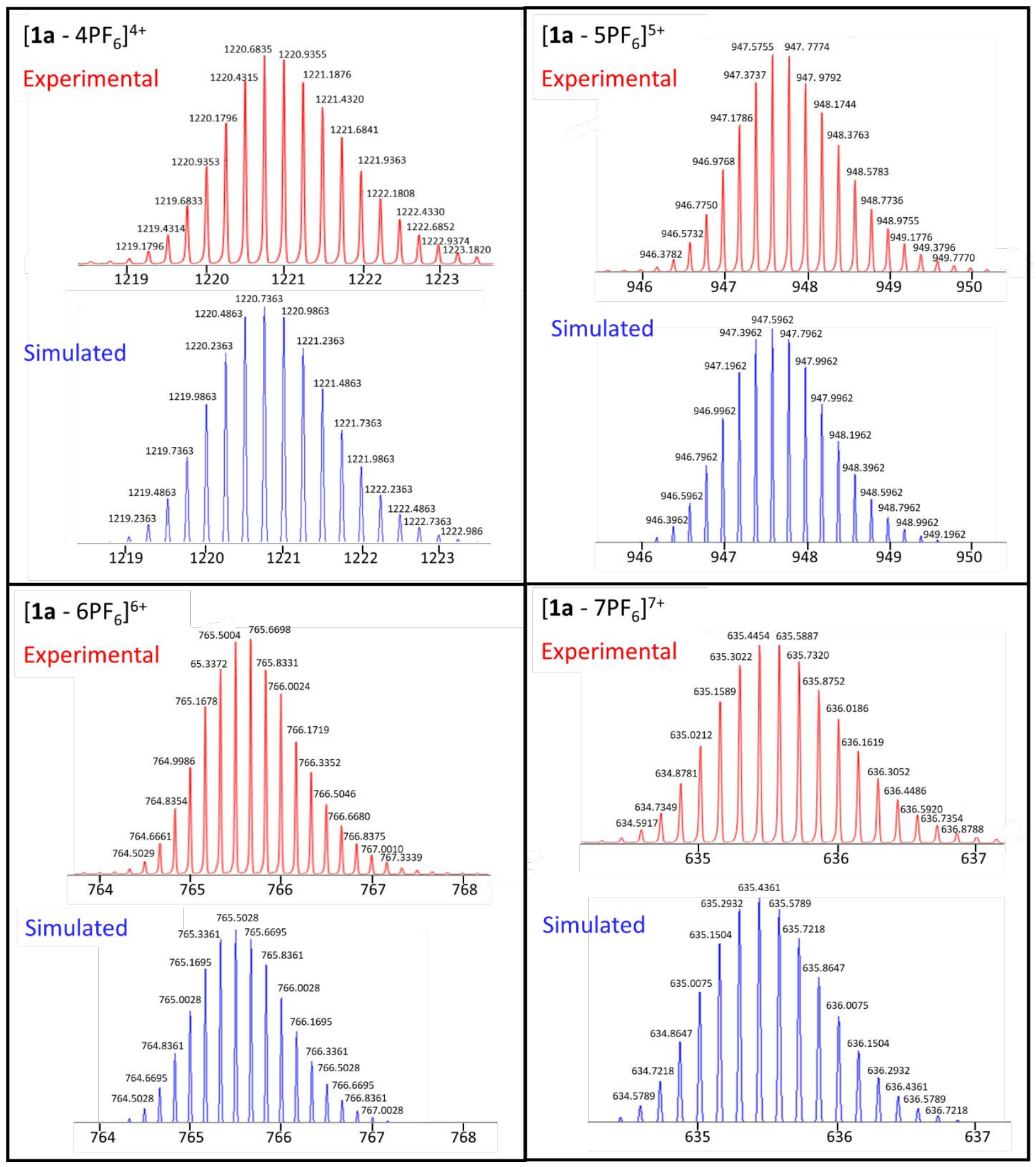

Figure S12: ESI(+)-MS peaks corresponding to the loss of 4 to $7 \mathrm{PF}_{6}{ }^{-}$anions from 1a. Experimental (red) and simulated (blue) peaks are presented for $\left[1 \mathrm{a}-4 \mathrm{PF}_{6}\right]^{4+},\left[1 \mathrm{a}-5 \mathrm{PF}_{6}\right]^{5+},\left[1 \mathrm{a}-6 \mathrm{PF}_{6}\right]^{6+}$, and $\left[1 \mathrm{a}-7 \mathrm{PF} \mathrm{F}^{7+}\right.$. 


\section{Cage 1b}

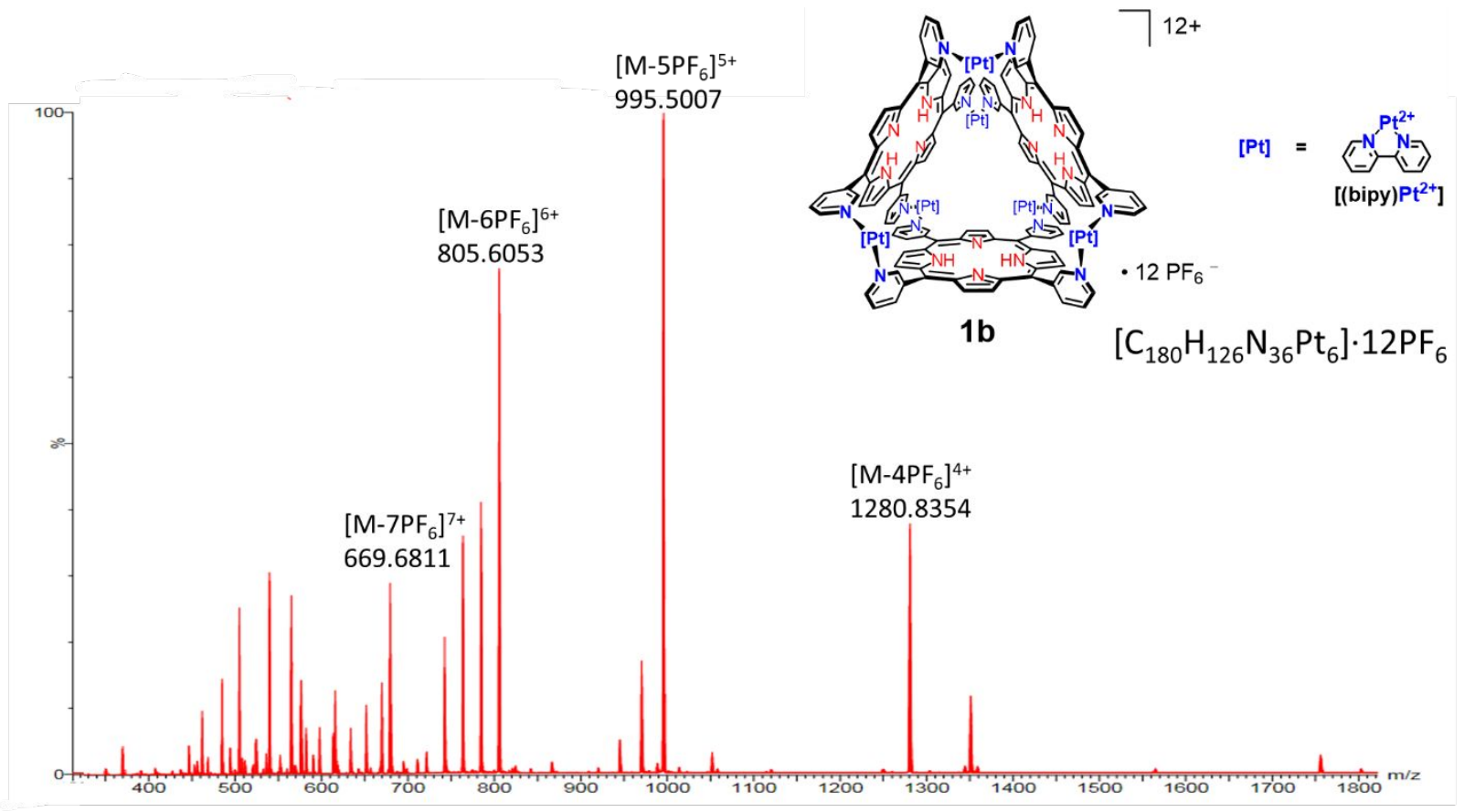

Figure S13: $\mathrm{ESI}(+)-\mathrm{MS}$ spectrum of $\mathbf{1 b}$ in $\mathrm{CH}_{3} \mathrm{CN}$, showing successive loss of $\mathrm{PF}_{6}{ }^{-}$from $\mathbf{1 b}$.
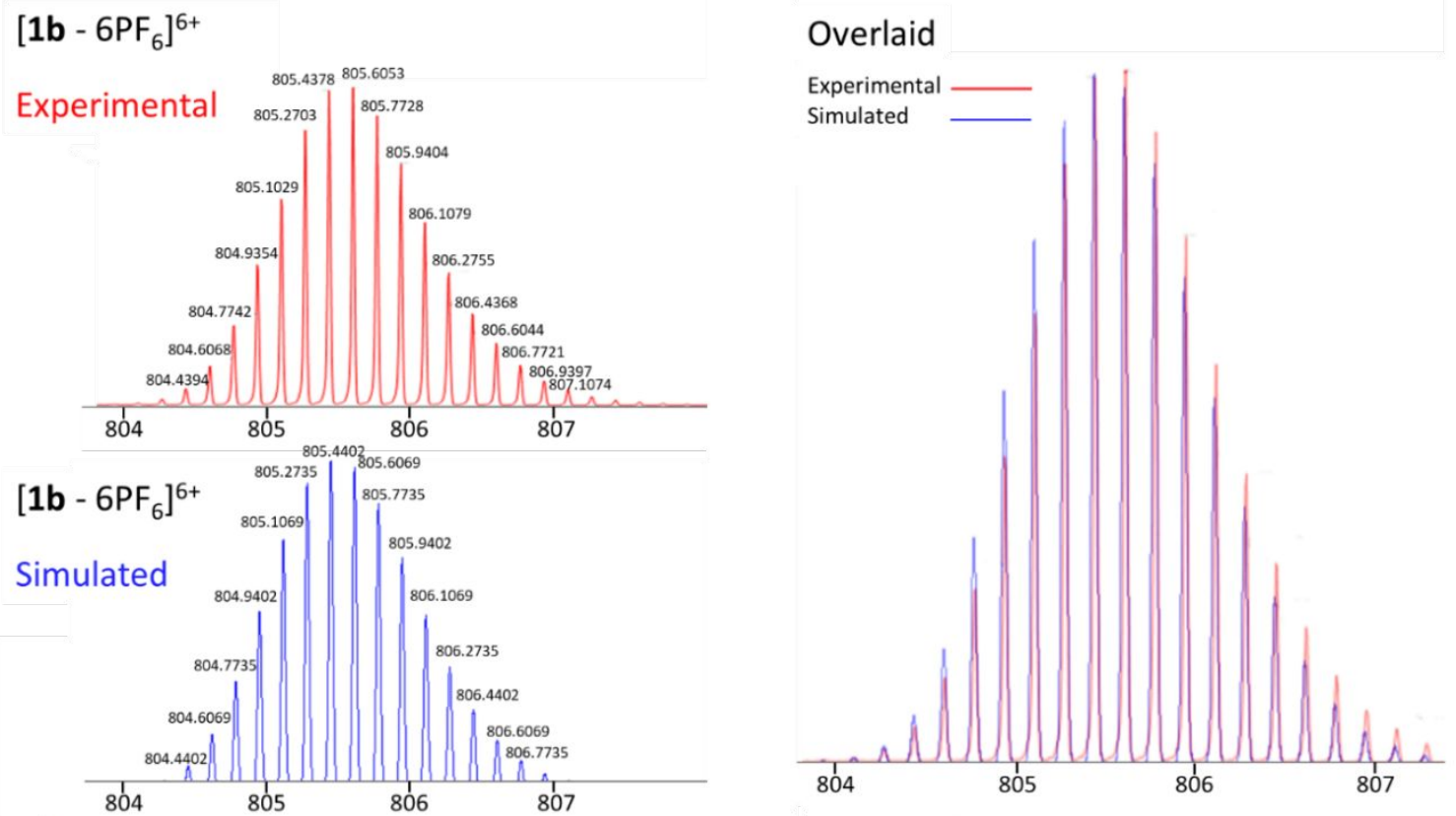

Figure S14: $\mathrm{ESI}(+)-\mathrm{MS}$ of $\mathbf{1 b}$ in $\mathrm{CH}_{3} \mathrm{CN}$. Experimental (red) and simulated (blue) spectra of [1b $\left.6 \mathrm{PF}_{6}\right]^{6+}$ showing isotopic distributions. An overlay of the experimental and simulated isotope pattern shows good agreement. 


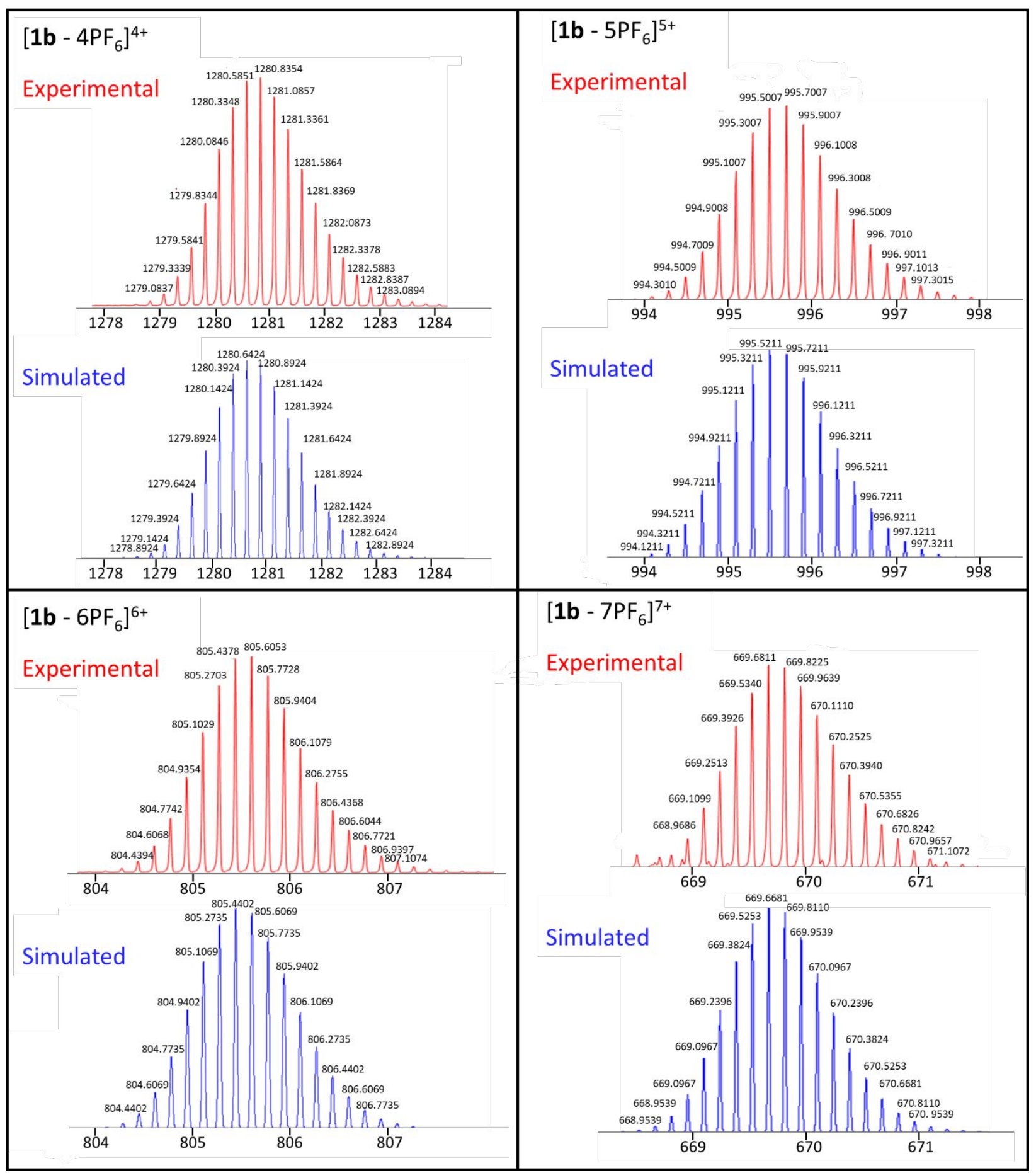

Figure S15: ESI(+)-MS peaks corresponding to the loss of 4 to $7 \mathrm{PF}_{6}{ }^{-}$anions from $1 \mathbf{b}$. Experimental (red) and simulated (blue) peaks are presented for $\left[\mathbf{1 b}-4 \mathrm{PF}_{6}\right]^{4+},\left[\mathbf{1 b}-5 \mathrm{PF}_{6}\right]^{5+},\left[\mathbf{1} \mathbf{b}-6 \mathrm{PF}_{6}\right]^{6+}$, and $\left[\mathbf{1 b}-7 \mathrm{PF}_{6}\right]^{7+}$. 


\section{X-Ray Crystallography}

X-ray Structure Determination. Crystals of 1a for XRD were grown by vapor diffusion of diethyl ether into an MeCN solution of 1a. After several days at $4{ }^{\circ} \mathrm{C}$, purple crystals and purple powder formed. The powder was removed as a suspension in the solvent mixture. The crystals were quickly placed in an oil-based cryoprotectant (Paratone oil) to prevent desolvation. Data for 1a were collected on a Bruker Smart APEX CCD diffractometer with graphite monochromatized Mo K $\alpha$ radiation $(\lambda=0.71073 \AA$ ) at $120 \mathrm{~K}$. As is common for porous structures, weak diffraction was observed at high angles even for relatively large crystals. The space group was found to be Pmmn (no. 59), and the intersecting mirrors determined the hexamer molecular site symmetry as $D_{2 h}$. The x-ray data for 1a were corrected for Lorenz effects and polarization, and absorption, the latter by a multi-scan (SADABS) ${ }^{12}$ method. The structure of 1a was solved by an intrinsic phasing method (SHELXT). ${ }^{13}$ All non-hydrogen atoms were refined $(\mathrm{SHELXL})^{14}$ based upon $\left(\mathrm{F}_{\mathrm{obs}}\right)^{\wedge} 2$. At an intermediate stage in the refinement, the difference-Fourier map helped locate all counter-ions as hexafluorophosphate, rather than triflate which was used in an early stage in the synthesis. Also located were several likely acetonitrile molecules and one diethyl ether molecule. An analysis of the very large void regions in the unit cell were examined with PLATON (CALC SOLV), where it was found that solvent accessible voids totaling over $4450 \AA^{3}$ were present. With an approximate acetonitrile volume of $83 \AA^{3}$, those voids were likely to contain over 50 acetonitrile molecules (or the equivalent combination of acetonitrile and diethyl ether molecules in the unit cell with its $Z^{\prime}=2$ hexamer molecules per unit cell). The PLATON ${ }^{15}$ result was consistent with the final model which employed one quarter the hexameric cation, five full-occupancy PF6- sites, eight acetonitrile sites and one ether site in the asymmetric unit. It is assumed that disordered

sites of solvents are present, but not included in the model. All hydrogen atom coordinates were calculated with idealized geometries (SHELXL). Scattering factors $\left(f_{0}, f^{\prime}, f^{\prime \prime}\right)$ are as described in SHELXL. Crystallographic data and final $R$ indices for $\mathbf{1 a}$ are given in Table S1. A summary of restraints used in the refined is provided here:

1. Very modest atomic displacement parameter restraints were employed:

ISOR .03

SIMU .05

DELU .05

2. Average geometries from the CSD were used to restrain geometries of all $\mathrm{PF}_{6}{ }^{-}$anions, all MeCN solvates, and the ethyl ether solvate:

DFIX 1.55 .01 P1 F1 P1 F2 P1 F3 P1 F4 P1 F5 P1 F6

SADI .01 F1 F2 F1 F4 F1 F5 F1 F6 F2 F3 F2 F5 F2 F6 
DFIX 1.13 .003 N21 C211 N22 C221 N23 C231 N24 C241 N26 C261 N25 C251 N27 C271 N28 C281

DFIX 1.45 .003 C212 C211 C222 C221 C232 C231 C242 C241 C262 C261 C252 C251 C272 C271 C282 C281

DFIX 2.58 .003 N21 C212 N22 C222 N23 C232 N24 C242 N26 C262 N25 C252 N27 C272 N28

$\mathrm{C} 282$

DFIX 1.42 .003031 C311 C311 C312

DFIX 2.37.003 031 C312 C311 C311_\$4

3. The small disordered region of cage, at $\mathrm{Pt}(2)$ and extending to its amino group, was restrained to be the same as that of $\mathrm{Pt}(1)$ [this follows atom $\mathrm{Pt}(1)$ ]:

SAME .005 PT2 N11 C37 C38 C39

4. Geometries of all $\mathrm{PF}_{6}{ }^{-}$anions were restrained to be the same [these follow atom $\mathrm{P}(1)$ ]:

SAME .01 P2 F7A F8A F8B F7B F9 F10

SAME .01 P3 F11A F12A F11B F12B F13A F13B

SAME .01 P4 F14 F15 F16 F17 F18A F18B

SAME .01 P5 F19 F20 F21 F22 F23 F24

5. $\mathrm{H}$ atoms all restrained to their sp2 or sp3 geometries, as in SHELXL (HFIX). The MeCN proton positions were fixed for the final cycles of refinement. 


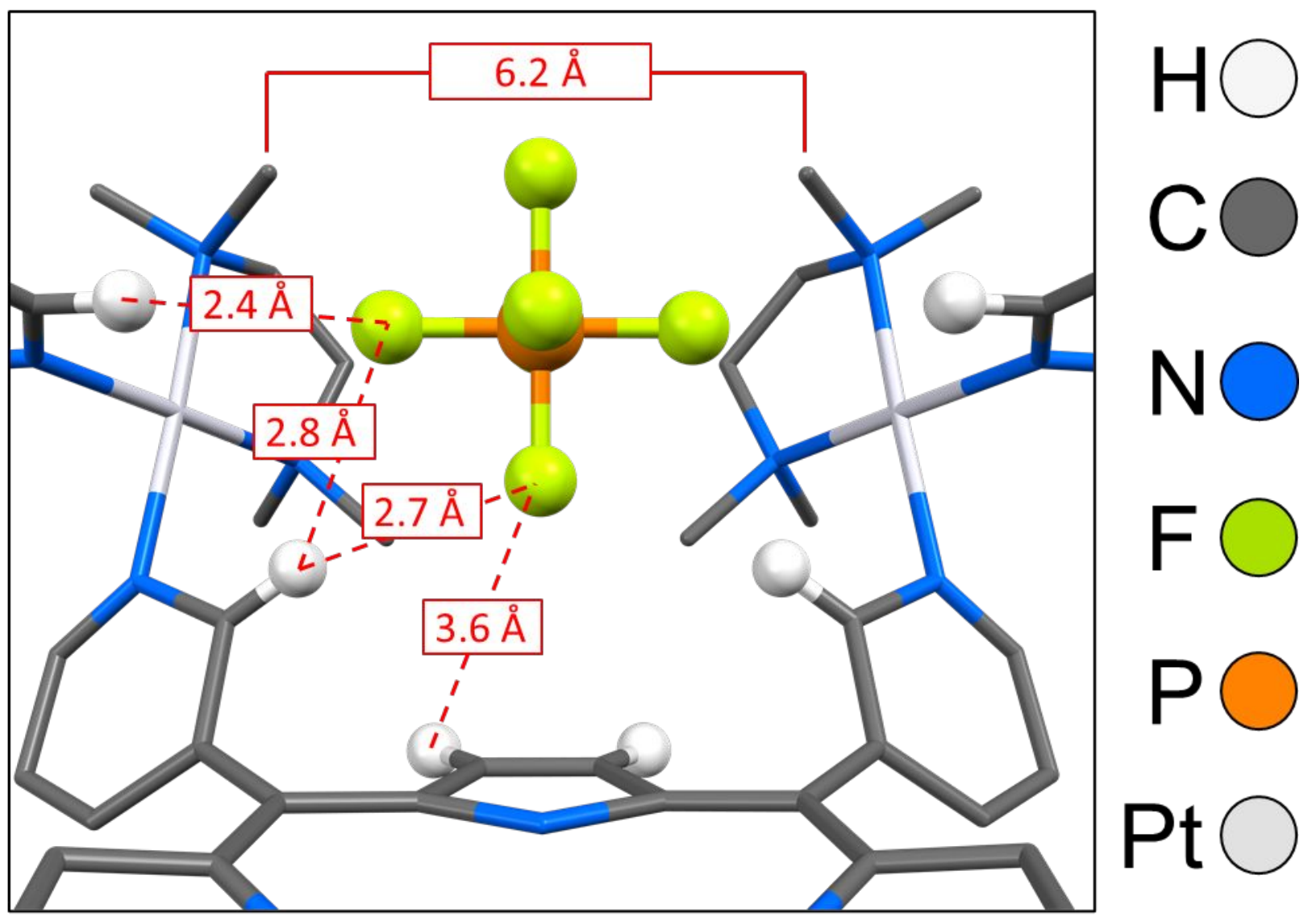

Figure S16: View from inside 1a in the solid state depicting close contacts between the 2-position hydrogen atoms of the 3-pyridyl groups and the fluorine atoms of an internally bound $\mathrm{PF}_{6}{ }^{-}$anion. All $\mathrm{CH}---\mathrm{F}$ distances are best regarded as approximations considering the relatively low quality of the diffraction data. The $\mathrm{C}---\mathrm{C}$ distance that defines the width of the apertures is also labelled, corresponding to a van der Waals separation of ca. 2.6 $\AA$. As with other distances in this structure, this $\mathrm{C}---\mathrm{C}$ distance should be regarded as approximate. 
Table S1. Crystal data and structure refinement for Cage 1a.

Identification code

Empirical formula

Formula weight

Temperature

Wavelength

Crystal system

Space group

Unit cell dimensions

Volume

Z

Density (calculated)

Absorption coefficient

$\mathrm{F}(000)$

Crystal size

Theta range for data collection

Index ranges

Reflections collected

Independent reflections

Completeness to theta $=17.079^{\circ}$

Absorption correction

Max. and min. transmission

Refinement method

Data / restraints / parameters

Goodness-of-fit on $\mathrm{F}^{2}$

Final R indices [I $>2 \operatorname{sigma}(\mathrm{I})]$

$\mathrm{R}$ indices (all data)

Extinction coefficient

Largest diff. peak and hole
Pt _TriCage_1a

C188 H229 F72 N50 O P12 Pt6

6115.38

120(2) K

$0.71073 \AA$

Orthorhombic

Pmmn

$\mathrm{a}=31.902(4) \AA$ $\alpha=90^{\circ}$.

$\mathrm{b}=21.840(3) \AA$ $\beta=90^{\circ}$.

$\mathrm{c}=19.751(3) \AA$ $\gamma=90^{\circ}$.

13761(3) $\AA^{3}$

2

$1.476 \mathrm{Mg} / \mathrm{m}^{3}$

$3.208 \mathrm{~mm}^{-1}$

6022

$0.320 \times 0.120 \times 0.070 \mathrm{~mm}^{3}$

1.530 to $17.079^{\circ}$.

$-26<=\mathrm{h}<=25,-17<=\mathrm{k}<=18,-16<=1<=16$

26217

$4332[\mathrm{R}(\mathrm{int})=0.1798]$

$99.4 \%$

Semi-empirical from equivalents

0.7446 and 0.5472

Full-matrix least-squares on $\mathrm{F}^{2}$

4332 / 411 / 419

1.161

$\mathrm{R} 1=0.1410, \mathrm{wR} 2=0.3606$

$\mathrm{R} 1=0.2590, \mathrm{wR} 2=0.4196$

$\mathrm{n} / \mathrm{a}$

1.873 and -1.092 e. $\AA^{-3}$ 
Table S2. Atomic coordinates $\left(x 10^{4}\right)$ and equivalent isotropic displacement parameters $\left(\AA^{2}\right.$ $x 10^{3}$ ) for $1 a . \quad U(e q)$ is defined as one third of the trace of the orthogonalized $U^{i j}$ tensor.

\begin{tabular}{|c|c|c|c|c|}
\hline & $\mathrm{x}$ & $\mathrm{y}$ & $\mathrm{z}$ & $\mathrm{U}(\mathrm{eq})$ \\
\hline $\operatorname{Pt}(1)$ & $5392(1)$ & $5600(1)$ & $1798(1)$ & $71(1)$ \\
\hline $\mathrm{N}(6)$ & 4983(11) & $5662(17)$ & $2598(17)$ & $75(12)$ \\
\hline$C(21)$ & 4930(13) & $5114(18)$ & $2950(20)$ & $76(17)$ \\
\hline$C(22)$ & $5163(13)$ & $6077(19)$ & $3178(19)$ & $74(16)$ \\
\hline$C(23)$ & $4616(12)$ & $5966(19)$ & $2410(20)$ & $58(13)$ \\
\hline$C(24)$ & 4701(14) & $6440(20)$ & $1840(20)$ & $81(15)$ \\
\hline$C(25)$ & $5150(14)$ & $6640(20)$ & $890(20)$ & $73(16)$ \\
\hline$C(26)$ & $4753(13)$ & $5814(19)$ & 830(20) & $59(15)$ \\
\hline $\mathrm{N}(7)$ & $4996(11)$ & $6167(16)$ & $1359(18)$ & $56(10)$ \\
\hline $\mathrm{N}(1)$ & 7500 & $5230(20)$ & $3550(30)$ & $72(17)$ \\
\hline $\mathrm{N}(2)$ & $6853(16)$ & $5670(20)$ & $4520(20)$ & $111(16)$ \\
\hline $\mathrm{N}(3)$ & 7500 & $6020(30)$ & $5480(30)$ & $100(20)$ \\
\hline$C(1)$ & $7292(11)$ & $4830(20)$ & $2550(20)$ & $71(16)$ \\
\hline$C(2)$ & $7155(17)$ & $5090(20)$ & $3160(30)$ & $84(16)$ \\
\hline$C(3)$ & $6717(16)$ & $5110(20)$ & $3400(30)$ & $81(16)$ \\
\hline $\mathrm{C}(4)$ & $6610(20)$ & $5520(30)$ & $4020(40)$ & $130(19)$ \\
\hline$C(5)$ & $6160(19)$ & $5370(20)$ & $4280(30)$ & $109(19)$ \\
\hline$C(6)$ & 6193(18) & $5650(20)$ & $4970(30)$ & $99(17)$ \\
\hline$C(7)$ & $6620(20)$ & $5880(30)$ & $5100(40)$ & $109(18)$ \\
\hline $\mathrm{C}(8)$ & $6751(19)$ & $6140(30)$ & $5610(30)$ & $101(18)$ \\
\hline$C(9)$ & $7170(20)$ & $6200(30)$ & $5840(30)$ & $102(18)$ \\
\hline$C(10)$ & $7307(13)$ & $6600(20)$ & $6440(30)$ & $100(20)$ \\
\hline $\mathrm{N}(4)$ & $5798(11)$ & $5087(19)$ & $2272(18)$ & $65(11)$ \\
\hline $\mathrm{C}(11)$ & $6116(14)$ & $5290(20)$ & $2590(20)$ & $54(13)$ \\
\hline$C(12)$ & $6369(16)$ & $4880(30)$ & $2920(30)$ & $82(16)$ \\
\hline$C(13)$ & $6368(16)$ & $4300(30)$ & $2890(20)$ & $82(16)$ \\
\hline$C(14)$ & $6037(14)$ & $3960(20)$ & $2520(20)$ & $76(15)$ \\
\hline$C(15)$ & $5761(13)$ & $4460(20)$ & $2190(20)$ & $50(13)$ \\
\hline $\mathrm{N}(5)$ & $5784(10)$ & $6849(14)$ & $6420(20)$ & $110(14)$ \\
\hline$C(16)$ & 6104(13) & $6742(16)$ & $5961(15)$ & $107(18)$ \\
\hline $\mathrm{C}(17)$ & $6419(10)$ & $6324(18)$ & $6110(20)$ & $92(17)$ \\
\hline
\end{tabular}




\begin{tabular}{|c|c|c|c|c|}
\hline$C(18)$ & $6414(11)$ & $6013(15)$ & $6730(20)$ & $150(20)$ \\
\hline$C(19)$ & $6094(15)$ & $6120(18)$ & $7189(17)$ & $160(20)$ \\
\hline$C(20)$ & $5779(11)$ & $6538(19)$ & $7038(18)$ & $150(20)$ \\
\hline $\operatorname{Pt}(2)$ & $5385(3)$ & 7500 & $6155(5)$ & $80(4)$ \\
\hline $\mathrm{N}(11)$ & $4945(12)$ & $8128(18)$ & $5850(20)$ & $190(20)$ \\
\hline$C(37)$ & $4744(17)$ & $8430(30)$ & $6380(30)$ & $250(40)$ \\
\hline$C(38)$ & $5151(18)$ & $8690(20)$ & $5480(40)$ & $280(50)$ \\
\hline$C(39)$ & $4685(17)$ & $7880(30)$ & $5350(30)$ & $300(40)$ \\
\hline $\mathrm{N}(8)$ & 7500 & $6584(18)$ & $403(18)$ & $18(12)$ \\
\hline $\mathrm{N}(9)$ & $6814(16)$ & 7500 & $420(20)$ & $58(16)$ \\
\hline$C(27)$ & $7276(11)$ & $5680(20)$ & $585(19)$ & $45(13)$ \\
\hline $\mathrm{C}(28)$ & $7189(13)$ & $6210(20)$ & $460(20)$ & $49(13)$ \\
\hline $\mathrm{C}(29)$ & $6724(14)$ & $6380(20)$ & $380(20)$ & $55(14)$ \\
\hline $\mathrm{C}(30)$ & $6610(14)$ & $6960(20)$ & $290(20)$ & $64(14)$ \\
\hline$C(31)$ & $6190(14)$ & $7182(16)$ & $160(20)$ & $66(16)$ \\
\hline $\mathrm{N}(10)$ & $5797(10)$ & $5507(17)$ & $989(17)$ & $52(11)$ \\
\hline$C(32)$ & $6089(13)$ & $6010(20)$ & $910(20)$ & $53(13)$ \\
\hline$C(33)$ & $6375(15)$ & $5950(20)$ & $430(20)$ & $66(14)$ \\
\hline$C(34)$ & $6373(16)$ & $5440(20)$ & $20(30)$ & $79(16)$ \\
\hline$C(35)$ & $6064(15)$ & $4970(20)$ & $20(20)$ & $80(16)$ \\
\hline$C(36)$ & $5782(13)$ & $5050(20)$ & $560(20)$ & $48(13)$ \\
\hline $\operatorname{Pt}(2 B)$ & $5356(4)$ & $7803(8)$ & 6032(8) & $69(5)$ \\
\hline $\mathrm{P}(1)$ & 5304(4) & $8762(6)$ & $8859(7)$ & $93(6)$ \\
\hline $\mathrm{F}(1)$ & $5353(8)$ & $9369(9)$ & $8462(13)$ & $220(20)$ \\
\hline $\mathrm{F}(2)$ & $5765(5)$ & $8583(11)$ & $8689(13)$ & 193(17) \\
\hline $\mathrm{F}(3)$ & $5247(8)$ & $8177(10)$ & $9282(12)$ & $137(12)$ \\
\hline $\mathrm{F}(4)$ & $4845(6)$ & $8919(14)$ & $9038(15)$ & 192(16) \\
\hline $\mathrm{F}(5)$ & $5152(8)$ & $8434(11)$ & $8202(11)$ & $165(14)$ \\
\hline $\mathrm{F}(6)$ & $5450(9)$ & $9075(12)$ & $9521(10)$ & $200(18)$ \\
\hline $\mathrm{P}(2)$ & $4172(10)$ & 7500 & $3443(15)$ & $238(19)$ \\
\hline $\mathrm{F}(7 \mathrm{~A})$ & $3969(17)$ & $8103(15)$ & $3190(30)$ & $280(50)$ \\
\hline $\mathrm{F}(8 \mathrm{~A})$ & $4518(16)$ & $7870(20)$ & $3820(30)$ & $390(80)$ \\
\hline $\mathrm{F}(8 \mathrm{~B})$ & $4358(16)$ & 6894(13) & $3700(20)$ & $140(30)$ \\
\hline $\mathrm{F}(7 \mathrm{~B})$ & $3841(14)$ & $7120(20)$ & $3070(30)$ & $250(50)$ \\
\hline $\mathrm{F}(9)$ & $4458(16)$ & 7500 & $2800(20)$ & $270(30)$ \\
\hline $\mathrm{F}(10)$ & $3897(17)$ & 7500 & $4090(20)$ & $320(40)$ \\
\hline
\end{tabular}




\begin{tabular}{|c|c|c|c|c|}
\hline $\mathrm{P}(3)$ & 5000 & 5000 & 5000 & $232(18)$ \\
\hline $\mathrm{F}(11 \mathrm{~A})$ & $4633(9)$ & $5411(17)$ & $5240(20)$ & $350(90)$ \\
\hline $\mathrm{F}(12 \mathrm{~A})$ & $5301(10)$ & $5322(16)$ & $5515(15)$ & $120(30)$ \\
\hline $\mathrm{F}(11 \mathrm{~B})$ & $5354(10)$ & $4576(16)$ & $4760(20)$ & $200(50)$ \\
\hline $\mathrm{F}(12 \mathrm{~B})$ & $4716(12)$ & $4674(18)$ & $4481(17)$ & $350(80)$ \\
\hline $\mathrm{F}(13 \mathrm{~A})$ & $5115(13)$ & $5499(14)$ & $4463(15)$ & $130(30)$ \\
\hline $\mathrm{F}(13 \mathrm{~B})$ & 4894(14) & $4505(14)$ & $5531(17)$ & $400(90)$ \\
\hline $\mathrm{P}(4)$ & $6014(8)$ & 7500 & $2500(12)$ & $165(13)$ \\
\hline $\mathrm{F}(14)$ & $5549(9)$ & 7500 & $2270(30)$ & $260(30)$ \\
\hline $\mathrm{F}(15)$ & $5875(16)$ & 7500 & $3261(14)$ & $310(40)$ \\
\hline $\mathrm{F}(16)$ & $6478(9)$ & 7500 & $2710(30)$ & $300(40)$ \\
\hline $\mathrm{F}(17)$ & $6166(16)$ & 7500 & $1760(16)$ & $270(30)$ \\
\hline $\mathrm{F}(18 \mathrm{~A})$ & $6001(10)$ & $8216(6)$ & $2501(17)$ & $220(40)$ \\
\hline $\mathrm{F}(18 \mathrm{~B})$ & $6032(11)$ & $6795(6)$ & $2507(16)$ & $210(40)$ \\
\hline $\mathrm{N}(27)$ & $6425(16)$ & 7500 & $4490(30)$ & $210(70)$ \\
\hline$C(271)$ & $6778(15)$ & 7500 & $4550(60)$ & $270(80)$ \\
\hline $\mathrm{C}(272)$ & $7226(15)$ & 7500 & $4670(100)$ & $280(90)$ \\
\hline $\mathrm{P}(5)$ & $3365(10)$ & $5468(15)$ & 1861(17) & $155(17)$ \\
\hline $\mathrm{F}(19)$ & $3653(16)$ & $5580(30)$ & $2480(20)$ & $190(30)$ \\
\hline $\mathrm{F}(20)$ & $3112(16)$ & $6060(20)$ & $2040(30)$ & $210(40)$ \\
\hline $\mathrm{F}(21)$ & $3089(18)$ & $5350(30)$ & $1240(20)$ & $270(50)$ \\
\hline $\mathrm{F}(22)$ & $3600(20)$ & $4875(19)$ & $1670(30)$ & $310(50)$ \\
\hline $\mathrm{F}(23)$ & $3062(17)$ & $5090(30)$ & $2330(30)$ & $270(40)$ \\
\hline $\mathrm{F}(24)$ & $3657(15)$ & $5840(20)$ & $1390(20)$ & $150(30)$ \\
\hline $\mathrm{N}(21)$ & $4180(30)$ & 7500 & $10260(30)$ & $430(70)$ \\
\hline $\mathrm{C}(211)$ & $3990(20)$ & 7500 & $10750(30)$ & $320(60)$ \\
\hline$C(212)$ & $3710(30)$ & 7500 & $11330(40)$ & $390(80)$ \\
\hline $\mathrm{N}(22)$ & $6710(40)$ & 7500 & $6990(30)$ & $520(90)$ \\
\hline$C(221)$ & $6620(40)$ & 7500 & $7540(30)$ & $420(70)$ \\
\hline$C(222)$ & $6530(40)$ & 7500 & $8260(30)$ & $330(70)$ \\
\hline $\mathrm{N}(23)$ & 7500 & $4690(40)$ & $6440(30)$ & $110(40)$ \\
\hline$C(231)$ & 7500 & $4400(40)$ & $5960(30)$ & $180(70)$ \\
\hline$C(232)$ & 7500 & $4070(50)$ & $5320(30)$ & $220(80)$ \\
\hline $\mathrm{N}(24)$ & $7800(20)$ & $6290(40)$ & $-1610(70)$ & $270(70)$ \\
\hline$C(241)$ & 7500 & $6020(40)$ & $-1570(40)$ & $280(60)$ \\
\hline$C(242)$ & $7110(20)$ & $5690(50)$ & $-1580(110)$ & $290(80)$ \\
\hline
\end{tabular}




\begin{tabular}{lllll}
$\mathrm{N}(25)$ & $3010(30)$ & 7500 & $7090(40)$ & $930(170)$ \\
$\mathrm{C}(251)$ & $3220(30)$ & 7500 & $6630(30)$ & $900(170)$ \\
$\mathrm{C}(252)$ & $3480(30)$ & 7500 & $6030(40)$ & $880(180)$ \\
$\mathrm{N}(26)$ & $4270(30)$ & 7500 & $7720(20)$ & $220(40)$ \\
$\mathrm{C}(261)$ & $4320(30)$ & 7500 & $8290(20)$ & $230(50)$ \\
$\mathrm{C}(262)$ & $4350(40)$ & 7500 & $9020(20)$ & $220(50)$ \\
$\mathrm{N}(28)$ & $6700(18)$ & $4210(20)$ & $1120(30)$ & $50(20)$ \\
$\mathrm{C}(281)$ & $6824(18)$ & $3770(20)$ & $890(30)$ & $30(20)$ \\
$\mathrm{C}(282)$ & $6950(40)$ & $3180(30)$ & $630(60)$ & $200(60)$ \\
$\mathrm{O}(31)$ & 7500 & 7500 & $2430(30)$ & $200(40)$ \\
$\mathrm{C}(311)$ & 7500 & $8043(1)$ & $2030(30)$ & $230(40)$ \\
$\mathrm{C}(312)$ & 7500 & $8585(1)$ & $2430(30)$ & $250(50)$ \\
& & & & \\
\hline
\end{tabular}


Table S3. Bond lengths $[\AA ̊]$ and angles $\left[^{\circ}\right]$ for 1 a.

\begin{tabular}{|c|c|c|c|}
\hline $\operatorname{Pt}(1)-N(4)$ & $1.95(4)$ & $\mathrm{C}(1)-\mathrm{C}(1) \# 1$ & $1.32(7)$ \\
\hline $\operatorname{Pt}(1)-\mathrm{N}(7)$ & $1.97(3)$ & $\mathrm{C}(1)-\mathrm{C}(2)$ & $1.40(6)$ \\
\hline $\operatorname{Pt}(1)-\mathrm{N}(10)$ & $2.06(3)$ & $\mathrm{C}(1)-\mathrm{H}(1)$ & 0.9500 \\
\hline $\operatorname{Pt}(1)-\mathrm{N}(6)$ & $2.06(3)$ & $\mathrm{C}(2)-\mathrm{C}(3)$ & $1.48(6)$ \\
\hline $\mathrm{N}(6)-\mathrm{C}(21)$ & $1.39(4)$ & $\mathrm{C}(3)-\mathrm{C}(12)$ & $1.54(6)$ \\
\hline $\mathrm{N}(6)-\mathrm{C}(23)$ & $1.40(4)$ & $C(3)-C(4)$ & $1.56(7)$ \\
\hline $\mathrm{N}(6)-\mathrm{C}(22)$ & $1.57(4)$ & $C(4)-C(5)$ & $1.56(7)$ \\
\hline $\mathrm{C}(21)-\mathrm{H}(21 \mathrm{~A})$ & 0.9800 & $C(5)-C(6)$ & $1.50(7)$ \\
\hline $\mathrm{C}(21)-\mathrm{H}(21 \mathrm{~B})$ & 0.9800 & $\mathrm{C}(5)-\mathrm{H}(5)$ & 0.9500 \\
\hline $\mathrm{C}(21)-\mathrm{H}(21 \mathrm{C})$ & 0.9800 & $C(6)-C(7)$ & $1.46(7)$ \\
\hline $\mathrm{C}(22)-\mathrm{H}(22 \mathrm{~A})$ & 0.9800 & $\mathrm{C}(6)-\mathrm{H}(6)$ & 0.9500 \\
\hline $\mathrm{C}(22)-\mathrm{H}(22 \mathrm{~B})$ & 0.9800 & $C(7)-C(8)$ & $1.24(7)$ \\
\hline $\mathrm{C}(22)-\mathrm{H}(22 \mathrm{C})$ & 0.9800 & $\mathrm{C}(8)-\mathrm{C}(9)$ & $1.41(7)$ \\
\hline$C(23)-C(24)$ & $1.55(5)$ & $\mathrm{C}(8)-\mathrm{C}(17)$ & $1.51(6)$ \\
\hline $\mathrm{C}(23)-\mathrm{H}(23 \mathrm{~A})$ & 0.9900 & $C(9)-C(10)$ & $1.53(6)$ \\
\hline $\mathrm{C}(23)-\mathrm{H}(23 \mathrm{~B})$ & 0.9900 & $C(10)-C(10) \# 1$ & $1.23(8)$ \\
\hline $\mathrm{C}(24)-\mathrm{N}(7)$ & $1.46(5)$ & $\mathrm{C}(10)-\mathrm{H}(10)$ & 0.9500 \\
\hline $\mathrm{C}(24)-\mathrm{H}(24 \mathrm{~A})$ & 0.9900 & $\mathrm{~N}(4)-\mathrm{C}(11)$ & $1.27(5)$ \\
\hline $\mathrm{C}(24)-\mathrm{H}(24 \mathrm{~B})$ & 0.9900 & $\mathrm{~N}(4)-\mathrm{C}(15)$ & $1.39(4)$ \\
\hline$C(25)-N(7)$ & $1.48(5)$ & $\mathrm{C}(11)-\mathrm{C}(12)$ & $1.36(6)$ \\
\hline $\mathrm{C}(25)-\mathrm{H}(25 \mathrm{~A})$ & 0.9800 & $\mathrm{C}(11)-\mathrm{H}(11)$ & 0.9500 \\
\hline $\mathrm{C}(25)-\mathrm{H}(25 \mathrm{~B})$ & 0.9800 & $\mathrm{C}(12)-\mathrm{C}(13)$ & $1.26(6)$ \\
\hline $\mathrm{C}(25)-\mathrm{H}(25 \mathrm{C})$ & 0.9800 & $\mathrm{C}(13)-\mathrm{C}(14)$ & $1.50(6)$ \\
\hline$C(26)-N(7)$ & $1.52(4)$ & $\mathrm{C}(13)-\mathrm{H}(13)$ & 0.9500 \\
\hline $\mathrm{C}(26)-\mathrm{H}(26 \mathrm{~A})$ & 0.9800 & $C(14)-C(15)$ & $1.55(5)$ \\
\hline $\mathrm{C}(26)-\mathrm{H}(26 \mathrm{~B})$ & 0.9800 & $\mathrm{C}(14)-\mathrm{H}(14)$ & 0.9500 \\
\hline $\mathrm{C}(26)-\mathrm{H}(26 \mathrm{C})$ & 0.9800 & $\mathrm{C}(15)-\mathrm{H}(15)$ & 0.9500 \\
\hline $\mathrm{N}(1)-\mathrm{C}(2)$ & $1.38(5)$ & $\mathrm{N}(5)-\mathrm{C}(16)$ & 1.3900 \\
\hline $\mathrm{N}(1)-\mathrm{C}(2) \# 1$ & $1.38(5)$ & $\mathrm{N}(5)-\mathrm{C}(20)$ & 1.3900 \\
\hline $\mathrm{N}(2)-\mathrm{C}(4)$ & $1.31(7)$ & $\mathrm{N}(5)-\operatorname{Pt}(2)$ & $1.98(3)$ \\
\hline $\mathrm{N}(2)-\mathrm{C}(7)$ & $1.44(6)$ & $C(16)-C(17)$ & 1.3900 \\
\hline $\mathrm{N}(2)-\mathrm{H}(2 \mathrm{~N})$ & 0.8800 & $\mathrm{C}(16)-\mathrm{H}(16)$ & 0.9500 \\
\hline $\mathrm{N}(3)-\mathrm{C}(9)$ & $1.33(6)$ & $\mathrm{C}(17)-\mathrm{C}(18)$ & 1.3900 \\
\hline $\mathrm{N}(3)-\mathrm{C}(9) \# 1$ & $1.33(6)$ & $\mathrm{C}(18)-\mathrm{C}(19)$ & 1.3900 \\
\hline
\end{tabular}




\begin{tabular}{|c|c|c|c|}
\hline $\mathrm{C}(18)-\mathrm{H}(18)$ & 0.9500 & $C(33)-C(34)$ & $1.39(5)$ \\
\hline$C(19)-C(20)$ & 1.3900 & $C(34)-C(35)$ & $1.42(6)$ \\
\hline $\mathrm{C}(19)-\mathrm{H}(19)$ & 0.9500 & $\mathrm{C}(34)-\mathrm{H}(34)$ & 0.9500 \\
\hline $\mathrm{C}(20)-\mathrm{H}(20)$ & 0.9500 & $C(35)-C(36)$ & $1.40(5)$ \\
\hline $\operatorname{Pt}(2)-\mathrm{N}(11) \# 2$ & $2.05(3)$ & $\mathrm{C}(35)-\mathrm{H}(35)$ & 0.9500 \\
\hline $\operatorname{Pt}(2)-\mathrm{N}(11)$ & $2.05(3)$ & $\mathrm{C}(36)-\mathrm{H}(36)$ & 0.9500 \\
\hline $\mathrm{N}(11)-\mathrm{C}(37)$ & $1.40(4)$ & $\mathrm{P}(1)-\mathrm{F}(3)$ & $1.539(10)$ \\
\hline N(11)-C(39) & $1.40(4)$ & $\mathrm{P}(1)-\mathrm{F}(4)$ & $1.544(10)$ \\
\hline $\mathrm{N}(11)-\mathrm{C}(38)$ & $1.57(4)$ & $\mathrm{P}(1)-\mathrm{F}(6)$ & $1.546(10)$ \\
\hline $\mathrm{C}(37)-\mathrm{H}(37 \mathrm{~A})$ & 0.9800 & $\mathrm{P}(1)-\mathrm{F}(1)$ & $1.548(10)$ \\
\hline C(37)-H(37B) & 0.9800 & $\mathrm{P}(1)-\mathrm{F}(2)$ & $1.559(10)$ \\
\hline $\mathrm{C}(37)-\mathrm{H}(37 \mathrm{C})$ & 0.9800 & $\mathrm{P}(1)-\mathrm{F}(5)$ & $1.560(9)$ \\
\hline $\mathrm{C}(38)-\mathrm{H}(38 \mathrm{~A})$ & 0.9800 & $\mathrm{P}(2)-\mathrm{F}(8 \mathrm{~B})$ & $1.536(13)$ \\
\hline $\mathrm{C}(38)-\mathrm{H}(38 \mathrm{~B})$ & 0.9800 & $\mathrm{P}(2)-\mathrm{F}(7 \mathrm{~B})$ & $1.541(13)$ \\
\hline $\mathrm{C}(38)-\mathrm{H}(38 \mathrm{C})$ & 0.9800 & $\mathrm{P}(2)-\mathrm{F}(10)$ & $1.543(13)$ \\
\hline $\mathrm{C}(39)-\mathrm{C}(39) \# 2$ & $1.66(11)$ & $\mathrm{P}(2)-\mathrm{F}(7 \mathrm{~A})$ & $1.550(13)$ \\
\hline C(39)-H(39A) & 0.9900 & $\mathrm{P}(2)-\mathrm{F}(9)$ & $1.564(13)$ \\
\hline C(39)-H(39B) & 0.9900 & $\mathrm{P}(2)-\mathrm{F}(8 \mathrm{~A})$ & $1.566(13)$ \\
\hline $\mathrm{N}(8)-\mathrm{C}(28)$ & $1.28(4)$ & $\mathrm{P}(3)-\mathrm{F}(11 \mathrm{~B})$ & $1.537(13)$ \\
\hline $\mathrm{N}(8)-\mathrm{C}(28) \# 1$ & $1.28(4)$ & $\mathrm{P}(3)-\mathrm{F}(12 \mathrm{~B})$ & $1.542(13)$ \\
\hline $\mathrm{N}(9)-\mathrm{C}(30) \# 2$ & $1.38(5)$ & $\mathrm{P}(3)-\mathrm{F}(13 \mathrm{~B})$ & $1.543(13)$ \\
\hline $\mathrm{N}(9)-\mathrm{C}(30)$ & $1.38(5)$ & $\mathrm{P}(3)-\mathrm{F}(11 \mathrm{~A})$ & $1.549(13)$ \\
\hline $\mathrm{N}(9)-\mathrm{H}(9 \mathrm{~N})$ & 0.8800 & $\mathrm{P}(3)-\mathrm{F}(13 \mathrm{~A})$ & $1.564(13)$ \\
\hline$C(27)-C(28)$ & $1.22(4)$ & $\mathrm{P}(3)-\mathrm{F}(12 \mathrm{~A})$ & $1.566(13)$ \\
\hline$C(27)-C(27) \# 1$ & $1.43(7)$ & $P(4)-F(16)$ & $1.537(13)$ \\
\hline $\mathrm{C}(27)-\mathrm{H}(27)$ & 0.9500 & $\mathrm{P}(4)-\mathrm{F}(17)$ & $1.540(13)$ \\
\hline$C(28)-C(29)$ & $1.53(5)$ & $\mathrm{P}(4)-\mathrm{F}(18 \mathrm{~B})$ & $1.541(12)$ \\
\hline$C(29)-C(30)$ & $1.33(5)$ & $\mathrm{P}(4)-\mathrm{F}(18 \mathrm{~B}) \# 2$ & $1.541(12)$ \\
\hline$C(29)-C(33)$ & $1.45(5)$ & $\mathrm{P}(4)-\mathrm{F}(14)$ & $1.549(13)$ \\
\hline$C(30)-C(31)$ & $1.45(5)$ & $\mathrm{P}(4)-\mathrm{F}(18 \mathrm{~A}) \# 2$ & $1.564(12)$ \\
\hline$C(31)-C(31) \# 2$ & $1.39(7)$ & $\mathrm{P}(4)-\mathrm{F}(18 \mathrm{~A})$ & $1.564(12)$ \\
\hline $\mathrm{C}(31)-\mathrm{H}(31)$ & 0.9500 & $P(4)-F(15)$ & $1.566(13)$ \\
\hline $\mathrm{N}(10)-\mathrm{C}(36)$ & $1.31(4)$ & $\mathrm{F}(18 \mathrm{~A})-\mathrm{F}(18 \mathrm{~B}) \# 2$ & $0.10(4)$ \\
\hline $\mathrm{N}(10)-\mathrm{C}(32)$ & $1.44(5)$ & $\mathrm{N}(27)-\mathrm{C}(271)$ & $1.130(3)$ \\
\hline$C(32)-C(33)$ & $1.33(5)$ & $\mathrm{C}(271)-\mathrm{C}(272)$ & $1.450(3)$ \\
\hline $\mathrm{C}(32)-\mathrm{H}(32 \mathrm{~A})$ & 0.9500 & $\mathrm{C}(272)-\mathrm{C}(272) \# 3$ & $1.75(9)$ \\
\hline
\end{tabular}




\begin{tabular}{|c|c|c|c|}
\hline $\mathrm{C}(272)-\mathrm{H}(271)$ & 1.1662 & $\mathrm{C}(241)-\mathrm{C}(242)$ & $1.451(3)$ \\
\hline $\mathrm{C}(272)-\mathrm{H}(272)$ & 1.1175 & $\mathrm{C}(241)-\mathrm{H}(243)$ & 1.5643 \\
\hline $\mathrm{C}(272)-\mathrm{H}(273)$ & 0.6952 & $\mathrm{C}(242)-\mathrm{H}(241)$ & 0.8530 \\
\hline $\mathrm{C}(272)-\mathrm{H}(273) \# 2$ & $0.70(19)$ & $\mathrm{C}(242)-\mathrm{H}(242)$ & 1.0671 \\
\hline $\mathrm{P}(5)-\mathrm{F}(21)$ & $1.537(13)$ & $\mathrm{C}(242)-\mathrm{H}(243)$ & 0.8700 \\
\hline $\mathrm{P}(5)-\mathrm{F}(22)$ & $1.541(13)$ & $\mathrm{N}(25)-\mathrm{C}(251)$ & $1.130(3)$ \\
\hline$P(5)-F(24)$ & $1.541(13)$ & $C(251)-C(252)$ & $1.450(3)$ \\
\hline $\mathrm{P}(5)-\mathrm{F}(19)$ & $1.547(13)$ & $\mathrm{C}(252)-\mathrm{H}(251)$ & 1.2280 \\
\hline $\mathrm{P}(5)-\mathrm{F}(20)$ & $1.564(13)$ & $\mathrm{C}(252)-\mathrm{H}(252)$ & 1.0885 \\
\hline $\mathrm{P}(5)-\mathrm{F}(23)$ & $1.565(13)$ & $\mathrm{C}(252)-\mathrm{H}(253)$ & 1.0840 \\
\hline $\mathrm{N}(21)-\mathrm{C}(211)$ & $1.131(3)$ & $\mathrm{N}(26)-\mathrm{C}(261)$ & $1.130(3)$ \\
\hline $\mathrm{C}(211)-\mathrm{C}(212)$ & $1.451(3)$ & $C(261)-C(262)$ & $1.450(3)$ \\
\hline $\mathrm{C}(212)-\mathrm{H}(211)$ & 1.1259 & $\mathrm{C}(262)-\mathrm{H}(261)$ & 0.8495 \\
\hline $\mathrm{C}(212)-\mathrm{H}(212)$ & 0.9398 & $\mathrm{C}(262)-\mathrm{H}(262)$ & 0.9619 \\
\hline $\mathrm{C}(212)-\mathrm{H}(213)$ & 1.0499 & $\mathrm{C}(262)-\mathrm{H}(263)$ & 1.0564 \\
\hline $\mathrm{C}(212)-\mathrm{H}(212) \# 2$ & $0.94(7)$ & $\mathrm{C}(262)-\mathrm{H}(261) \# 2$ & $0.85(9)$ \\
\hline $\mathrm{N}(22)-\mathrm{C}(221)$ & $1.130(3)$ & $\mathrm{C}(262)-\mathrm{H}(262) \# 2$ & $0.96(2)$ \\
\hline $\mathrm{C}(221)-\mathrm{C}(222)$ & $1.450(3)$ & $\mathrm{N}(28)-\mathrm{C}(281)$ & $1.131(3)$ \\
\hline $\mathrm{C}(222)-\mathrm{H}(221)$ & 1.0046 & $\mathrm{C}(281)-\mathrm{C}(282)$ & $1.451(3)$ \\
\hline $\mathrm{C}(222)-\mathrm{H}(222)$ & 1.0481 & $\mathrm{C}(282)-\mathrm{H}(281)$ & 0.9471 \\
\hline $\mathrm{C}(222)-\mathrm{H}(223)$ & 1.0812 & $\mathrm{C}(282)-\mathrm{H}(282)$ & 0.9814 \\
\hline $\mathrm{C}(222)-\mathrm{H}(221) \# 2$ & $1.00(11)$ & $\mathrm{C}(282)-\mathrm{H}(283)$ & 0.9503 \\
\hline $\mathrm{N}(23)-\mathrm{C}(231)$ & $1.131(3)$ & $\mathrm{O}(31)-\mathrm{C}(311) \# 3$ & $1.420(3)$ \\
\hline$C(231)-C(232)$ & $1.451(3)$ & $\mathrm{O}(31)-\mathrm{C}(311)$ & $1.420(3)$ \\
\hline $\mathrm{C}(232)-\mathrm{H}(231)$ & 1.0334 & $\mathrm{C}(311)-\mathrm{C}(312)$ & $1.420(3)$ \\
\hline $\mathrm{C}(232)-\mathrm{H}(232)$ & 0.9969 & $\mathrm{C}(311)-\mathrm{H}(311)$ & 0.9900 \\
\hline $\mathrm{C}(232)-\mathrm{H}(233)$ & 0.9056 & $\mathrm{C}(311)-\mathrm{H}(312)$ & 0.9900 \\
\hline $\mathrm{C}(232)-\mathrm{H}(231) \# 1$ & $1.03(8)$ & $\mathrm{C}(312)-\mathrm{H}(313)$ & 0.9800 \\
\hline $\mathrm{C}(232)-\mathrm{H}(232) \# 1$ & $1.00(4)$ & $\mathrm{C}(312)-\mathrm{H}(314)$ & 0.9800 \\
\hline $\mathrm{C}(232)-\mathrm{H}(233) \# 1$ & $0.91(11)$ & $\mathrm{C}(312)-\mathrm{H}(315)$ & 0.9800 \\
\hline $\mathrm{N}(24)-\mathrm{C}(241)$ & $1.131(3)$ & & \\
\hline $\mathrm{N}(4)-\operatorname{Pt}(1)-\mathrm{N}(7)$ & $175.9(16)$ & $\mathrm{N}(7)-\operatorname{Pt}(1)-\mathrm{N}(6)$ & $83.6(15)$ \\
\hline $\mathrm{N}(4)-\mathrm{Pt}(1)-\mathrm{N}(10)$ & $84.3(14)$ & $\mathrm{N}(10)-\mathrm{Pt}(1)-\mathrm{N}(6)$ & $178.0(15)$ \\
\hline $\mathrm{N}(7)-\mathrm{Pt}(1)-\mathrm{N}(10)$ & $97.1(14)$ & $\mathrm{C}(21)-\mathrm{N}(6)-\mathrm{C}(23)$ & $116(3)$ \\
\hline $\mathrm{N}(4)-\operatorname{Pt}(1)-\mathrm{N}(6)$ & $95.1(14)$ & $\mathrm{C}(21)-\mathrm{N}(6)-\mathrm{C}(22)$ & $100(3)$ \\
\hline
\end{tabular}




\begin{tabular}{|c|c|c|c|}
\hline $\mathrm{C}(23)-\mathrm{N}(6)-\mathrm{C}(22)$ & $103(3)$ & $\mathrm{H}(26 \mathrm{~A})-\mathrm{C}(26)-\mathrm{H}(26 \mathrm{~B})$ & 109.5 \\
\hline $\mathrm{C}(21)-\mathrm{N}(6)-\mathrm{Pt}(1)$ & $114(3)$ & $\mathrm{N}(7)-\mathrm{C}(26)-\mathrm{H}(26 \mathrm{C})$ & 109.5 \\
\hline $\mathrm{C}(23)-\mathrm{N}(6)-\operatorname{Pt}(1)$ & $111(3)$ & $\mathrm{H}(26 \mathrm{~A})-\mathrm{C}(26)-\mathrm{H}(26 \mathrm{C})$ & 109.5 \\
\hline $\mathrm{C}(22)-\mathrm{N}(6)-\mathrm{Pt}(1)$ & $111(2)$ & $\mathrm{H}(26 \mathrm{~B})-\mathrm{C}(26)-\mathrm{H}(26 \mathrm{C})$ & 109.5 \\
\hline $\mathrm{N}(6)-\mathrm{C}(21)-\mathrm{H}(21 \mathrm{~A})$ & 109.5 & $\mathrm{C}(24)-\mathrm{N}(7)-\mathrm{C}(25)$ & $110(3)$ \\
\hline $\mathrm{N}(6)-\mathrm{C}(21)-\mathrm{H}(21 \mathrm{~B})$ & 109.5 & $C(24)-N(7)-C(26)$ & $109(3)$ \\
\hline $\mathrm{H}(21 \mathrm{~A})-\mathrm{C}(21)-\mathrm{H}(21 \mathrm{~B})$ & 109.5 & $\mathrm{C}(25)-\mathrm{N}(7)-\mathrm{C}(26)$ & $95(3)$ \\
\hline $\mathrm{N}(6)-\mathrm{C}(21)-\mathrm{H}(21 \mathrm{C})$ & 109.5 & $\mathrm{C}(24)-\mathrm{N}(7)-\mathrm{Pt}(1)$ & $112(3)$ \\
\hline $\mathrm{H}(21 \mathrm{~A})-\mathrm{C}(21)-\mathrm{H}(21 \mathrm{C})$ & 109.5 & $\mathrm{C}(25)-\mathrm{N}(7)-\operatorname{Pt}(1)$ & $120(3)$ \\
\hline $\mathrm{H}(21 \mathrm{~B})-\mathrm{C}(21)-\mathrm{H}(21 \mathrm{C})$ & 109.5 & $\mathrm{C}(26)-\mathrm{N}(7)-\mathrm{Pt}(1)$ & $108(3)$ \\
\hline $\mathrm{N}(6)-\mathrm{C}(22)-\mathrm{H}(22 \mathrm{~A})$ & 109.5 & $\mathrm{C}(2)-\mathrm{N}(1)-\mathrm{C}(2) \# 1$ & $105(6)$ \\
\hline $\mathrm{N}(6)-\mathrm{C}(22)-\mathrm{H}(22 \mathrm{~B})$ & 109.5 & $\mathrm{C}(4)-\mathrm{N}(2)-\mathrm{C}(7)$ & $112(6)$ \\
\hline $\mathrm{H}(22 \mathrm{~A})-\mathrm{C}(22)-\mathrm{H}(22 \mathrm{~B})$ & 109.5 & $\mathrm{C}(4)-\mathrm{N}(2)-\mathrm{H}(2 \mathrm{~N})$ & 124.2 \\
\hline $\mathrm{N}(6)-\mathrm{C}(22)-\mathrm{H}(22 \mathrm{C})$ & 109.5 & $\mathrm{C}(7)-\mathrm{N}(2)-\mathrm{H}(2 \mathrm{~N})$ & 124.2 \\
\hline $\mathrm{H}(22 \mathrm{~A})-\mathrm{C}(22)-\mathrm{H}(22 \mathrm{C})$ & 109.5 & $\mathrm{C}(9)-\mathrm{N}(3)-\mathrm{C}(9) \# 1$ & $105(8)$ \\
\hline $\mathrm{H}(22 \mathrm{~B})-\mathrm{C}(22)-\mathrm{H}(22 \mathrm{C})$ & 109.5 & $\mathrm{C}(1) \# 1-\mathrm{C}(1)-\mathrm{C}(2)$ & $108(3)$ \\
\hline $\mathrm{N}(6)-\mathrm{C}(23)-\mathrm{C}(24)$ & $111(3)$ & $\mathrm{C}(1) \# 1-\mathrm{C}(1)-\mathrm{H}(1)$ & 125.9 \\
\hline $\mathrm{N}(6)-\mathrm{C}(23)-\mathrm{H}(23 \mathrm{~A})$ & 109.4 & $\mathrm{C}(2)-\mathrm{C}(1)-\mathrm{H}(1)$ & 125.9 \\
\hline $\mathrm{C}(24)-\mathrm{C}(23)-\mathrm{H}(23 \mathrm{~A})$ & 109.4 & $\mathrm{~N}(1)-\mathrm{C}(2)-\mathrm{C}(1)$ & $109(5)$ \\
\hline $\mathrm{N}(6)-\mathrm{C}(23)-\mathrm{H}(23 \mathrm{~B})$ & 109.4 & $\mathrm{~N}(1)-\mathrm{C}(2)-\mathrm{C}(3)$ & $125(5)$ \\
\hline $\mathrm{C}(24)-\mathrm{C}(23)-\mathrm{H}(23 \mathrm{~B})$ & 109.4 & $C(1)-C(2)-C(3)$ & $126(5)$ \\
\hline $\mathrm{H}(23 \mathrm{~A})-\mathrm{C}(23)-\mathrm{H}(23 \mathrm{~B})$ & 108.0 & $\mathrm{C}(2)-\mathrm{C}(3)-\mathrm{C}(12)$ & $118(5)$ \\
\hline $\mathrm{N}(7)-\mathrm{C}(24)-\mathrm{C}(23)$ & $109(4)$ & $C(2)-C(3)-C(4)$ & $119(5)$ \\
\hline $\mathrm{N}(7)-\mathrm{C}(24)-\mathrm{H}(24 \mathrm{~A})$ & 110.0 & $C(12)-C(3)-C(4)$ & $120(5)$ \\
\hline $\mathrm{C}(23)-\mathrm{C}(24)-\mathrm{H}(24 \mathrm{~A})$ & 110.0 & $\mathrm{~N}(2)-\mathrm{C}(4)-\mathrm{C}(5)$ & $110(6)$ \\
\hline $\mathrm{N}(7)-\mathrm{C}(24)-\mathrm{H}(24 \mathrm{~B})$ & 110.0 & $\mathrm{~N}(2)-\mathrm{C}(4)-\mathrm{C}(3)$ & $127(6)$ \\
\hline $\mathrm{C}(23)-\mathrm{C}(24)-\mathrm{H}(24 \mathrm{~B})$ & 110.0 & $C(5)-C(4)-C(3)$ & $110(6)$ \\
\hline $\mathrm{H}(24 \mathrm{~A})-\mathrm{C}(24)-\mathrm{H}(24 \mathrm{~B})$ & 108.4 & $C(6)-C(5)-C(4)$ & $98(5)$ \\
\hline $\mathrm{N}(7)-\mathrm{C}(25)-\mathrm{H}(25 \mathrm{~A})$ & 109.5 & $\mathrm{C}(6)-\mathrm{C}(5)-\mathrm{H}(5)$ & 130.8 \\
\hline $\mathrm{N}(7)-\mathrm{C}(25)-\mathrm{H}(25 \mathrm{~B})$ & 109.5 & $\mathrm{C}(4)-\mathrm{C}(5)-\mathrm{H}(5)$ & 130.8 \\
\hline $\mathrm{H}(25 \mathrm{~A})-\mathrm{C}(25)-\mathrm{H}(25 \mathrm{~B})$ & 109.5 & $C(7)-C(6)-C(5)$ & $111(6)$ \\
\hline $\mathrm{N}(7)-\mathrm{C}(25)-\mathrm{H}(25 \mathrm{C})$ & 109.5 & $\mathrm{C}(7)-\mathrm{C}(6)-\mathrm{H}(6)$ & 124.3 \\
\hline $\mathrm{H}(25 \mathrm{~A})-\mathrm{C}(25)-\mathrm{H}(25 \mathrm{C})$ & 109.5 & $\mathrm{C}(5)-\mathrm{C}(6)-\mathrm{H}(6)$ & 124.3 \\
\hline $\mathrm{H}(25 \mathrm{~B})-\mathrm{C}(25)-\mathrm{H}(25 \mathrm{C})$ & 109.5 & $\mathrm{C}(8)-\mathrm{C}(7)-\mathrm{N}(2)$ & $128(7)$ \\
\hline $\mathrm{N}(7)-\mathrm{C}(26)-\mathrm{H}(26 \mathrm{~A})$ & 109.5 & $\mathrm{C}(8)-\mathrm{C}(7)-\mathrm{C}(6)$ & $128(7)$ \\
\hline $\mathrm{N}(7)-\mathrm{C}(26)-\mathrm{H}(26 \mathrm{~B})$ & 109.5 & $\mathrm{~N}(2)-\mathrm{C}(7)-\mathrm{C}(6)$ & $104(6)$ \\
\hline
\end{tabular}




\begin{tabular}{|c|c|c|c|}
\hline $\mathrm{C}(7)-\mathrm{C}(8)-\mathrm{C}(9)$ & $129(7)$ & $\mathrm{C}(17)-\mathrm{C}(18)-\mathrm{C}(19)$ & 120.0 \\
\hline$C(7)-C(8)-C(17)$ & $115(6)$ & $\mathrm{C}(17)-\mathrm{C}(18)-\mathrm{H}(18)$ & 120.0 \\
\hline$C(9)-C(8)-C(17)$ & $115(6)$ & $\mathrm{C}(19)-\mathrm{C}(18)-\mathrm{H}(18)$ & 120.0 \\
\hline $\mathrm{N}(3)-\mathrm{C}(9)-\mathrm{C}(8)$ & $123(6)$ & $\mathrm{C}(18)-\mathrm{C}(19)-\mathrm{C}(20)$ & 120.0 \\
\hline $\mathrm{N}(3)-\mathrm{C}(9)-\mathrm{C}(10)$ & $110(6)$ & $\mathrm{C}(18)-\mathrm{C}(19)-\mathrm{H}(19)$ & 120.0 \\
\hline $\mathrm{C}(8)-\mathrm{C}(9)-\mathrm{C}(10)$ & $125(5)$ & $\mathrm{C}(20)-\mathrm{C}(19)-\mathrm{H}(19)$ & 120.0 \\
\hline $\mathrm{C}(10) \# 1-\mathrm{C}(10)-\mathrm{C}(9)$ & $107(3)$ & $\mathrm{C}(19)-\mathrm{C}(20)-\mathrm{N}(5)$ & 120.0 \\
\hline $\mathrm{C}(10) \# 1-\mathrm{C}(10)-\mathrm{H}(10)$ & 126.6 & $\mathrm{C}(19)-\mathrm{C}(20)-\mathrm{H}(20)$ & 120.0 \\
\hline $\mathrm{C}(9)-\mathrm{C}(10)-\mathrm{H}(10)$ & 126.6 & $\mathrm{~N}(5)-\mathrm{C}(20)-\mathrm{H}(20)$ & 120.0 \\
\hline$C(11)-N(4)-C(15)$ & $118(4)$ & $\mathrm{N}(5) \# 2-\mathrm{Pt}(2)-\mathrm{N}(5)$ & $91.8(19)$ \\
\hline $\mathrm{C}(11)-\mathrm{N}(4)-\mathrm{Pt}(1)$ & $125(4)$ & $\mathrm{N}(5) \# 2-\mathrm{Pt}(2)-\mathrm{N}(11) \# 2$ & $175.9(16)$ \\
\hline $\mathrm{C}(15)-\mathrm{N}(4)-\mathrm{Pt}(1)$ & $117(3)$ & $\mathrm{N}(5)-\mathrm{Pt}(2)-\mathrm{N}(11) \# 2$ & $92.2(15)$ \\
\hline $\mathrm{N}(4)-\mathrm{C}(11)-\mathrm{C}(12)$ & $119(5)$ & $\mathrm{N}(5) \# 2-\mathrm{Pt}(2)-\mathrm{N}(11)$ & $92.2(15)$ \\
\hline $\mathrm{N}(4)-\mathrm{C}(11)-\mathrm{H}(11)$ & 120.4 & $\mathrm{~N}(5)-\operatorname{Pt}(2)-\mathrm{N}(11)$ & $175.9(16)$ \\
\hline $\mathrm{C}(12)-\mathrm{C}(11)-\mathrm{H}(11)$ & 120.4 & $\mathrm{~N}(11) \# 2-\mathrm{Pt}(2)-\mathrm{N}(11)$ & $84(2)$ \\
\hline $\mathrm{C}(13)-\mathrm{C}(12)-\mathrm{C}(11)$ & $129(6)$ & $\mathrm{C}(37)-\mathrm{N}(11)-\mathrm{C}(39)$ & $116(3)$ \\
\hline $\mathrm{C}(13)-\mathrm{C}(12)-\mathrm{C}(3)$ & $110(5)$ & $\mathrm{C}(37)-\mathrm{N}(11)-\mathrm{C}(38)$ & $100(3)$ \\
\hline $\mathrm{C}(11)-\mathrm{C}(12)-\mathrm{C}(3)$ & $121(5)$ & $\mathrm{C}(39)-\mathrm{N}(11)-\mathrm{C}(38)$ & $103(3)$ \\
\hline $\mathrm{C}(12)-\mathrm{C}(13)-\mathrm{C}(14)$ & $122(5)$ & $\mathrm{C}(37)-\mathrm{N}(11)-\mathrm{Pt}(2)$ & $114(3)$ \\
\hline $\mathrm{C}(12)-\mathrm{C}(13)-\mathrm{H}(13)$ & 119.2 & $\mathrm{C}(39)-\mathrm{N}(11)-\mathrm{Pt}(2)$ & $111(3)$ \\
\hline $\mathrm{C}(14)-\mathrm{C}(13)-\mathrm{H}(13)$ & 119.2 & $\mathrm{C}(38)-\mathrm{N}(11)-\mathrm{Pt}(2)$ & $112(2)$ \\
\hline$C(13)-C(14)-C(15)$ & $105(4)$ & $\mathrm{N}(11)-\mathrm{C}(37)-\mathrm{H}(37 \mathrm{~A})$ & 109.5 \\
\hline $\mathrm{C}(13)-\mathrm{C}(14)-\mathrm{H}(14)$ & 127.6 & $\mathrm{~N}(11)-\mathrm{C}(37)-\mathrm{H}(37 \mathrm{~B})$ & 109.5 \\
\hline $\mathrm{C}(15)-\mathrm{C}(14)-\mathrm{H}(14)$ & 127.6 & $\mathrm{H}(37 \mathrm{~A})-\mathrm{C}(37)-\mathrm{H}(37 \mathrm{~B})$ & 109.5 \\
\hline $\mathrm{N}(4)-\mathrm{C}(15)-\mathrm{C}(14)$ & $127(4)$ & $\mathrm{N}(11)-\mathrm{C}(37)-\mathrm{H}(37 \mathrm{C})$ & 109.5 \\
\hline $\mathrm{N}(4)-\mathrm{C}(15)-\mathrm{H}(15)$ & 116.6 & $\mathrm{H}(37 \mathrm{~A})-\mathrm{C}(37)-\mathrm{H}(37 \mathrm{C})$ & 109.5 \\
\hline $\mathrm{C}(14)-\mathrm{C}(15)-\mathrm{H}(15)$ & 116.6 & H(37B)-C(37)-H(37C) & 109.5 \\
\hline$C(16)-N(5)-C(20)$ & 120.0 & $\mathrm{~N}(11)-\mathrm{C}(38)-\mathrm{H}(38 \mathrm{~A})$ & 109.5 \\
\hline $\mathrm{C}(16)-\mathrm{N}(5)-\mathrm{Pt}(2)$ & $115(3)$ & $\mathrm{N}(11)-\mathrm{C}(38)-\mathrm{H}(38 \mathrm{~B})$ & 109.5 \\
\hline $\mathrm{C}(20)-\mathrm{N}(5)-\mathrm{Pt}(2)$ & $125(3)$ & $\mathrm{H}(38 \mathrm{~A})-\mathrm{C}(38)-\mathrm{H}(38 \mathrm{~B})$ & 109.5 \\
\hline $\mathrm{N}(5)-\mathrm{C}(16)-\mathrm{C}(17)$ & 120.0 & $\mathrm{~N}(11)-\mathrm{C}(38)-\mathrm{H}(38 \mathrm{C})$ & 109.5 \\
\hline $\mathrm{N}(5)-\mathrm{C}(16)-\mathrm{H}(16)$ & 120.0 & $\mathrm{H}(38 \mathrm{~A})-\mathrm{C}(38)-\mathrm{H}(38 \mathrm{C})$ & 109.5 \\
\hline $\mathrm{C}(17)-\mathrm{C}(16)-\mathrm{H}(16)$ & 120.0 & $\mathrm{H}(38 \mathrm{~B})-\mathrm{C}(38)-\mathrm{H}(38 \mathrm{C})$ & 109.5 \\
\hline $\mathrm{C}(18)-\mathrm{C}(17)-\mathrm{C}(16)$ & 120.0 & $\mathrm{~N}(11)-\mathrm{C}(39)-\mathrm{C}(39) \# 2$ & $113(2)$ \\
\hline $\mathrm{C}(18)-\mathrm{C}(17)-\mathrm{C}(8)$ & $117(4)$ & $\mathrm{N}(11)-\mathrm{C}(39)-\mathrm{H}(39 \mathrm{~A})$ & 109.0 \\
\hline $\mathrm{C}(16)-\mathrm{C}(17)-\mathrm{C}(8)$ & $123(4)$ & $\mathrm{C}(39) \# 2-\mathrm{C}(39)-\mathrm{H}(39 \mathrm{~A})$ & 109.0 \\
\hline
\end{tabular}




\begin{tabular}{|c|c|c|c|}
\hline N(11)-C(39)-H(39B) & 109.0 & $\mathrm{C}(34)-\mathrm{C}(35)-\mathrm{H}(35)$ & 124.6 \\
\hline $\mathrm{C}(39) \# 2-\mathrm{C}(39)-\mathrm{H}(39 \mathrm{~B})$ & 109.0 & $\mathrm{~N}(10)-\mathrm{C}(36)-\mathrm{C}(35)$ & $125(4)$ \\
\hline H(39A)-C(39)-H(39B) & 107.8 & $\mathrm{~N}(10)-\mathrm{C}(36)-\mathrm{H}(36)$ & 117.6 \\
\hline $\mathrm{C}(28)-\mathrm{N}(8)-\mathrm{C}(28) \# 1$ & $101(5)$ & $\mathrm{C}(35)-\mathrm{C}(36)-\mathrm{H}(36)$ & 117.6 \\
\hline $\mathrm{C}(30) \# 2-\mathrm{N}(9)-\mathrm{C}(30)$ & $119(6)$ & $\mathrm{F}(3)-\mathrm{P}(1)-\mathrm{F}(4)$ & $87.0(13)$ \\
\hline $\mathrm{C}(30) \# 2-\mathrm{N}(9)-\mathrm{H}(9 \mathrm{~N})$ & 120.5 & $F(3)-P(1)-F(6)$ & $86.8(11)$ \\
\hline $\mathrm{C}(30)-\mathrm{N}(9)-\mathrm{H}(9 \mathrm{~N})$ & 120.5 & $\mathrm{~F}(4)-\mathrm{P}(1)-\mathrm{F}(6)$ & $89.7(11)$ \\
\hline $\mathrm{C}(28)-\mathrm{C}(27)-\mathrm{C}(27) \# 1$ & $103(3)$ & $\mathrm{F}(3)-\mathrm{P}(1)-\mathrm{F}(1)$ & $177.3(10)$ \\
\hline $\mathrm{C}(28)-\mathrm{C}(27)-\mathrm{H}(27)$ & 128.4 & $F(4)-P(1)-F(1)$ & $91.2(7)$ \\
\hline $\mathrm{C}(27) \# 1-\mathrm{C}(27)-\mathrm{H}(27)$ & 128.4 & $\mathrm{~F}(6)-\mathrm{P}(1)-\mathrm{F}(1)$ & $91.1(6)$ \\
\hline $\mathrm{C}(27)-\mathrm{C}(28)-\mathrm{N}(8)$ & $116(5)$ & $F(3)-P(1)-F(2)$ & $91.1(6)$ \\
\hline $\mathrm{C}(27)-\mathrm{C}(28)-\mathrm{C}(29)$ & $117(4)$ & $\mathrm{F}(4)-\mathrm{P}(1)-\mathrm{F}(2)$ & $178.1(10)$ \\
\hline $\mathrm{N}(8)-\mathrm{C}(28)-\mathrm{C}(29)$ & $126(4)$ & $F(6)-P(1)-F(2)$ & $90.5(6)$ \\
\hline $\mathrm{C}(30)-\mathrm{C}(29)-\mathrm{C}(33)$ & $114(4)$ & $\mathrm{F}(1)-\mathrm{P}(1)-\mathrm{F}(2)$ & $90.7(6)$ \\
\hline$C(30)-C(29)-C(28)$ & $120(5)$ & $\mathrm{F}(3)-\mathrm{P}(1)-\mathrm{F}(5)$ & $91.8(11)$ \\
\hline $\mathrm{C}(33)-\mathrm{C}(29)-\mathrm{C}(28)$ & $126(4)$ & $\mathrm{F}(4)-\mathrm{P}(1)-\mathrm{F}(5)$ & $89.9(11)$ \\
\hline $\mathrm{C}(29)-\mathrm{C}(30)-\mathrm{N}(9)$ & $132(5)$ & $\mathrm{F}(6)-\mathrm{P}(1)-\mathrm{F}(5)$ & $178.6(10)$ \\
\hline $\mathrm{C}(29)-\mathrm{C}(30)-\mathrm{C}(31)$ & $127(5)$ & $\mathrm{F}(1)-\mathrm{P}(1)-\mathrm{F}(5)$ & $90.2(6)$ \\
\hline $\mathrm{N}(9)-\mathrm{C}(30)-\mathrm{C}(31)$ & $100(5)$ & $\mathrm{F}(2)-\mathrm{P}(1)-\mathrm{F}(5)$ & $89.9(6)$ \\
\hline $\mathrm{C}(31) \# 2-\mathrm{C}(31)-\mathrm{C}(30)$ & $110(3)$ & $\mathrm{F}(8 \mathrm{~B})-\mathrm{P}(2)-\mathrm{F}(7 \mathrm{~B})$ & $87.3(15)$ \\
\hline $\mathrm{C}(31) \# 2-\mathrm{C}(31)-\mathrm{H}(31)$ & 125.0 & $\mathrm{~F}(8 \mathrm{~B})-\mathrm{P}(2)-\mathrm{F}(10)$ & $87.2(11)$ \\
\hline $\mathrm{C}(30)-\mathrm{C}(31)-\mathrm{H}(31)$ & 125.0 & $\mathrm{~F}(7 \mathrm{~B})-\mathrm{P}(2)-\mathrm{F}(10)$ & $90.3(12)$ \\
\hline $\mathrm{C}(36)-\mathrm{N}(10)-\mathrm{C}(32)$ & $122(4)$ & $\mathrm{F}(8 \mathrm{~B})-\mathrm{P}(2)-\mathrm{F}(7 \mathrm{~A})$ & $178.1(14)$ \\
\hline $\mathrm{C}(36)-\mathrm{N}(10)-\mathrm{Pt}(1)$ & $124(3)$ & $\mathrm{F}(7 \mathrm{~B})-\mathrm{P}(2)-\mathrm{F}(7 \mathrm{~A})$ & $91.3(12)$ \\
\hline $\mathrm{C}(32)-\mathrm{N}(10)-\mathrm{Pt}(1)$ & $114(3)$ & $F(10)-P(2)-F(7 A)$ & $91.6(9)$ \\
\hline $\mathrm{C}(33)-\mathrm{C}(32)-\mathrm{N}(10)$ & $117(4)$ & $\mathrm{F}(8 \mathrm{~B})-\mathrm{P}(2)-\mathrm{F}(9)$ & $92.3(11)$ \\
\hline $\mathrm{C}(33)-\mathrm{C}(32)-\mathrm{H}(32 \mathrm{~A})$ & 121.5 & $\mathrm{~F}(7 \mathrm{~B})-\mathrm{P}(2)-\mathrm{F}(9)$ & $90.5(12)$ \\
\hline $\mathrm{N}(10)-\mathrm{C}(32)-\mathrm{H}(32 \mathrm{~A})$ & 121.5 & $\mathrm{~F}(10)-\mathrm{P}(2)-\mathrm{F}(9)$ & $179.0(14)$ \\
\hline $\mathrm{C}(32)-\mathrm{C}(33)-\mathrm{C}(34)$ & $119(5)$ & $\mathrm{F}(7 \mathrm{~A})-\mathrm{P}(2)-\mathrm{F}(9)$ & $88.9(9)$ \\
\hline $\mathrm{C}(32)-\mathrm{C}(33)-\mathrm{C}(29)$ & $122(5)$ & $\mathrm{F}(8 \mathrm{~B})-\mathrm{P}(2)-\mathrm{F}(8 \mathrm{~A})$ & $91.0(12)$ \\
\hline $\mathrm{C}(34)-\mathrm{C}(33)-\mathrm{C}(29)$ & $119(5)$ & $\mathrm{F}(7 \mathrm{~B})-\mathrm{P}(2)-\mathrm{F}(8 \mathrm{~A})$ & $178.2(14)$ \\
\hline $\mathrm{C}(33)-\mathrm{C}(34)-\mathrm{C}(35)$ & $126(5)$ & $\mathrm{F}(10)-\mathrm{P}(2)-\mathrm{F}(8 \mathrm{~A})$ & $90.3(10)$ \\
\hline $\mathrm{C}(33)-\mathrm{C}(34)-\mathrm{H}(34)$ & 117.1 & $\mathrm{~F}(7 \mathrm{~A})-\mathrm{P}(2)-\mathrm{F}(8 \mathrm{~A})$ & $90.5(11)$ \\
\hline $\mathrm{C}(35)-\mathrm{C}(34)-\mathrm{H}(34)$ & 117.1 & $\mathrm{~F}(9)-\mathrm{P}(2)-\mathrm{F}(8 \mathrm{~A})$ & $88.9(10)$ \\
\hline $\mathrm{C}(36)-\mathrm{C}(35)-\mathrm{C}(34)$ & $111(5)$ & $\mathrm{F}(11 \mathrm{~B})-\mathrm{P}(3)-\mathrm{F}(12 \mathrm{~B})$ & $87.0(15)$ \\
\hline $\mathrm{C}(36)-\mathrm{C}(35)-\mathrm{H}(35)$ & 124.6 & $\mathrm{~F}(11 \mathrm{~B})-\mathrm{P}(3)-\mathrm{F}(13 \mathrm{~B})$ & $87.0(12)$ \\
\hline
\end{tabular}




\begin{tabular}{|c|c|c|c|}
\hline $\mathrm{F}(12 \mathrm{~B})-\mathrm{P}(3)-\mathrm{F}(13 \mathrm{~B})$ & $90.0(13)$ & $\mathrm{F}(18 \mathrm{~B})-\mathrm{P}(4)-\mathrm{F}(15)$ & $90.1(10)$ \\
\hline F(11B)-P(3)-F(11A) & $178.2(14)$ & $\mathrm{F}(18 \mathrm{~B}) \# 2-\mathrm{P}(4)-\mathrm{F}(15)$ & $90.1(10)$ \\
\hline F(12B)-P(3)-F(11A) & $91.5(12)$ & $F(14)-P(4)-F(15)$ & $90.3(11)$ \\
\hline F(13B)-P(3)-F(11A) & $91.9(11)$ & $\mathrm{F}(18 \mathrm{~A}) \# 2-\mathrm{P}(4)-\mathrm{F}(15)$ & $89.5(10)$ \\
\hline $\mathrm{F}(11 \mathrm{~B})-\mathrm{P}(3)-\mathrm{F}(13 \mathrm{~A})$ & $92.2(12)$ & $\mathrm{F}(18 \mathrm{~A})-\mathrm{P}(4)-\mathrm{F}(15)$ & $89.5(10)$ \\
\hline $\mathrm{F}(12 \mathrm{~B})-\mathrm{P}(3)-\mathrm{F}(13 \mathrm{~A})$ & $90.5(12)$ & $\mathrm{F}(18 \mathrm{~B}) \# 2-\mathrm{F}(18 \mathrm{~A})-\mathrm{P}(4)$ & $75(10)$ \\
\hline F(13B)-P(3)-F(13A) & 179.1(14) & $\mathrm{F}(18 \mathrm{~A}) \# 2-\mathrm{F}(18 \mathrm{~B})-\mathrm{P}(4)$ & 101(10) \\
\hline $\mathrm{F}(11 \mathrm{~A})-\mathrm{P}(3)-\mathrm{F}(13 \mathrm{~A})$ & $88.9(10)$ & $\mathrm{N}(27)-\mathrm{C}(271)-\mathrm{C}(272)$ & $176(7)$ \\
\hline $\mathrm{F}(11 \mathrm{~B})-\mathrm{P}(3)-\mathrm{F}(12 \mathrm{~A})$ & $91.2(12)$ & $\mathrm{C}(271)-\mathrm{C}(272)-\mathrm{H}(271)$ & 100.7 \\
\hline $\mathrm{F}(12 \mathrm{~B})-\mathrm{P}(3)-\mathrm{F}(12 \mathrm{~A})$ & $178.1(15)$ & $\mathrm{C}(272) \# 3-\mathrm{C}(272)-\mathrm{H}(271)$ & 72.1 \\
\hline $\mathrm{F}(13 \mathrm{~B})-\mathrm{P}(3)-\mathrm{F}(12 \mathrm{~A})$ & $90.4(11)$ & $\mathrm{C}(271)-\mathrm{C}(272)-\mathrm{H}(272)$ & 102.1 \\
\hline $\mathrm{F}(11 \mathrm{~A})-\mathrm{P}(3)-\mathrm{F}(12 \mathrm{~A})$ & $90.3(11)$ & $\mathrm{C}(272) \# 3-\mathrm{C}(272)-\mathrm{H}(272)$ & 72.1 \\
\hline $\mathrm{F}(13 \mathrm{~A})-\mathrm{P}(3)-\mathrm{F}(12 \mathrm{~A})$ & $89.1(10)$ & $\mathrm{H}(271)-\mathrm{C}(272)-\mathrm{H}(272)$ & 88.9 \\
\hline$F(16)-P(4)-F(17)$ & $87.2(15)$ & $\mathrm{C}(271)-\mathrm{C}(272)-\mathrm{H}(273)$ & 120.6 \\
\hline $\mathrm{F}(16)-\mathrm{P}(4)-\mathrm{F}(18 \mathrm{~B})$ & $87.7(10)$ & $\mathrm{C}(272) \# 3-\mathrm{C}(272)-\mathrm{H}(273)$ & 68.8 \\
\hline $\mathrm{F}(17)-\mathrm{P}(4)-\mathrm{F}(18 \mathrm{~B})$ & $89.8(10)$ & $\mathrm{H}(271)-\mathrm{C}(272)-\mathrm{H}(273)$ & 116.3 \\
\hline $\mathrm{F}(16)-\mathrm{P}(4)-\mathrm{F}(18 \mathrm{~B}) \# 2$ & $87.7(10)$ & $\mathrm{H}(272)-\mathrm{C}(272)-\mathrm{H}(273)$ & 122.2 \\
\hline $\mathrm{F}(17)-\mathrm{P}(4)-\mathrm{F}(18 \mathrm{~B}) \# 2$ & $89.8(10)$ & $\mathrm{F}(21)-\mathrm{P}(5)-\mathrm{F}(22)$ & $86.9(15)$ \\
\hline $\mathrm{F}(18 \mathrm{~B})-\mathrm{P}(4)-\mathrm{F}(18 \mathrm{~B}) \# 2$ & $175(2)$ & $\mathrm{F}(21)-\mathrm{P}(5)-\mathrm{F}(24)$ & $87.3(12)$ \\
\hline $\mathrm{F}(16)-\mathrm{P}(4)-\mathrm{F}(14)$ & $178.9(15)$ & $\mathrm{F}(22)-\mathrm{P}(5)-\mathrm{F}(24)$ & $90.3(13)$ \\
\hline$F(17)-P(4)-F(14)$ & $91.6(12)$ & $\mathrm{F}(21)-\mathrm{P}(5)-\mathrm{F}(19)$ & $178.3(15)$ \\
\hline F(18B)-P(4)-F(14) & $92.3(10)$ & $\mathrm{F}(22)-\mathrm{P}(5)-\mathrm{F}(19)$ & $91.5(12)$ \\
\hline $\mathrm{F}(18 \mathrm{~B}) \# 2-\mathrm{P}(4)-\mathrm{F}(14)$ & $92.3(10)$ & $\mathrm{F}(24)-\mathrm{P}(5)-\mathrm{F}(19)$ & $91.9(10)$ \\
\hline $\mathrm{F}(16)-\mathrm{P}(4)-\mathrm{F}(18 \mathrm{~A}) \# 2$ & $91.4(9)$ & $\mathrm{F}(21)-\mathrm{P}(5)-\mathrm{F}(20)$ & 91.1(12) \\
\hline $\mathrm{F}(17)-\mathrm{P}(4)-\mathrm{F}(18 \mathrm{~A}) \# 2$ & $90.5(10)$ & $\mathrm{F}(22)-\mathrm{P}(5)-\mathrm{F}(20)$ & $177.9(15)$ \\
\hline $\mathrm{F}(18 \mathrm{~B})-\mathrm{P}(4)-\mathrm{F}(18 \mathrm{~A}) \# 2$ & $3.7(15)$ & $\mathrm{F}(24)-\mathrm{P}(5)-\mathrm{F}(20)$ & $90.5(11)$ \\
\hline $\mathrm{F}(18 \mathrm{~B}) \# 2-\mathrm{P}(4)-\mathrm{F}(18 \mathrm{~A}) \# 2$ & $179.0(14)$ & $\mathrm{F}(19)-\mathrm{P}(5)-\mathrm{F}(20)$ & $90.4(11)$ \\
\hline $\mathrm{F}(14)-\mathrm{P}(4)-\mathrm{F}(18 \mathrm{~A}) \# 2$ & $88.6(9)$ & $\mathrm{F}(21)-\mathrm{P}(5)-\mathrm{F}(23)$ & $91.8(12)$ \\
\hline $\mathrm{F}(16)-\mathrm{P}(4)-\mathrm{F}(18 \mathrm{~A})$ & $91.4(9)$ & $\mathrm{F}(22)-\mathrm{P}(5)-\mathrm{F}(23)$ & $90.1(12)$ \\
\hline $\mathrm{F}(17)-\mathrm{P}(4)-\mathrm{F}(18 \mathrm{~A})$ & $90.5(10)$ & $\mathrm{F}(24)-\mathrm{P}(5)-\mathrm{F}(23)$ & $178.9(14)$ \\
\hline $\mathrm{F}(18 \mathrm{~B})-\mathrm{P}(4)-\mathrm{F}(18 \mathrm{~A})$ & 179.1(14) & $\mathrm{F}(19)-\mathrm{P}(5)-\mathrm{F}(23)$ & $89.1(10)$ \\
\hline F(18B)\#2-P(4)-F(18A) & $3.7(15)$ & $\mathrm{F}(20)-\mathrm{P}(5)-\mathrm{F}(23)$ & 89.1(11) \\
\hline $\mathrm{F}(14)-\mathrm{P}(4)-\mathrm{F}(18 \mathrm{~A})$ & $88.6(9)$ & $\mathrm{N}(21)-\mathrm{C}(211)-\mathrm{C}(212)$ & $176(6)$ \\
\hline $\mathrm{F}(18 \mathrm{~A}) \# 2-\mathrm{P}(4)-\mathrm{F}(18 \mathrm{~A})$ & $177.0(19)$ & $\mathrm{C}(211)-\mathrm{C}(212)-\mathrm{H}(211)$ & 109.6 \\
\hline $\mathrm{F}(16)-\mathrm{P}(4)-\mathrm{F}(15)$ & $90.8(12)$ & $\mathrm{C}(211)-\mathrm{C}(212)-\mathrm{H}(212)$ & 124.5 \\
\hline $\mathrm{F}(17)-\mathrm{P}(4)-\mathrm{F}(15)$ & $178.0(15)$ & $\mathrm{H}(211)-\mathrm{C}(212)-\mathrm{H}(212)$ & 101.2 \\
\hline
\end{tabular}




\begin{tabular}{|c|c|c|c|}
\hline $\mathrm{C}(211)-\mathrm{C}(212)-\mathrm{H}(213)$ & 115.0 & $\mathrm{H}(232)-\mathrm{C}(232)-\mathrm{H}(233) \# 1$ & 82.9 \\
\hline $\mathrm{H}(211)-\mathrm{C}(212)-\mathrm{H}(213)$ & 94.6 & $\mathrm{H}(233)-\mathrm{C}(232)-\mathrm{H}(233) \# 1$ & 34.6 \\
\hline $\mathrm{H}(212)-\mathrm{C}(212)-\mathrm{H}(213)$ & 107.0 & $\mathrm{H}(231) \# 1-\mathrm{C}(232)-\mathrm{H}(233) \# 1$ & 111.1 \\
\hline $\mathrm{C}(211)-\mathrm{C}(212)-\mathrm{H}(212) \# 2$ & $124(7)$ & $\mathrm{H}(232) \# 1-\mathrm{C}(232)-\mathrm{H}(233) \# 1$ & 114.5 \\
\hline $\mathrm{H}(211)-\mathrm{C}(212)-\mathrm{H}(212) \# 2$ & 125.9 & $\mathrm{~N}(24)-\mathrm{C}(241)-\mathrm{C}(242)$ & $176(6)$ \\
\hline $\mathrm{H}(212)-\mathrm{C}(212)-\mathrm{H}(212) \# 2$ & 49.9 & $\mathrm{~N}(24)-\mathrm{C}(241)-\mathrm{H}(243)$ & 149.3 \\
\hline $\mathrm{H}(213)-\mathrm{C}(212)-\mathrm{H}(212) \# 2$ & 63.4 & $\mathrm{C}(242)-\mathrm{C}(241)-\mathrm{H}(243)$ & 33.3 \\
\hline $\mathrm{N}(22)-\mathrm{C}(221)-\mathrm{C}(222)$ & $178(10)$ & $\mathrm{C}(241)-\mathrm{C}(242)-\mathrm{H}(241)$ & 94.7 \\
\hline $\mathrm{C}(221)-\mathrm{C}(222)-\mathrm{H}(221)$ & 120.5 & $\mathrm{C}(241)-\mathrm{C}(242)-\mathrm{H}(242)$ & 99.0 \\
\hline $\mathrm{C}(221)-\mathrm{C}(222)-\mathrm{H}(222)$ & 117.4 & $\mathrm{H}(241)-\mathrm{C}(242)-\mathrm{H}(242)$ & 112.4 \\
\hline $\mathrm{H}(221)-\mathrm{C}(222)-\mathrm{H}(222)$ & 102.4 & $\mathrm{C}(241)-\mathrm{C}(242)-\mathrm{H}(243)$ & 80.6 \\
\hline $\mathrm{C}(221)-\mathrm{C}(222)-\mathrm{H}(223)$ & 115.2 & $\mathrm{H}(241)-\mathrm{C}(242)-\mathrm{H}(243)$ & 136.5 \\
\hline $\mathrm{H}(221)-\mathrm{C}(222)-\mathrm{H}(223)$ & 100.1 & $\mathrm{H}(242)-\mathrm{C}(242)-\mathrm{H}(243)$ & 111.0 \\
\hline $\mathrm{H}(222)-\mathrm{C}(222)-\mathrm{H}(223)$ & 97.4 & $\mathrm{~N}(25)-\mathrm{C}(251)-\mathrm{C}(252)$ & $177(9)$ \\
\hline $\mathrm{C}(221)-\mathrm{C}(222)-\mathrm{H}(221) \# 2$ & $120(9)$ & $\mathrm{C}(251)-\mathrm{C}(252)-\mathrm{H}(251)$ & 116.1 \\
\hline $\mathrm{H}(221)-\mathrm{C}(222)-\mathrm{H}(221) \# 2$ & 47.8 & $\mathrm{C}(251)-\mathrm{C}(252)-\mathrm{H}(252)$ & 128.6 \\
\hline $\mathrm{H}(222)-\mathrm{C}(222)-\mathrm{H}(221) \# 2$ & 60.2 & $\mathrm{H}(251)-\mathrm{C}(252)-\mathrm{H}(252)$ & 86.3 \\
\hline $\mathrm{H}(223)-\mathrm{C}(222)-\mathrm{H}(221) \# 2$ & 124.2 & $\mathrm{C}(251)-\mathrm{C}(252)-\mathrm{H}(253)$ & 129.1 \\
\hline $\mathrm{N}(23)-\mathrm{C}(231)-\mathrm{C}(232)$ & $176(6)$ & $\mathrm{H}(251)-\mathrm{C}(252)-\mathrm{H}(253)$ & 88.2 \\
\hline $\mathrm{C}(231)-\mathrm{C}(232)-\mathrm{H}(231)$ & 107.6 & $\mathrm{H}(252)-\mathrm{C}(252)-\mathrm{H}(253)$ & 94.9 \\
\hline $\mathrm{C}(231)-\mathrm{C}(232)-\mathrm{H}(232)$ & 108.4 & $\mathrm{~N}(26)-\mathrm{C}(261)-\mathrm{C}(262)$ & $177(8)$ \\
\hline $\mathrm{H}(231)-\mathrm{C}(232)-\mathrm{H}(232)$ & 104.0 & $\mathrm{C}(261)-\mathrm{C}(262)-\mathrm{H}(261)$ & 106.9 \\
\hline $\mathrm{C}(231)-\mathrm{C}(232)-\mathrm{H}(233)$ & 110.8 & $\mathrm{C}(261)-\mathrm{C}(262)-\mathrm{H}(262)$ & 103.5 \\
\hline $\mathrm{H}(231)-\mathrm{C}(232)-\mathrm{H}(233)$ & 111.1 & $\mathrm{H}(261)-\mathrm{C}(262)-\mathrm{H}(262)$ & 124.0 \\
\hline $\mathrm{H}(232)-\mathrm{C}(232)-\mathrm{H}(233)$ & 114.4 & $\mathrm{C}(261)-\mathrm{C}(262)-\mathrm{H}(263)$ & 101.1 \\
\hline $\mathrm{C}(231)-\mathrm{C}(232)-\mathrm{H}(231) \# 1$ & $108(6)$ & $\mathrm{H}(261)-\mathrm{C}(262)-\mathrm{H}(263)$ & 113.8 \\
\hline $\mathrm{H}(231)-\mathrm{C}(232)-\mathrm{H}(231) \# 1$ & 75.3 & $\mathrm{H}(262)-\mathrm{C}(262)-\mathrm{H}(263)$ & 104.8 \\
\hline $\mathrm{H}(232)-\mathrm{C}(232)-\mathrm{H}(231) \# 1$ & 30.7 & $\mathrm{C}(261)-\mathrm{C}(262)-\mathrm{H}(261) \# 2$ & $107(6)$ \\
\hline $\mathrm{H}(233)-\mathrm{C}(232)-\mathrm{H}(231) \# 1$ & 136.3 & $\mathrm{H}(261)-\mathrm{C}(262)-\mathrm{H}(261) \# 2$ & 63.5 \\
\hline $\mathrm{C}(231)-\mathrm{C}(232)-\mathrm{H}(232) \# 1$ & $108(4)$ & $\mathrm{H}(262)-\mathrm{C}(262)-\mathrm{H}(261) \# 2$ & 63.2 \\
\hline $\mathrm{H}(231)-\mathrm{C}(232)-\mathrm{H}(232) \# 1$ & 30.7 & $\mathrm{H}(263)-\mathrm{C}(262)-\mathrm{H}(261) \# 2$ & 151.4 \\
\hline $\mathrm{H}(232)-\mathrm{C}(232)-\mathrm{H}(232) \# 1$ & 129.1 & $\mathrm{C}(261)-\mathrm{C}(262)-\mathrm{H}(262) \# 2$ & 103(3) \\
\hline $\mathrm{H}(233)-\mathrm{C}(232)-\mathrm{H}(232) \# 1$ & 83.0 & $\mathrm{H}(261)-\mathrm{C}(262)-\mathrm{H}(262) \# 2$ & 63.2 \\
\hline $\mathrm{H}(231) \# 1-\mathrm{C}(232)-\mathrm{H}(232) \# 1$ & 104.0 & $\mathrm{H}(262)-\mathrm{C}(262)-\mathrm{H}(262) \# 2$ & 147.6 \\
\hline $\mathrm{C}(231)-\mathrm{C}(232)-\mathrm{H}(233) \# 1$ & $111(7)$ & $\mathrm{H}(263)-\mathrm{C}(262)-\mathrm{H}(262) \# 2$ & 52.3 \\
\hline $\mathrm{H}(231)-\mathrm{C}(232)-\mathrm{H}(233) \# 1$ & 136.3 & $\mathrm{H}(261) \# 2-\mathrm{C}(262)-\mathrm{H}(262) \# 2$ & 124.0 \\
\hline
\end{tabular}




$\begin{array}{llll}\mathrm{N}(28)-\mathrm{C}(281)-\mathrm{C}(282) & 174(4) & \mathrm{O}(31)-\mathrm{C}(311)-\mathrm{H}(311) & 109.0 \\ \mathrm{C}(281)-\mathrm{C}(282)-\mathrm{H}(281) & 106.8 & \mathrm{C}(312)-\mathrm{C}(311)-\mathrm{H}(312) & 109.0 \\ \mathrm{C}(281)-\mathrm{C}(282)-\mathrm{H}(282) & 105.0 & \mathrm{O}(31)-\mathrm{C}(311)-\mathrm{H}(312) & 109.0 \\ \mathrm{H}(281)-\mathrm{C}(282)-\mathrm{H}(282) & 112.2 & \mathrm{H}(311)-\mathrm{C}(311)-\mathrm{H}(312) & 107.8 \\ \mathrm{C}(281)-\mathrm{C}(282)-\mathrm{H}(283) & 105.1 & \mathrm{C}(311)-\mathrm{C}(312)-\mathrm{H}(313) & 109.5 \\ \mathrm{H}(281)-\mathrm{C}(282)-\mathrm{H}(283) & 115.0 & \mathrm{C}(311)-\mathrm{C}(312)-\mathrm{H}(314) & 109.5 \\ \mathrm{H}(282)-\mathrm{C}(282)-\mathrm{H}(283) & 111.9 & \mathrm{H}(313)-\mathrm{C}(312)-\mathrm{H}(314) & 109.5 \\ \mathrm{C}(311) \# 3-\mathrm{O}(31)-\mathrm{C}(311) & 113.1(5) & \mathrm{C}(311)-\mathrm{C}(312)-\mathrm{H}(315) & 109.5 \\ \mathrm{C}(312)-\mathrm{C}(311)-\mathrm{O}(31) & 113.1(4) & \mathrm{H}(313)-\mathrm{C}(312)-\mathrm{H}(315) & 109.5 \\ \mathrm{C}(312)-\mathrm{C}(311)-\mathrm{H}(311) & 109.0 & \mathrm{H}(314)-\mathrm{C}(312)-\mathrm{H}(315) & 109.5\end{array}$

Symmetry transformations used to generate equivalent atoms:

$\# 1-\mathrm{x}+3 / 2, \mathrm{y}, \mathrm{z} \quad \# 2 \mathrm{x},-\mathrm{y}+3 / 2, \mathrm{z} \quad \# 3-\mathrm{x}+3 / 2,-\mathrm{y}+3 / 2, \mathrm{z}$

Table S4. Anisotropic displacement parameters $\left(\AA^{2} \times 10^{3}\right)$ for 1 a. The anisotropic displacement factor exponent takes the form: $-2 p^{2}\left[h^{2} a^{* 2} u^{11}+\ldots+2 h k a^{*} b^{*} u^{12}\right]$

\begin{tabular}{ccccccc}
\hline & $\mathrm{U}^{11}$ & $\mathrm{U}^{22}$ & $\mathrm{U}^{33}$ & $\mathrm{U}^{23}$ & $\mathrm{U}^{13}$ & $\mathrm{U}^{12}$ \\
\hline $\operatorname{Pt}(1)$ & $38(2)$ & $116(2)$ & $65(2)$ & $-2(2)$ & $1(1)$ & $-17(1)$ \\
$\operatorname{Pt}(2)$ & $69(6)$ & $104(11)$ & $67(6)$ & 0 & $54(4)$ & 0 \\
\hline
\end{tabular}




\section{Anion Binding Studies}

The association of $\mathrm{PF}_{6}{ }^{-}$(and $\mathrm{BF}_{4}^{-}$) inside of $\mathbf{1 a}, \mathbf{b}$ at $25^{\circ} \mathrm{C}$ in $\mathrm{CD}_{3} \mathrm{CN}$ was investigated by ${ }^{1} \mathrm{H}$ and ${ }^{19} \mathrm{~F}$ NMR spectroscopy. All ${ }^{1} \mathrm{H}$ NMR spectra were referenced to the residual proteo resonance of the solvent $\left({ }^{1} \mathrm{H} \delta 1.94 \mathrm{ppm}\right.$ for $\mathrm{CD}_{2} \mathrm{HCN}$ in $\left.\mathrm{CD}_{3} \mathrm{CN}\right)$, and ${ }^{19} \mathrm{~F}$ NMR spectra were referenced to a sealed capillary standard of fluorobenzene in $\mathrm{CD}_{3} \mathrm{CN}\left({ }^{19} \mathrm{~F} \delta-114.26 \mathrm{ppm}\right)$.

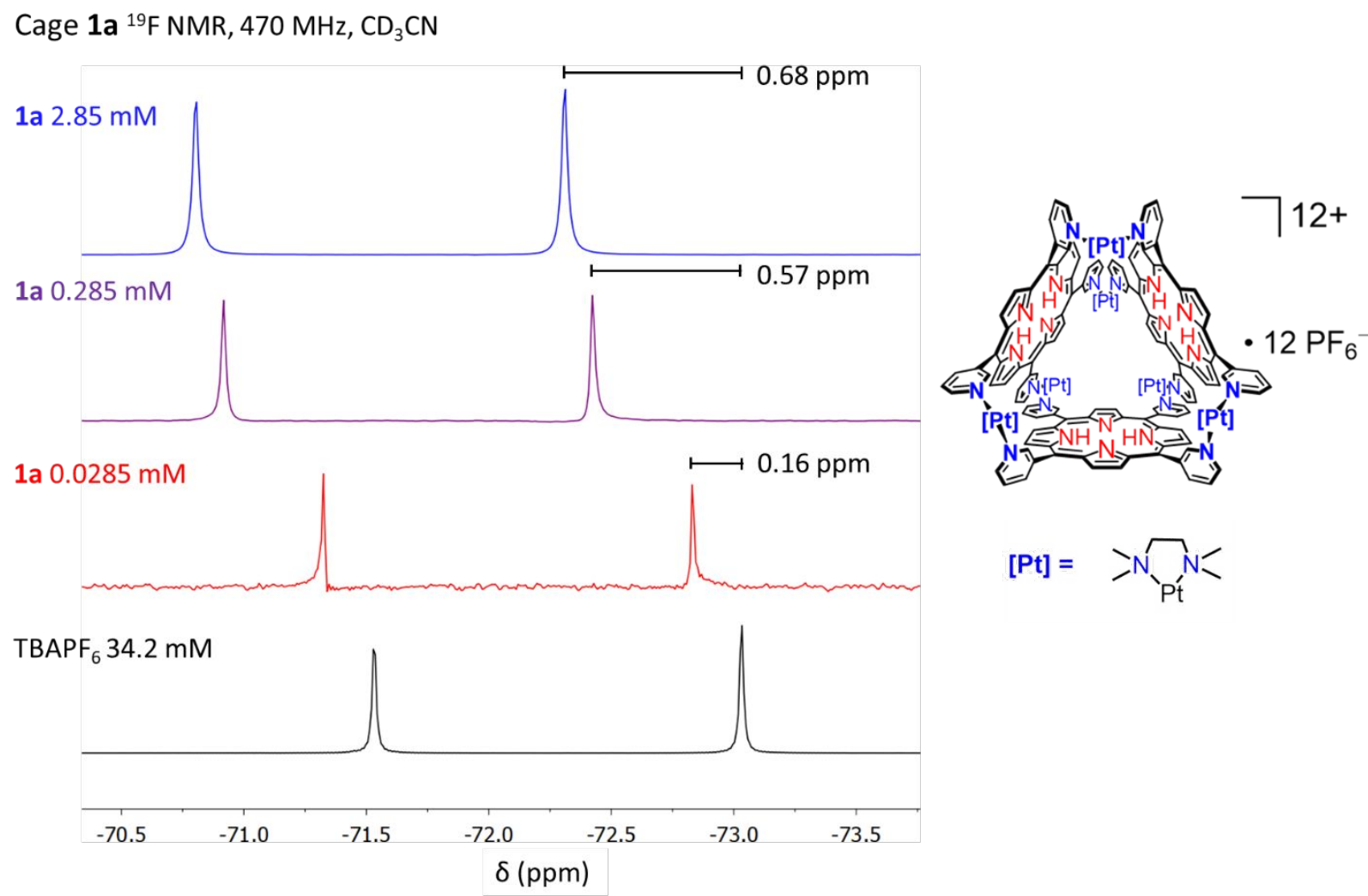

Figure S17: Stacked ${ }^{19} \mathrm{~F} N \mathrm{NMR}$ spectra $\left(\mathrm{CD}_{3} \mathrm{CN}\right)$ showing the shift of the $\mathrm{PF}_{6}{ }^{-}$resonance in a 2.85 $\mathrm{mM}$ sample of $1 \mathrm{a}$ upon 10 -fold and 100 -fold dilution. The ${ }^{19} \mathrm{~F}$ NMR spectrum of a $34.2 \mathrm{mM}$ sample of $\mathrm{TBAPF}_{6}$ in $\mathrm{CD}_{3} \mathrm{CN}$ is provided for comparison. The $\mathrm{PF}_{6}{ }^{-}$concentration in this latter sample matches that in the $2.85 \mathrm{mM}$ sample of $1 \mathrm{a}$. The TBAPF ${ }_{6}$ spectrum depicts the chemical shift of the unbound $\mathrm{PF}_{6}{ }^{-}$anions since $\left[\mathrm{NBu}_{4}\right]^{+}$cations do not interact strongly with these anions. Thus, differences between the spectra of $1 \mathbf{a}$ and that of $\mathrm{TBAPF}_{6}$ are indicative of the association of $\mathrm{PF}_{6}{ }^{-}$ with 1a. Dilution weakens the binding of the anions as evident from the ${ }^{19} \mathrm{~F}$ NMR resonance of $\mathrm{PF}_{6}{ }^{-}$shifting towards its unbound value upon dilution of a sample of $1 \mathrm{a}$. In control experiments, dilution of samples of $\mathrm{TBAPF}_{6}$ did not result in significant changes to the ${ }^{19} \mathrm{~F}$ NMR chemical shift of the $\mathrm{PF}_{6}{ }^{-}$resonance. 
Cage $1 \mathrm{a}{ }^{1} \mathrm{H}$ NMR, $500 \mathrm{MHz}, \mathrm{CD}_{3} \mathrm{CN}$

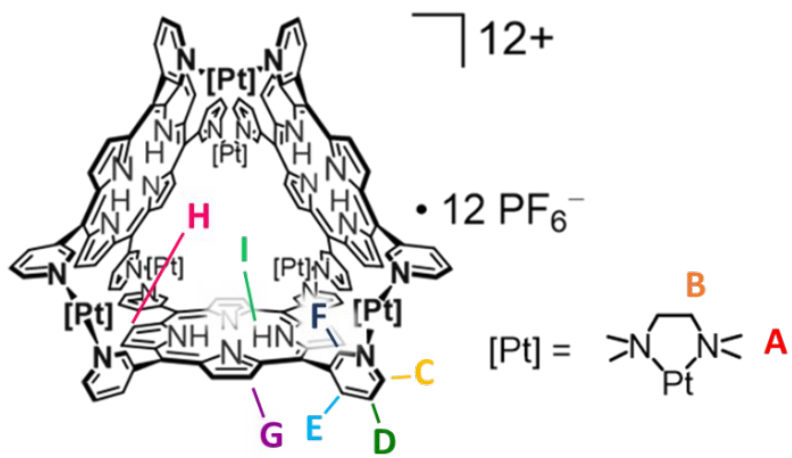

$1 \mathrm{a}$

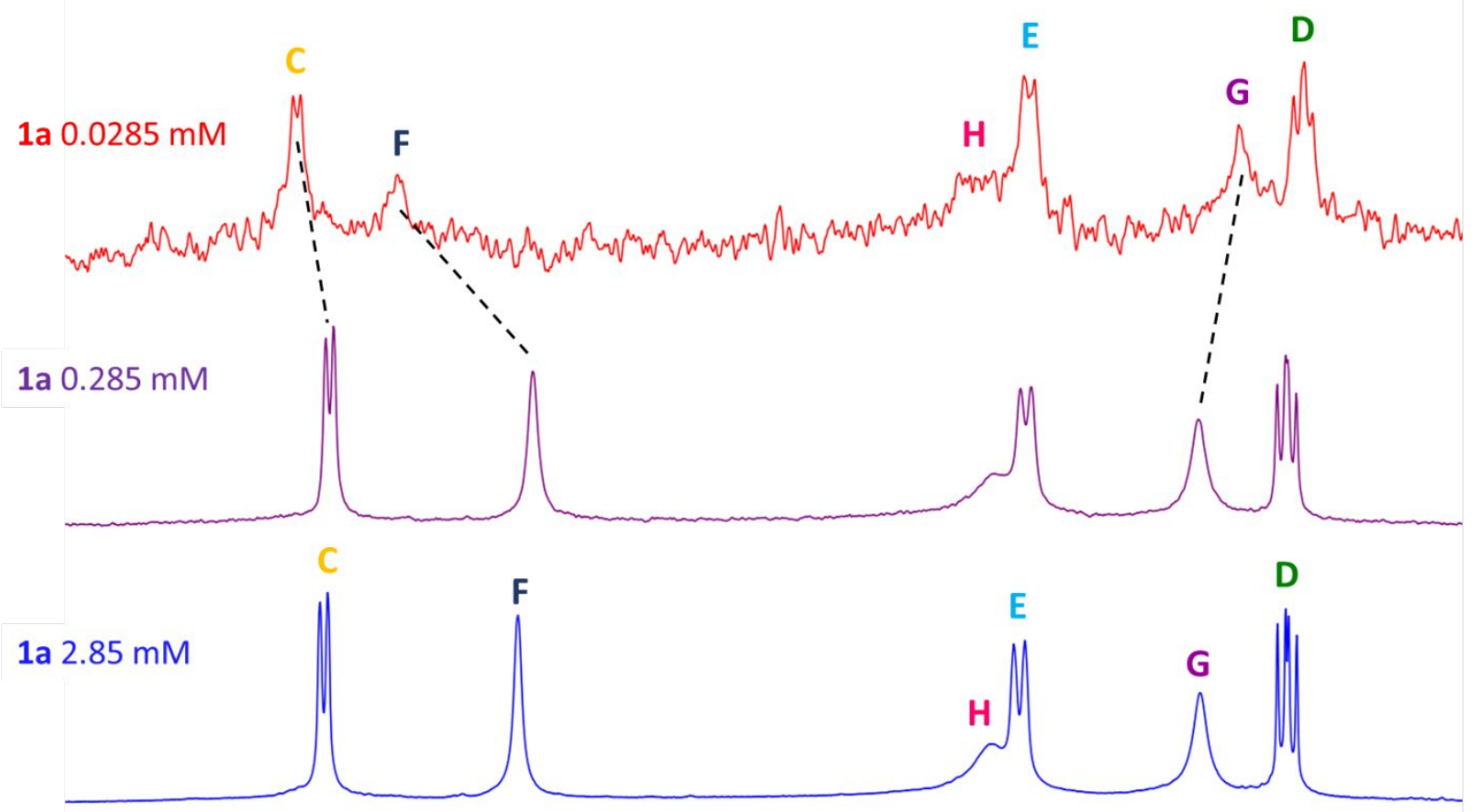

$\begin{array}{cccccccccccccccccccc}9.9 & 9.8 & 9.7 & 9.6 & 9.5 & 9.4 & 9.3 & 9.2 & 9.1 & 9.0 & 8.9 & 8.8 & 8.7 & 8.6 & 8.5 & 8.4 & 8.3 & 8.2 & 8.1 & 8.0\end{array}$

Figure S18: ${ }^{1} \mathrm{H}$ NMR spectra of the aromatic region of $1 \mathrm{a}$ at concentrations of $0.0285,0.285$, and $2.85 \mathrm{mM}$ in $\mathrm{CD}_{3} \mathrm{CN}$. The relatively small changes observed between the 2.85 and $0.285 \mathrm{mM}$ samples suggest that $\mathrm{PF}_{6}{ }^{-}$anions remain bound inside $\mathbf{1 a}$ at both concentrations. The shifting of specific resonances - marked by dotted lines - upon further dilution is attributed to the weakening of anion binding inside the cage at this lowest concentration $(0.0285 \mathrm{mM}$ of 1a). These results are consistent with the ${ }^{19} \mathrm{~F}$ NMR spectra from these experiments (Figure S17). Further confirming this interpretation, the ${ }^{1} \mathrm{H}$ NMR resonance of 1 a that shifts most upon dilution corresponds to the aromatic protons $\mathrm{F}$, which are closest to the $\mathrm{PF}_{6}{ }^{-}$anions inside of the cage in the solid state (see Figure S16). 
Cage 1a ${ }^{1} \mathrm{H}$ NMR, $500 \mathrm{MHz}, \mathrm{CD}_{3} \mathrm{CN}$

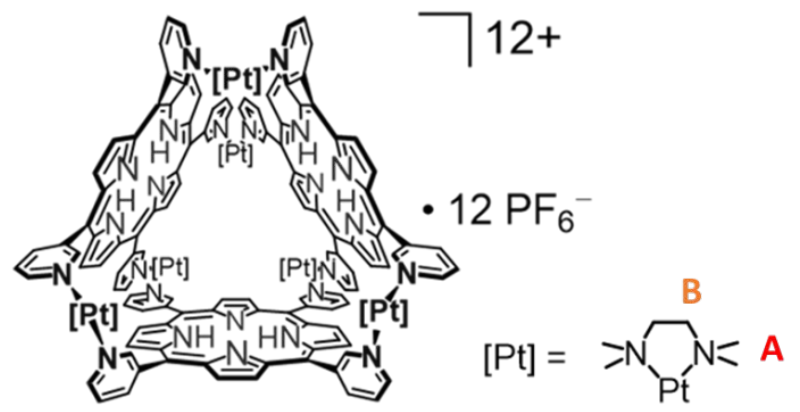

$1 a$

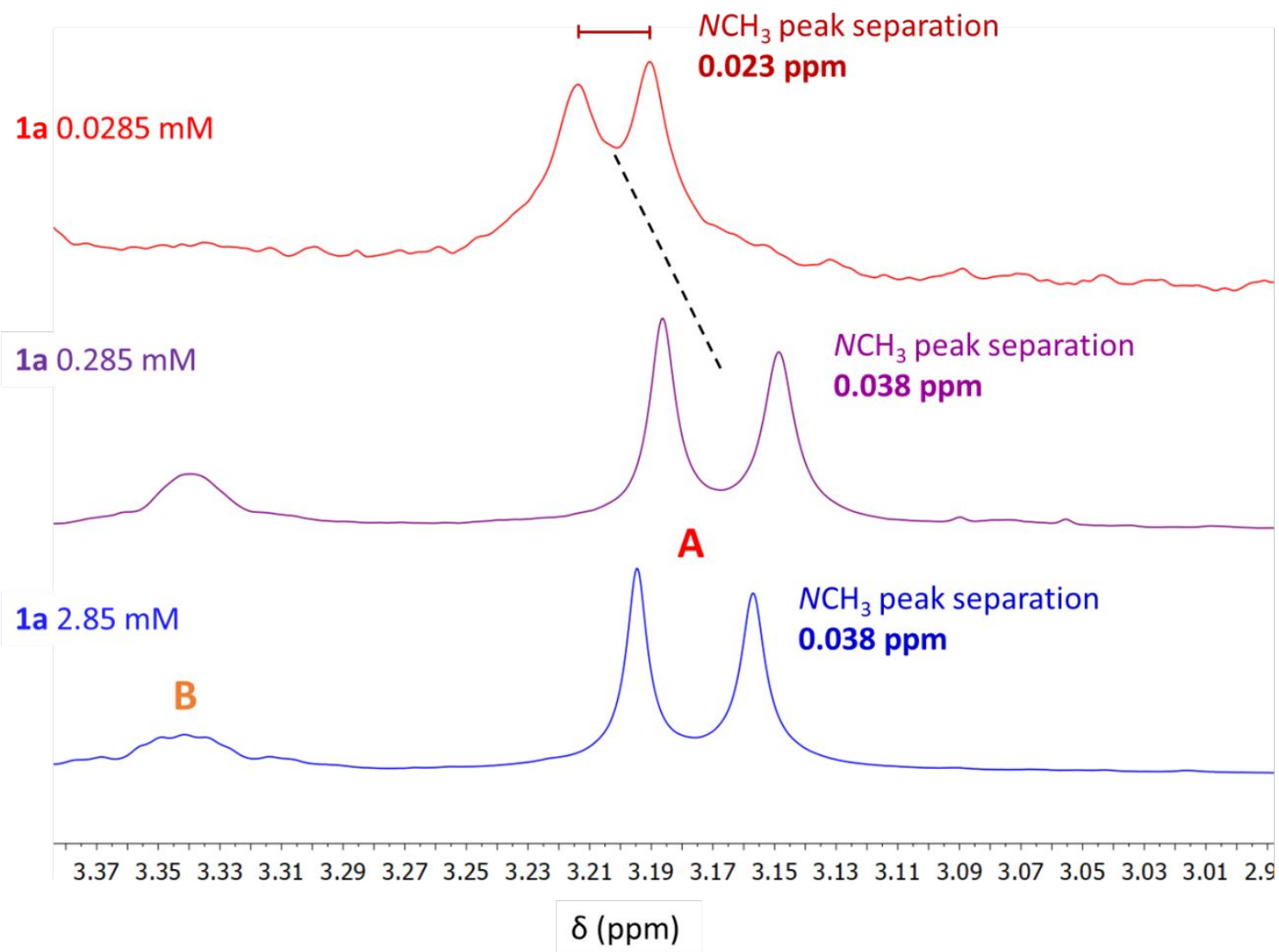

Figure S19: Stacked ${ }^{1} \mathrm{H}$ NMR spectra of the aliphatic region of $1 \mathrm{a}$ at concentrations of 0.0285 , 0.285 , and $2.85 \mathrm{mM}$ in $\mathrm{CD}_{3} \mathrm{CN}$. The relatively small changes in the TMEDA $\mathrm{NCH}_{3}$ resonances observed between the 2.85 and $0.285 \mathrm{mM}$ samples suggest that $\mathrm{PF}_{6}{ }^{-}$anions remain similarly associated with 1a at both concentrations. The shifting of the methyl resonances upon further dilution is attributed to diminished anion association with the cage at this lowest concentration (0.0285 $\mathrm{mM}$ of $\mathbf{1 a}$ ). Consistent with this interpretation, the solid-state superstructure of $\mathbf{1 a}$ indicates that $\mathrm{PF}_{6}{ }^{-}$anions preferentially associate near the $\mathrm{NCH}_{3}$ groups of the linkers (see Figure S16). 
Cage $1 b^{19} \mathrm{~F} N M R, 470 \mathrm{MHz}, \mathrm{CD}_{3} \mathrm{CN}$

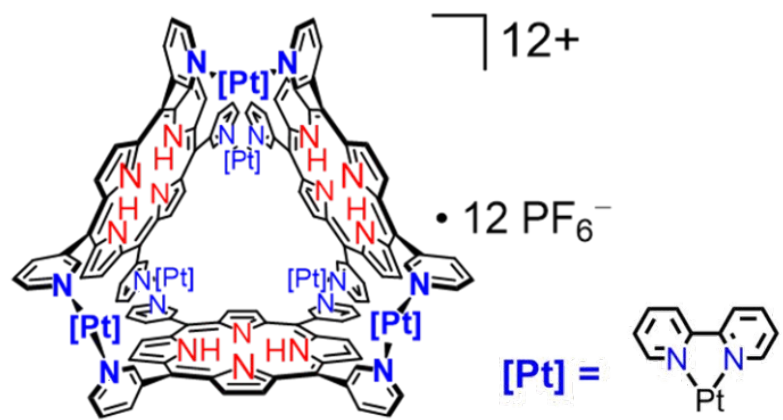

$1 \mathrm{~b}$

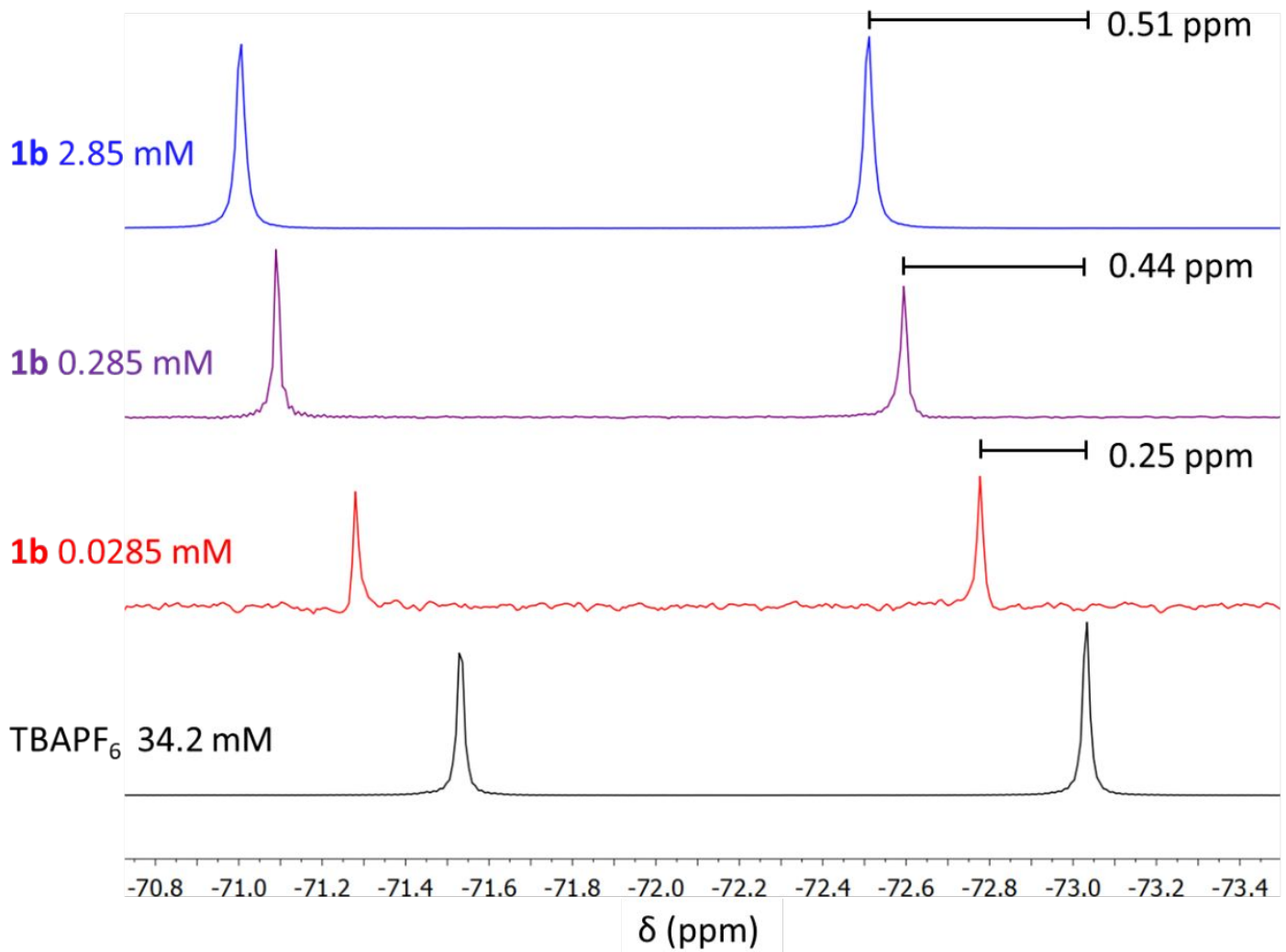

Figure S20: ${ }^{19} \mathrm{~F}$ NMR spectra $\left(\mathrm{CD}_{3} \mathrm{CN}\right)$ showing the shift of the $\mathrm{PF}_{6}{ }^{-}$resonance in a $2.85 \mathrm{mM}$ sample of $\mathbf{1 b}$ upon 10 -fold and 100 -fold dilution. The ${ }^{19} \mathrm{~F}$ NMR spectrum of a $34.2 \mathrm{mM}$ sample of TBAPF 6 in $\mathrm{CD}_{3} \mathrm{CN}$ is provided for comparison. The $\mathrm{PF}_{6}{ }^{-}$concentration in this latter sample matches that in the $2.85 \mathrm{mM}$ samples of $\mathbf{1 b}$, but the $\mathrm{TBAPF}_{6}$ spectrum depicts the chemical shift of the unbound $\mathrm{PF}_{6}{ }^{-}$anions since $\left[\mathrm{NBu}_{4}\right]^{+}$cations do not interact strongly with these anions. Thus, differences between the ${ }^{19} \mathrm{~F}$ NMR spectra of $\mathbf{1} \mathbf{b}$ and that of $\mathrm{TBAPF}_{6}$ are indicative of the association of $\mathrm{PF}_{6}{ }^{-}$ with $\mathbf{1 b}$. Dilution weakens the binding of the anions as evident from the ${ }^{19} \mathrm{~F}$ NMR resonances of $\mathrm{PF}_{6}{ }^{-}$shifting towards its unbound value upon dilution of a sample of $\mathbf{1} \mathbf{b}$. In control experiments, dilution of samples of $\mathrm{TBAPF}_{6}$ did not result in significant changes to the ${ }^{19} \mathrm{~F}$ NMR chemical shift of the $\mathrm{PF}_{6}{ }^{-}$resonance. 
Cage $1 \mathbf{b}^{1} \mathrm{H} N M R, 500 \mathrm{MHz}, \mathrm{CD}_{3} \mathrm{CN}$

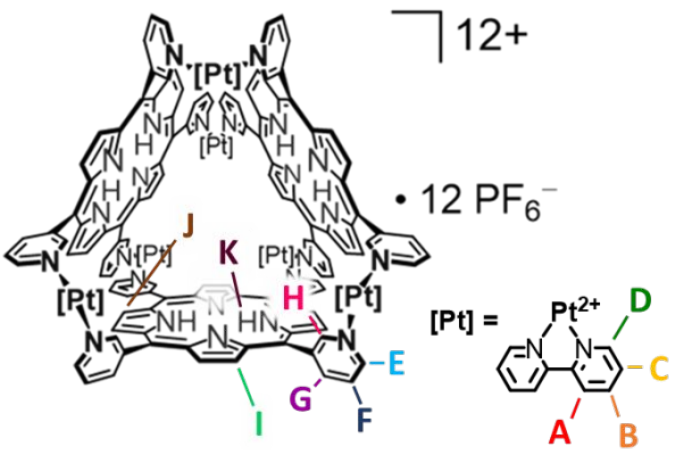

$1 \mathrm{~b}$

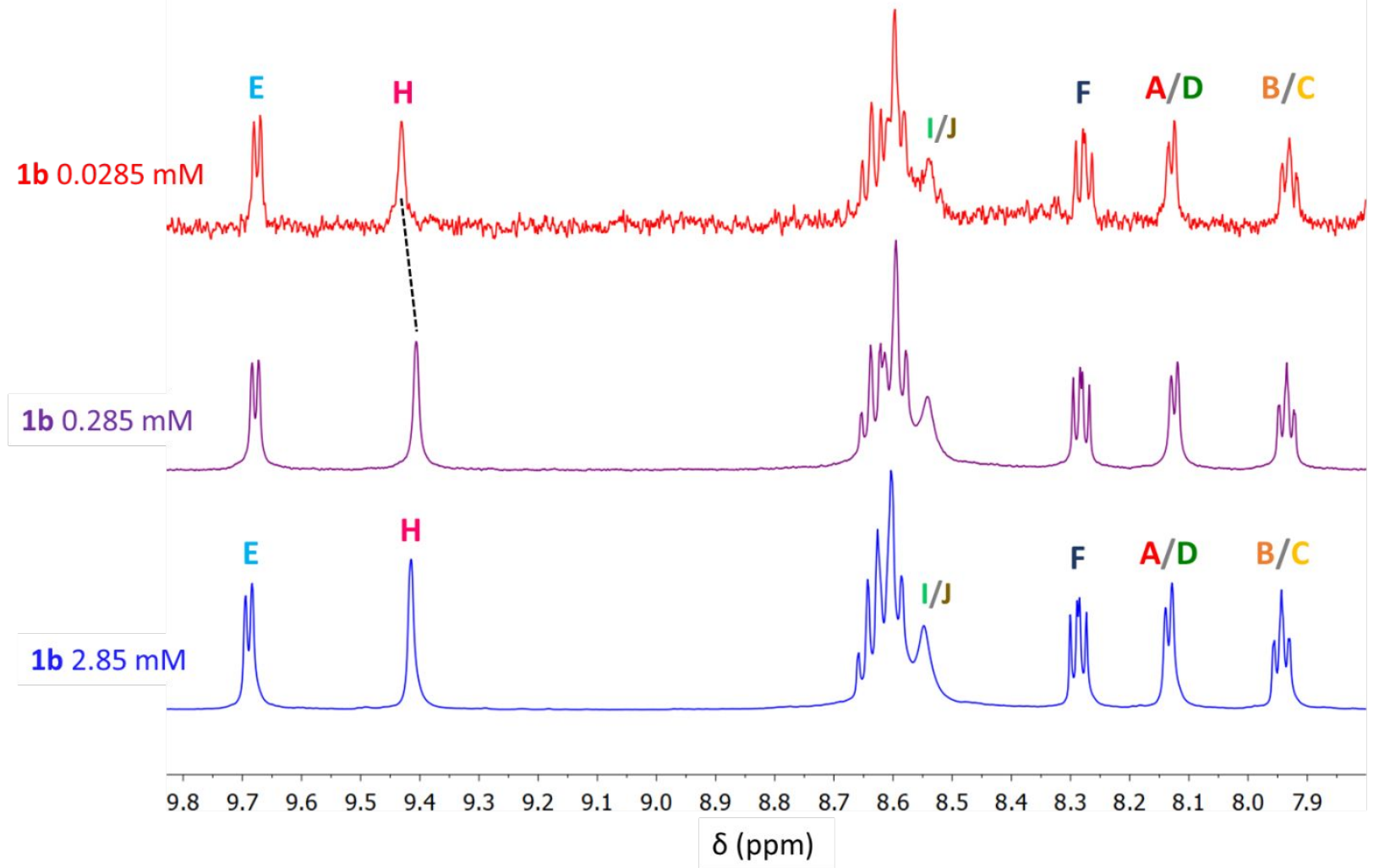

Figure S21: ${ }^{1} \mathrm{H}$ NMR spectra of the aromatic region of $\mathbf{1 b}$ at concentrations of $0.0285,0.285$, and $2.85 \mathrm{mM}$ in $\mathrm{CD}_{3} \mathrm{CN}$. The relatively small changes observed between the 2.85 and $0.285 \mathrm{mM}$ samples suggest that $\mathrm{PF}_{6}{ }^{-}$anions remain bound inside $\mathbf{1 b}$ at both concentrations. The shifting of proton $\mathbf{H}$ (indicated with a black dotted line) upon further dilution is attributed to diminished anion binding inside the cage at this lowest concentration $(0.0285 \mathrm{mM}$ of $\mathbf{1 b})$. These results are consistent with the ${ }^{19} \mathrm{~F}$ NMR spectra from these experiments (Figure S20). 
Cage $1 b^{1} \mathrm{H} N M R, 500 \mathrm{MHz}, \mathrm{CD}_{3} \mathrm{CN}$

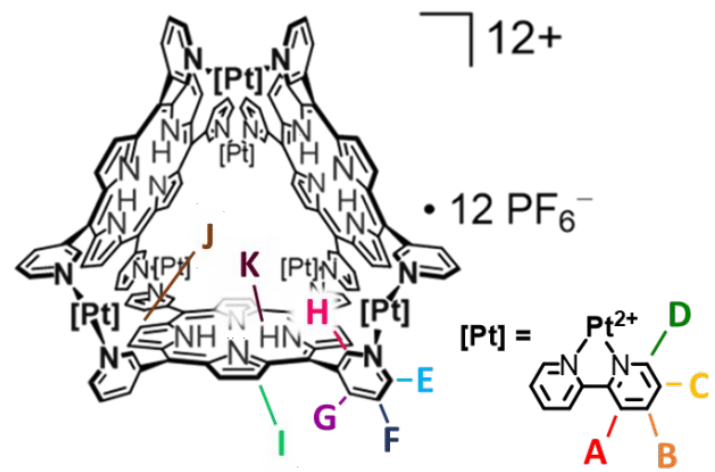

$1 b$

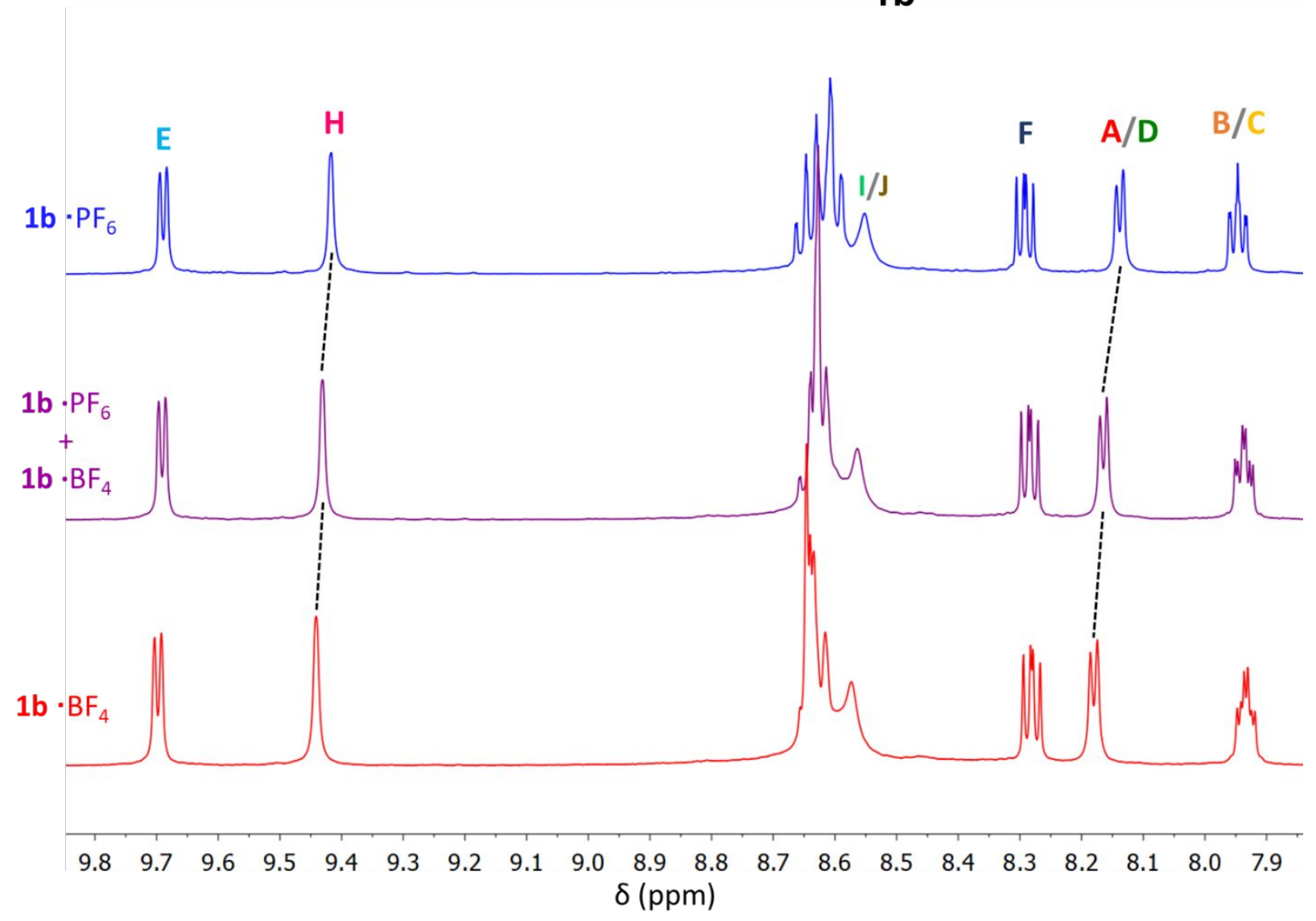

Figure S22: Stacked ${ }^{1} \mathrm{H}$ NMR spectra (in $\mathrm{CD}_{3} \mathrm{CN}$ ) of the aromatic region of $1.7 \mathrm{mM}$ solutions of $\mathbf{1 b}$ as its $\mathbf{P F}_{6}{ }^{-}$salt (blue), a 1:1 mixture of $\mathbf{1} \mathbf{b} \cdot \mathbf{P F}_{\mathbf{6}}$ and $\mathbf{1} \mathbf{b} \cdot \mathbf{B} \mathbf{F}_{\mathbf{4}}$ (purple), and $\mathbf{1} \mathbf{b} \cdot \mathbf{B F}_{\mathbf{4}}$ (red). The largest changes (indicated with black dotted lines) are observed for resonance $\mathrm{H}$, which corresponds to the 3-py proton facing the interior of the cage, and resonance A/D which is assigned to the bipy ligand of the linkers (note that this latter resonance could not be definitively assigned between two positions on the bipy ligand that give rise to similar coupling patterns). 
Cage $1 b^{1} \mathrm{H}$ NMR, $500 \mathrm{MHz}, \mathrm{CD}_{3} \mathrm{CN}$

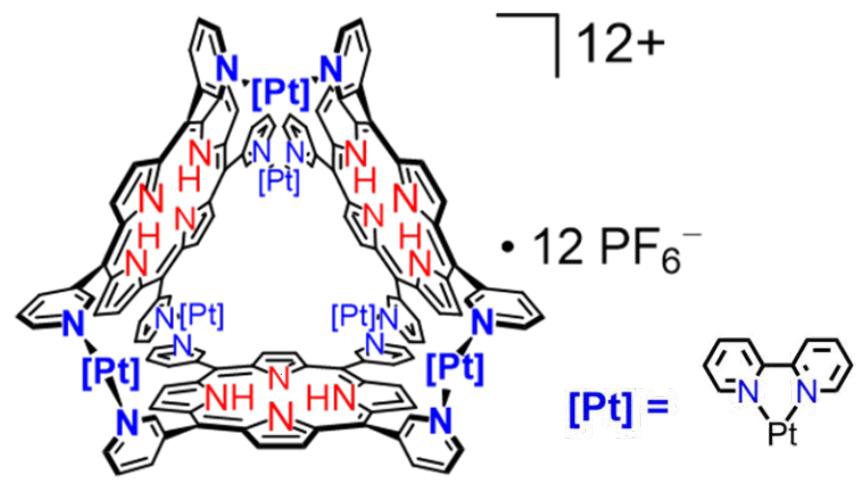

$0.578 \mathrm{mM} \mathrm{1b} \cdot \mathrm{PF}_{6}$
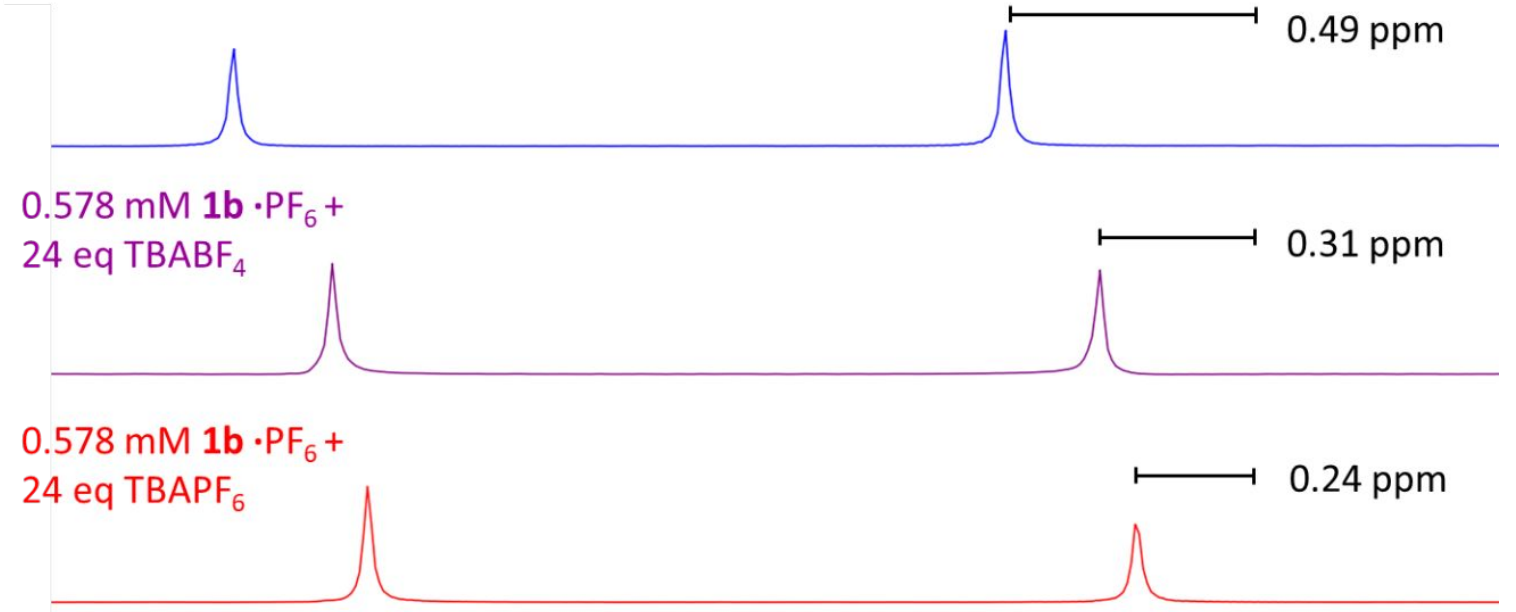

$34.2 \mathrm{mM}$

TBAPF $_{6}$

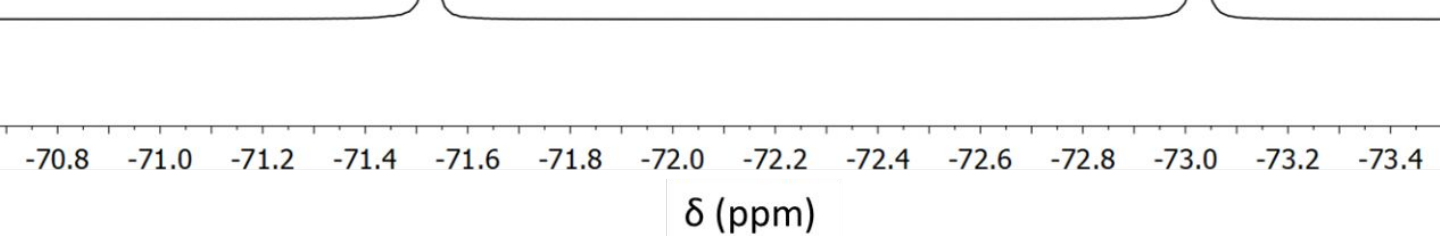

Figure S23: Stacked ${ }^{19} \mathrm{~F} N M R$ spectra $\left(\mathrm{CD}_{3} \mathrm{CN}\right)$ showing the shift of the $\mathrm{PF}_{6}{ }^{-}$resonance of 0.578 $\mathrm{mM} \mathbf{1 b} \cdot \mathbf{P F}_{6}$ (blue), $0.578 \mathrm{mM} \mathbf{1 b} \cdot \mathbf{P F}_{6}$ with 24 equivalents of TBABF 4 added (purple), $0.578 \mathrm{mM}$ 1b. PF $_{6}$ with 24 equivalents of $\operatorname{TBAPF}_{6}$ (red), and $34.2 \mathrm{mM} \mathrm{TBAPF}_{6}$ (black). The $\mathrm{PF}_{6}{ }^{-}$resonances shifted towards the unbound value of $\mathrm{PF}_{6}{ }^{-}$upon the addition of both $\mathrm{TBABF}_{4}$ and $\mathrm{TBAPF}_{6}$, suggesting fast anion exchange in and out of the cage in both cases. The greater shift upon addition of $\mathrm{TBAPF}_{6}$ vs. $\mathrm{TBABF}_{4}$ suggests that $\mathrm{PF}_{6}{ }^{-}$binds more strongly than $\mathrm{BF}_{4}{ }^{-}$inside $\mathbf{1 b}$. 


$$
\begin{gathered}
k_{1 \boldsymbol{a}}=\frac{1}{\tau_{\text {coalescense }}}=\sqrt{2} * \pi * \Delta v_{P F_{6}} \\
k_{1 \boldsymbol{a}}=\sqrt{2} * \pi *(4.08 \mathrm{ppm} * 470 \mathrm{MHz}) \\
k_{\mathbf{1} \boldsymbol{a}}=8520 \mathrm{~s}^{-1}
\end{gathered}
$$

Equation S1: Sample calculation for determining the minimum rate of exchange between $\mathrm{PF}_{6}{ }^{-}$ anions in bulk solution and those bound inside 1a necessary to observe coalescence of the two signals. ${ }^{16}$ Since narrow ${ }^{19} \mathrm{~F}$ NMR line widths were observed for the $\mathrm{PF}_{6}{ }^{-}$anions in all experiments, the true rate of exchange must be much higher than this minimum estimate, indicating that anion exchange is not a rate limiting step during CV experiments at all scan rates employed. Likewise, a minimum $k_{1 \boldsymbol{b}}$ of $5763 \mathrm{~s}^{-1}$ was determined for anion movement in and out of $\mathbf{1 b}$.

\section{Cyclic Voltammetry}

Electrochemical experiments were performed with a $\mathrm{CH}$ Instruments 600E potentiostat. Dry MeCN was used as the solvent, and $0.1 \mathrm{M}$ tetrabutylammonium hexafluorophosphate (TBAPF 6 ) was used as a supporting electrolyte in all cases. Cyclic voltammetry was conducted using a $3 \mathrm{~mm}$ glassy carbon working electrode, a platinum wire counter electrode, and a silver wire pseudoreference electrode which was confined in a polypropylene body that provided contact with the analyte solution via a porous zeolite bead or a glass frit. Potentials were referenced to the $\mathrm{Fc}^{+/ 0}$ couple of an internal ferrocene standard or by calibrating the pseudo-reference electrode vs. the $\mathrm{Fc}^{+/ 0}$ couple of an external solution of ferrocene immediately before use (note: keeping the pseudo-reference electrode isolated from the bulk sample prevents significant drift of the reference potential over numerous CV experiments). For clarity, we present the CVs that do not include the $\mathrm{Fc}^{+/ 0}$ wave, and in all cases the measured potentials of redox processes were consistent with those determined with the internal $\mathrm{Fc}^{+/ 0}$ redox couple included along with the analyte. Positive feedback iR compensation was applied during all CV experiments. The $2^{\text {nd }}$ cycle out of three is presented unless otherwise noted. 


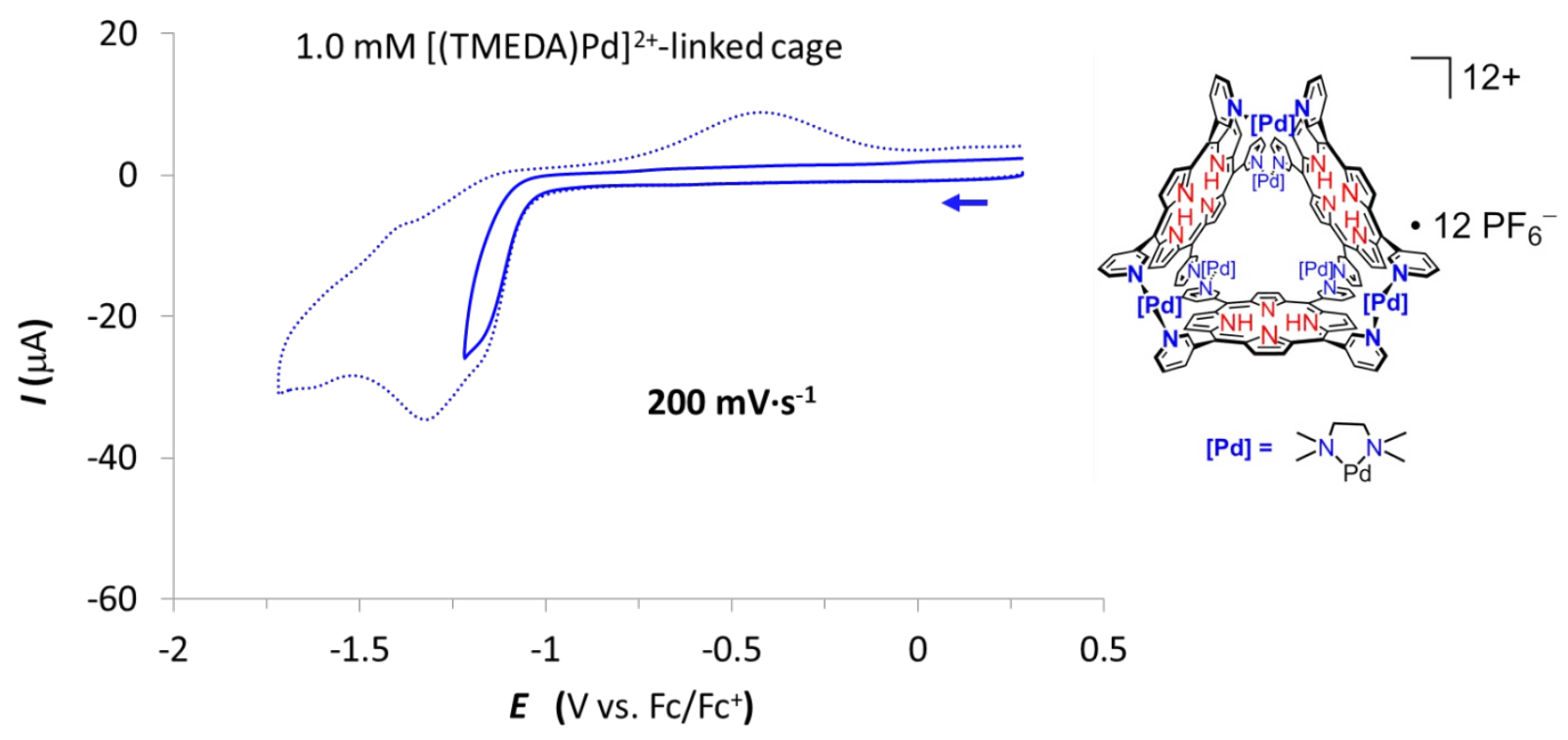

Figure S24: Cyclic voltammograms ( $1^{\text {st }}$ cycles shown) of the palladium-analogue of $1 \mathrm{a}$ at a scan rate of $200 \mathrm{mV} \cdot \mathrm{s}^{-1}$ and potential windows of -0.25 to $-1.22 \mathrm{~V}$ and -0.25 to $-1.72 \mathrm{~V}\left(\mathrm{vs}^{\mathrm{F}} \mathrm{Fc}^{+/ 0}\right.$ ). Reduction waves are irreversible within both of the potential windows examined.

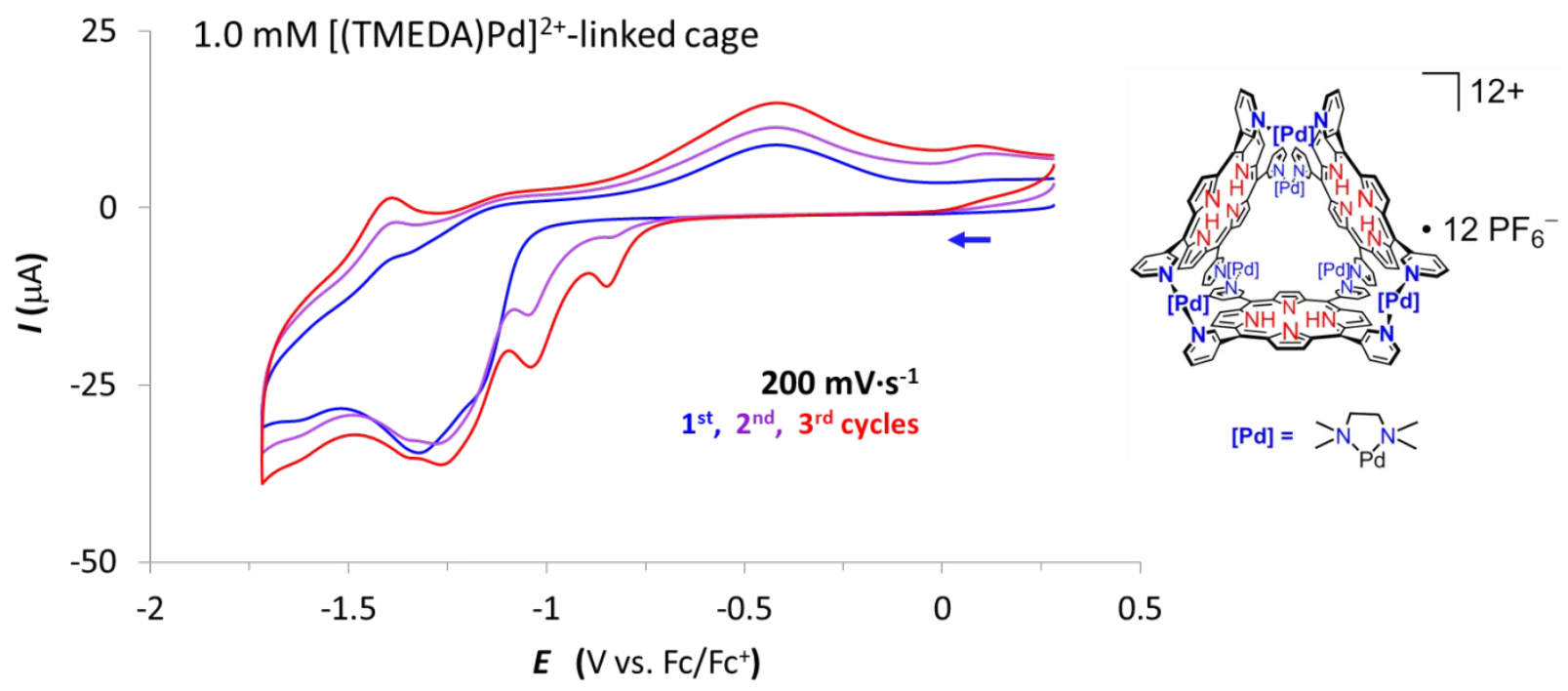

Figure S25: Cyclic voltammograms of the palladium-analogue of 1a showing three consecutive cycles. Inconsistent cycle-to-cycle behavior, including the appearance of new reduction waves on the second and third cycles, is attributed to the degradation of the cage, consistent with the cyclic voltammograms reported for other $[(N N) P d]^{2+}$ linked cages. ${ }^{17}$ 


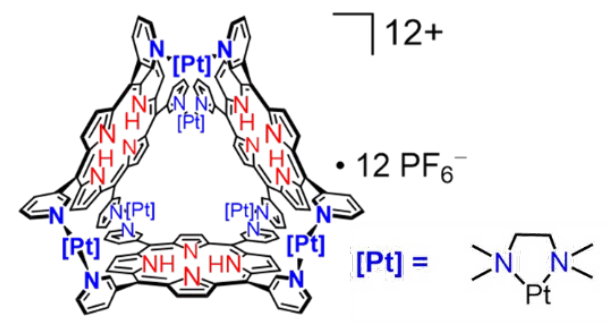

$1 \mathrm{a}$
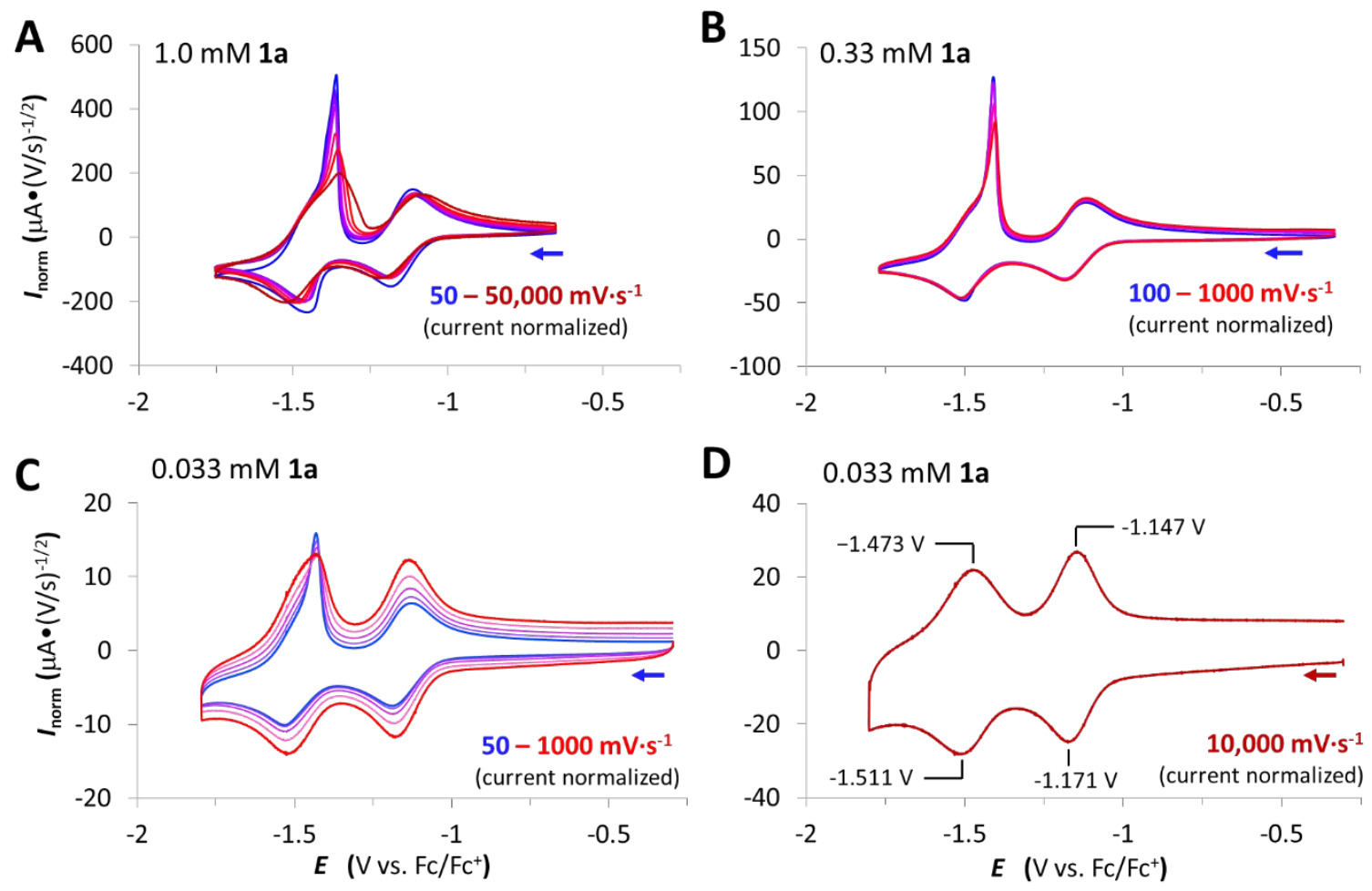

Figure S26: Cyclic voltammograms ( $2^{\text {nd }}$ cycle unless otherwise noted) of cage 1a: (A) $1.0 \mathrm{mM}$ analyte concentration measured at scan rates of $0.050,0.10,0.20,0.50,1.0,5.0,10.0$, and 50.0 $\mathrm{V} \cdot \mathrm{s}^{-1}$; (B) $0.33 \mathrm{mM}$ analyte concentration measured at scan rates of $0.10,0.20,0.50$, and $1.0 \mathrm{~V} \cdot \mathrm{s}^{-}$ 1; (C) $0.033 \mathrm{mM}$ analyte concentration measured at scan rates of $0.050,0.10,0.20,0.50$, and 1.0 $\mathrm{V} \cdot \mathrm{s}^{-1}$; and (D) First cycle of a $0.033 \mathrm{mM}$ analyte sample recorded at a scan rate of $10.0 \mathrm{~V} \cdot \mathrm{s}^{-1}$, showing the narrowest $\Delta E_{\mathrm{p}}$ values measured for $1 \mathrm{a}$. The sharp oxidative feature observed near $-1.4 \mathrm{~V}$ in $\mathrm{A}-\mathrm{C}$ is attributed to the reoxidation of surface-adsorbed $1 \mathrm{a}$. The decrease in the current of this feature at high scan rates is attributed to the faster timescale of the experiments, which leaves less time for the reduced cage to adsorb on the electrode prior to reoxidation. Likewise, lowering the concentration of $\mathbf{1 a}$ to $0.033 \mathrm{mM}$ appears to inhibit surface adsorption such that solely diffusion-controlled redox waves are observed at scan rates of $\geq 1 \mathrm{~V} \cdot \mathrm{s}^{-1}$. 


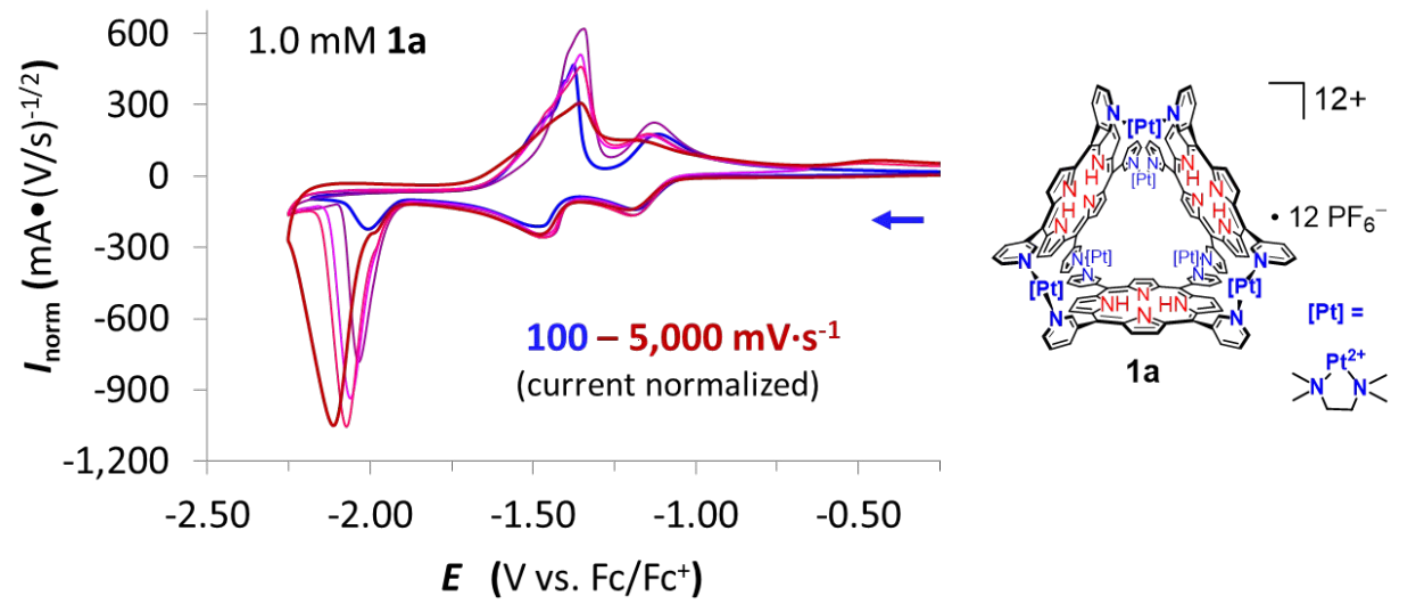

Figure S27: Cyclic voltammograms of a $1.0 \mathrm{mM}$ solution of 1a measured at scan rates of 0.050 , $0.10,0.20,0.50,1.0$, and $5.0 \mathrm{~V} \cdot \mathrm{s}^{-1}$ showing a potential window extending to $-2.25 \mathrm{~V}\left(\mathrm{vs}^{\mathrm{F}} \mathrm{Fc}^{+/ 0}\right)$. A sharp, irreversible reduction wave appears negative of $-2.0 \mathrm{~V}$ and is attributed to linker-centered reductions of 1a that is adsorbed on the electrode surface. The current of this wave does not scale with the scan rate as expected for surface-adsorbed redox features, presumably because faster scan rates result in less material depositing on the electrode.

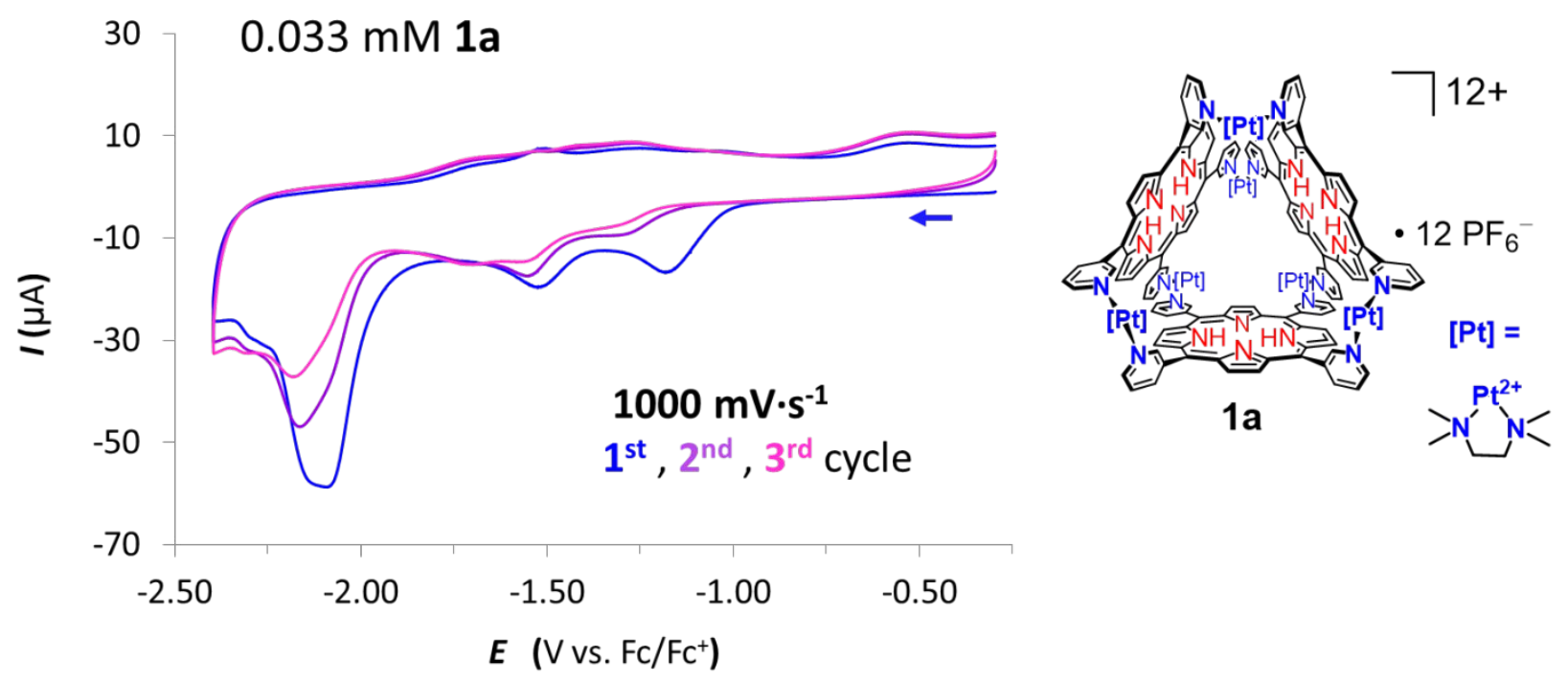

Figure S28: Cyclic voltammogram of a $0.033 \mathrm{mM}$ solution of 1a measured at a scan rate of $1 \mathrm{~V} \cdot \mathrm{s}^{-1}$ with a potential window that includes a large reduction wave at $<-2.0 \mathrm{~V}\left(\mathrm{vs}\right.$. $\left.\mathrm{Fc}^{+/ 0}\right)$. Three consecutive cycles are shown, revealing that this third reduction wave eliminates the reversibility of the first two reductions and causes a significant decrease of current for each subsequent cycle. These changes suggest that the third reduction of 1a induces decomposition of the cage. 


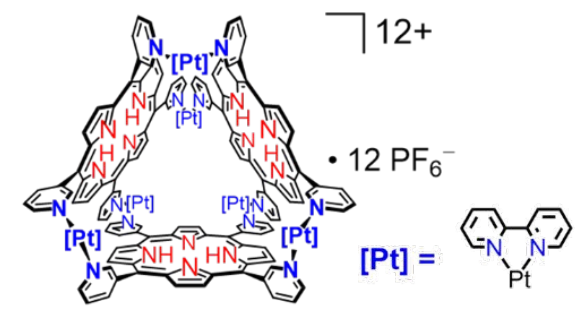

1b
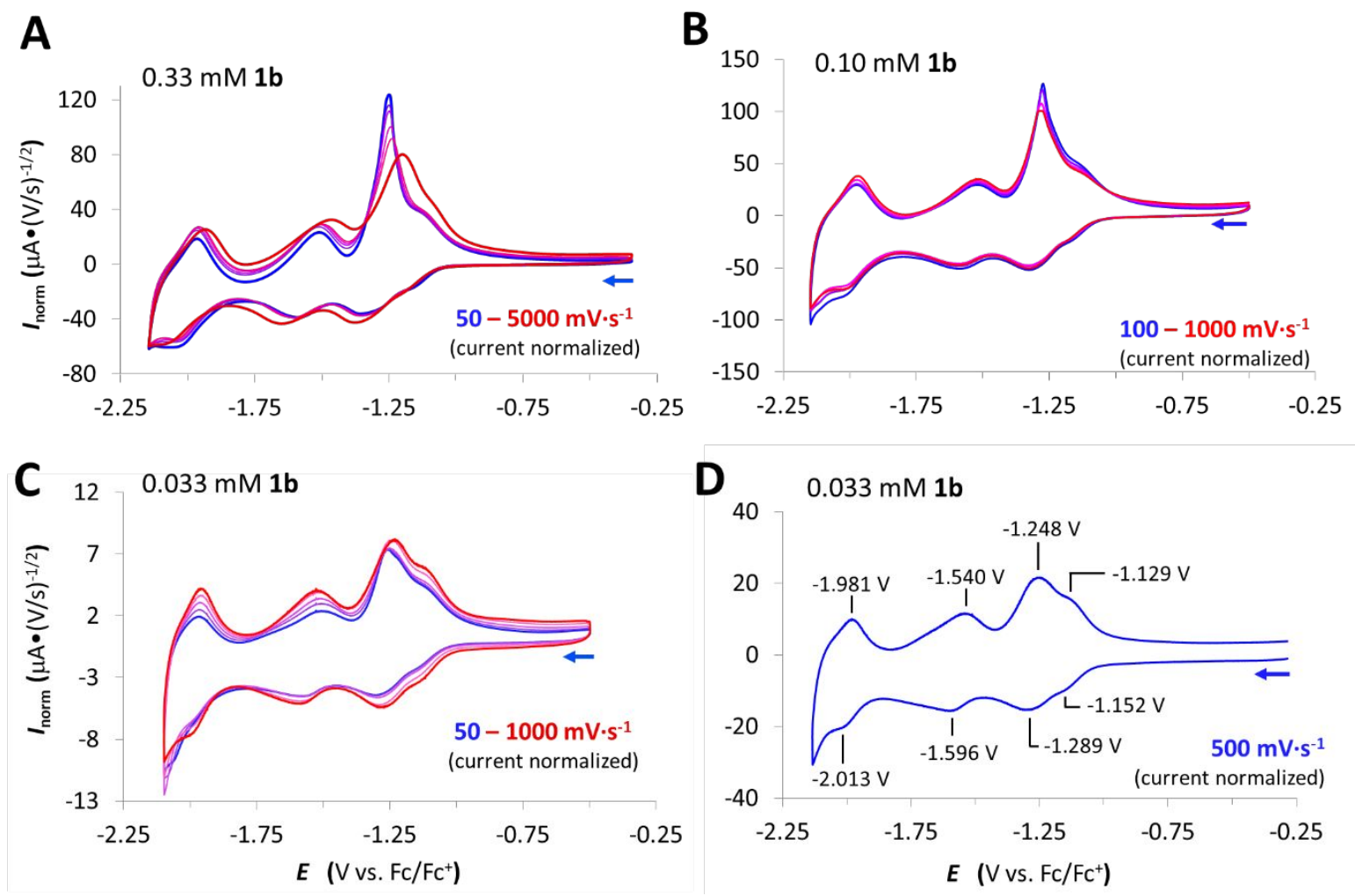

Figure S29: Cyclic voltammograms ( $2^{\text {nd }}$ cycles unless otherwise noted) of $(A) 0.33 \mathrm{mM} \mathbf{~ b ~ b}$ measured at scan rates of $0.050,0.10,0.20,0.50,1.0$, and $5.0 \mathrm{~V} \cdot \mathrm{s}^{-1}$; (B) $0.1 \mathrm{mM} 1 \mathrm{~b}$ measured at

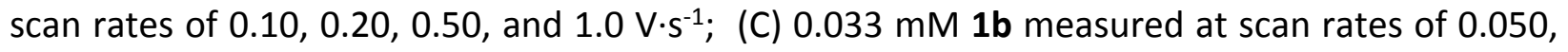
$0.10,0.20,0.50,1.0 \mathrm{~V} / \mathrm{s}$; and (D) First cycle of $0.033 \mathrm{mM}$ sample of $1 \mathbf{b}$ recorded at $0.50 \mathrm{~V} \cdot \mathrm{s}^{-1}$, showing the lowest $\Delta E_{\mathrm{p}}$ values measured for $1 \mathrm{~b}$. In $\mathrm{A} \& \mathrm{~B}$, the sharp oxidative features near -1.25 $V$ is attributed to the reoxidation of $\mathbf{1 b}$ that is adsorbed onto the electrode surface after reduction. The decrease in the current of this feature at high scan rates is attributed to the faster timescale of the experiments, which leaves less time for the reduced cage to adsorb on the electrode prior to reoxidation. Likewise, lowering the concentration of $1 \mathbf{b}$ to $0.033 \mathrm{mM}$ (C \& D) appears to inhibit surface adsorption such that the sharp reoxidation feature is eliminated entirely at scan rates $\geq 0.50 \mathrm{~V} \cdot \mathrm{s}^{-1}$, leaving only diffusion controlled redox waves. 


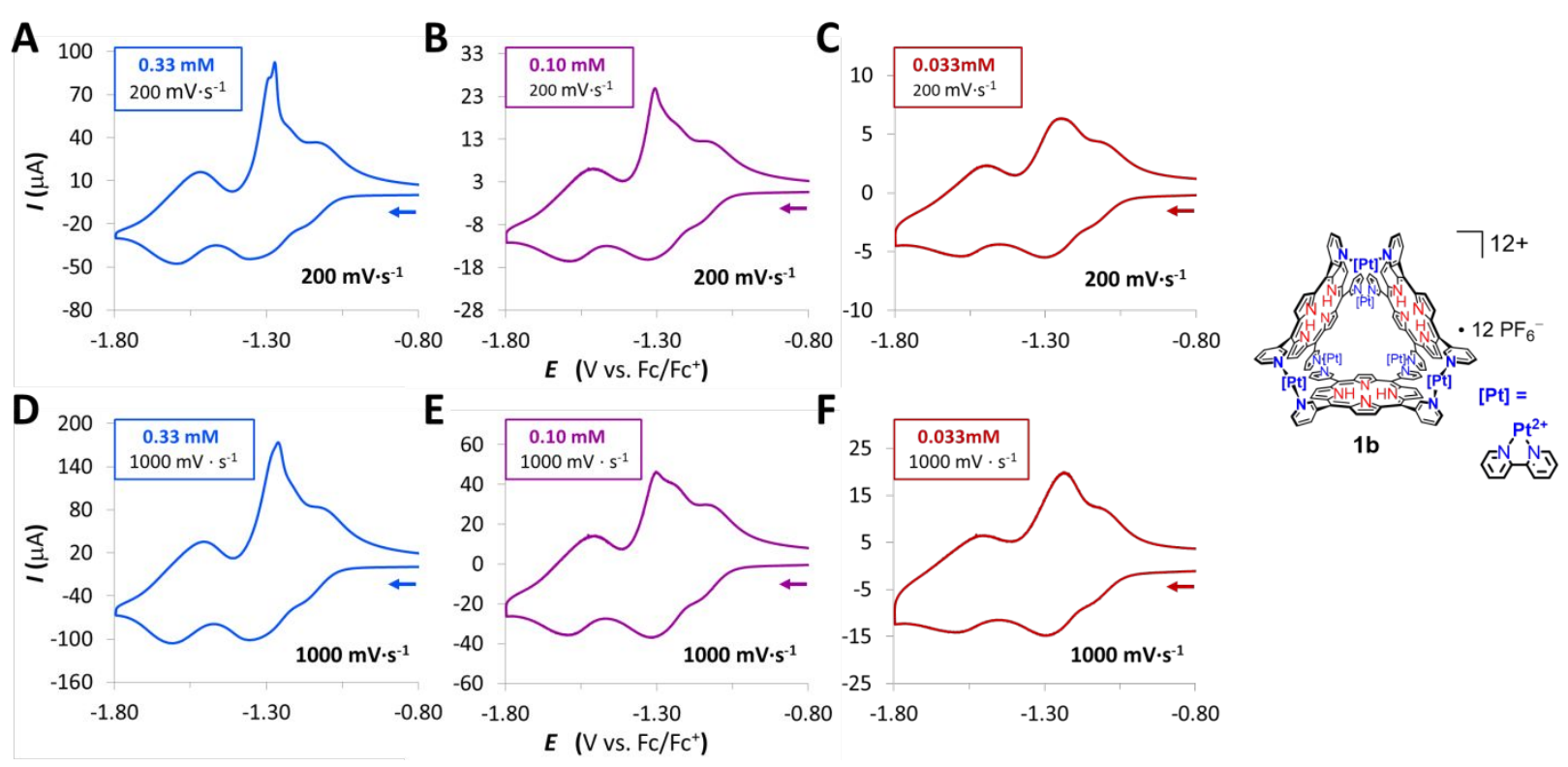

Figure S30: Cyclic voltammograms of $\mathbf{1 b}$ showing the concentration and scan rate dependence of the sharp reoxidation feature observed near $-1.25 \mathrm{~V}$ : (A) $0.33 \mathrm{mM} \mathrm{1b}, 0.20 \mathrm{~V} \cdot \mathrm{s}^{-1}$; (B) $0.10 \mathrm{mM}$ 1b, $0.20 \mathrm{~V} \cdot \mathrm{s}^{-1}$; (C) $0.033 \mathrm{mM} \mathrm{1b}, 0.20 \mathrm{~V} \cdot \mathrm{s}^{-1}$; (D) $0.33 \mathrm{mM} \mathrm{1b}, 1.0 \mathrm{~V} \cdot \mathrm{s}^{-1}$; (E) $0.10 \mathrm{mM} \mathrm{1b}, 1.0 \mathrm{~V} \cdot \mathrm{s}^{-1}$; (F) $0.033 \mathrm{mM} \mathrm{1b}, 1.0 \mathrm{~V} \cdot \mathrm{s}^{-1}$.

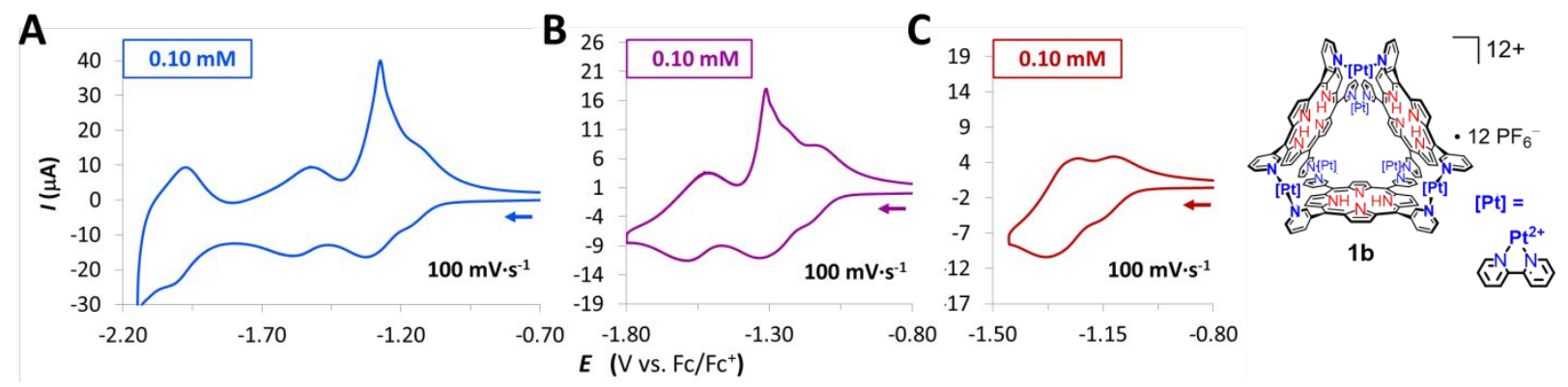

Figure S31: Cyclic voltammograms of a $0.10 \mathrm{mM}$ solution of $\mathbf{1 b}$ measured at a scan rate of 100 $\mathrm{mV} \cdot \mathrm{s}^{-1}$, showing potential windows extending to a minimum value of $(\mathrm{A})-2.10 \mathrm{~V},(\mathrm{~B})-1.80 \mathrm{~V}$, and (C) $-1.45 \mathrm{~V}$ (all vs. $\mathrm{Fc}^{+/ 0}$ ). The decrease in the current of the sharp reoxidation feature near -1.25 $\mathrm{V}$ is attributed to the shorter timescale of the CV experiments when the potential window is decreased, which provides less time for the analyte to adsorb on the electrode surface. 


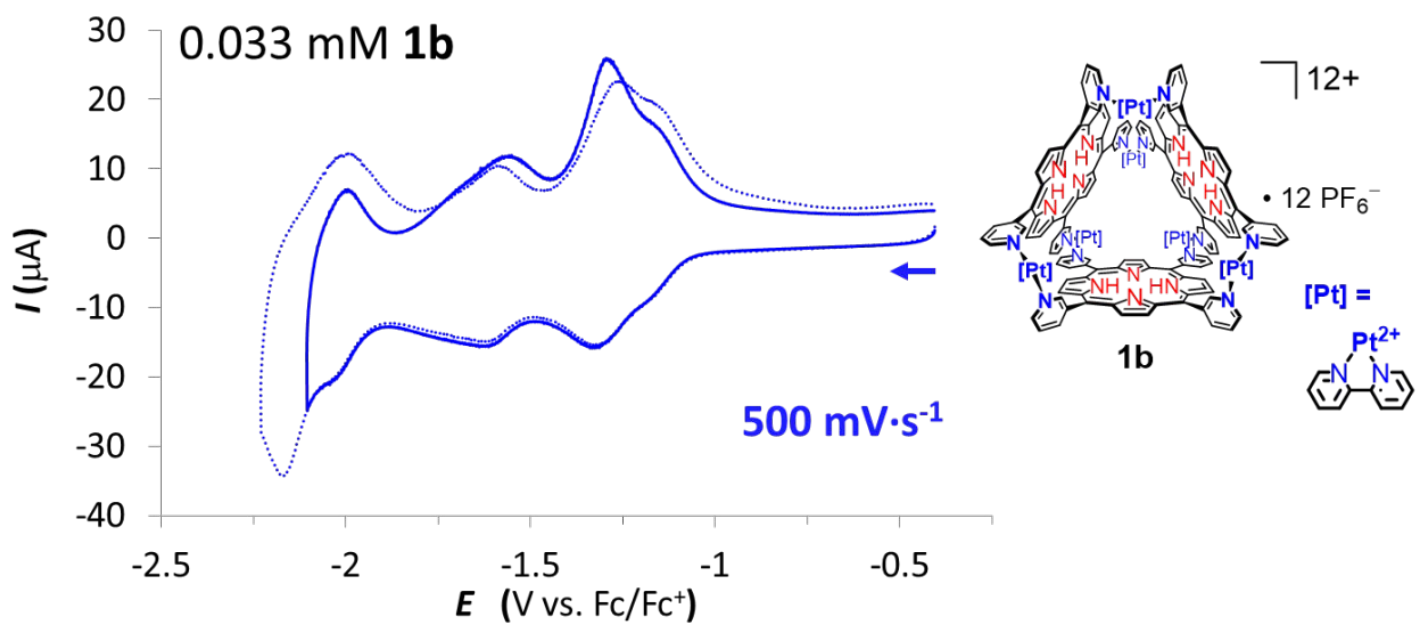

Figure S32: Cyclic voltammograms of a $0.033 \mathrm{mM}$ solution of $\mathbf{1 b}$ comparing potential windows with minimum values of -2.10 vs. $-2.20 \mathrm{~V}$ vs. $\mathrm{Fc}^{+/ 0}$. A fifth, quasireversible reduction is observed with the wider potential window. The corresponding reoxidation wave overlaps with that of the $4^{\text {th }}$ reversible reduction and causes only slight changes to the other reversible redox couples of 1b. Note that the two potential windows in this figure were measured on the same sample, providing even better agreement between the two potential windows than is presented in Figure 3 of the main text, which compares these to potential windows on different samples.

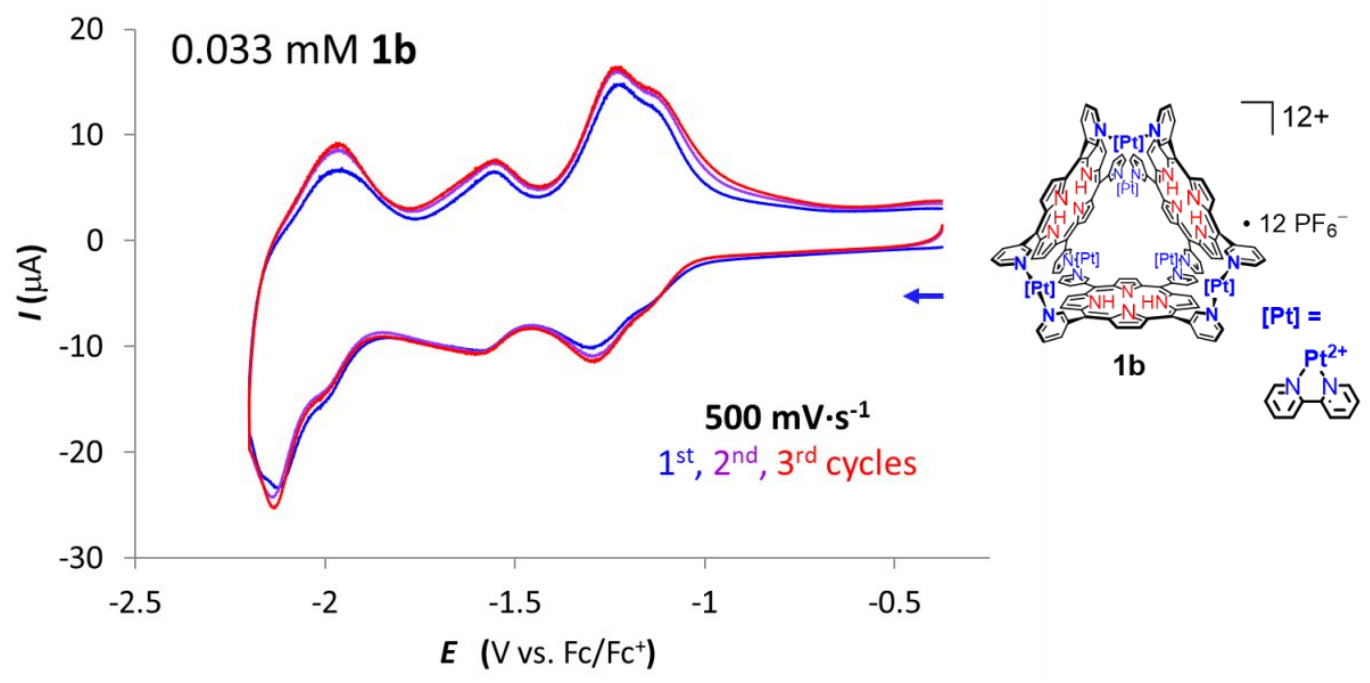

Figure S33: Cyclic voltammogram of a $0.033 \mathrm{mM}$ solution of $\mathbf{1 b}$ showing multiple cycles with the potential window extending from $-0.375 \mathrm{~V}$ to $-2.20 \mathrm{~V}$. The consistency of the $\mathrm{CV}$ from the $1^{\text {st }}$ to $3^{\text {rd }}$ cycles indicates that the cage structure remains stable through to the fifth reduction. 


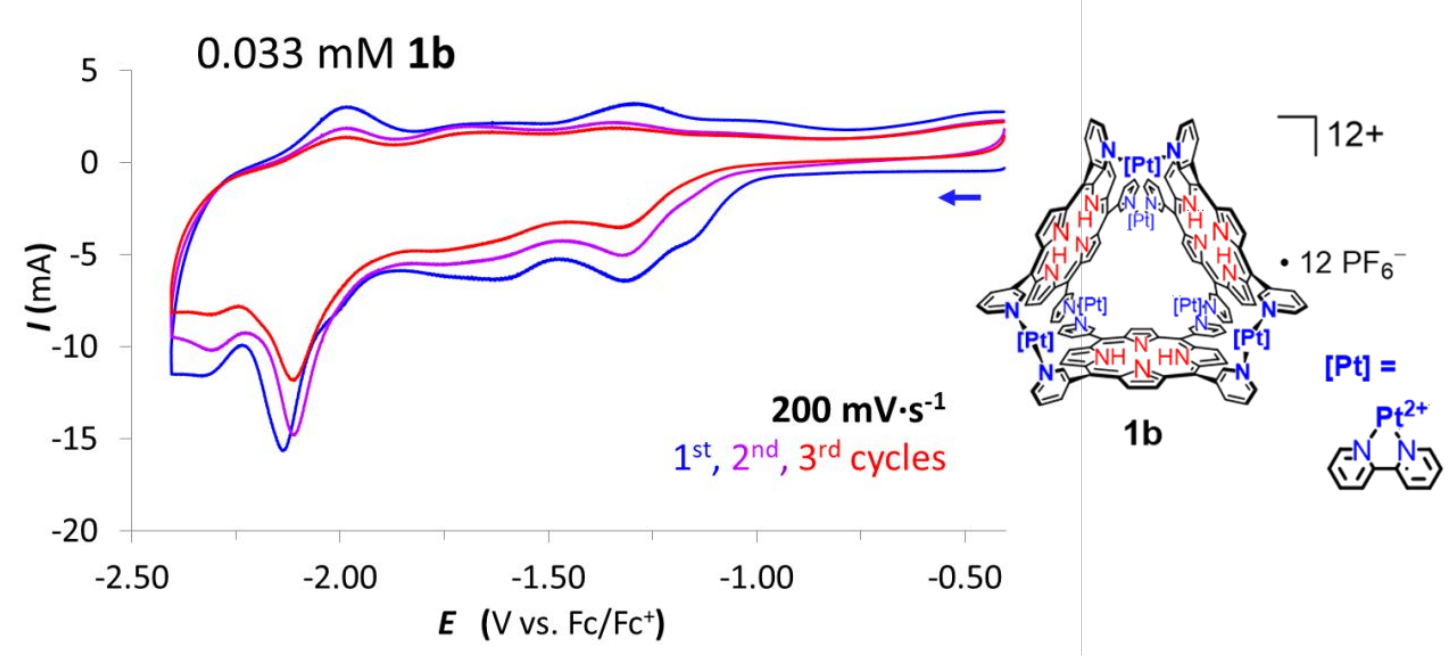

Figure S34: Cyclic voltammogram of a $0.033 \mathrm{mM}$ solution of $\mathbf{1 b}$ showing multiple cycles with the potential window extending from $-0.4 \mathrm{~V}$ to $-2.40 \mathrm{~V}$ vs $\mathrm{Fc}^{+/ 0}$. A sixth reduction occurs negative of -2.25 $\mathrm{V}$ eliminates the reversibility and cycle-to-cycle consistency of the other features, suggesting degradation of $\mathbf{1 b}$ and/or fouling of the electrode surface.

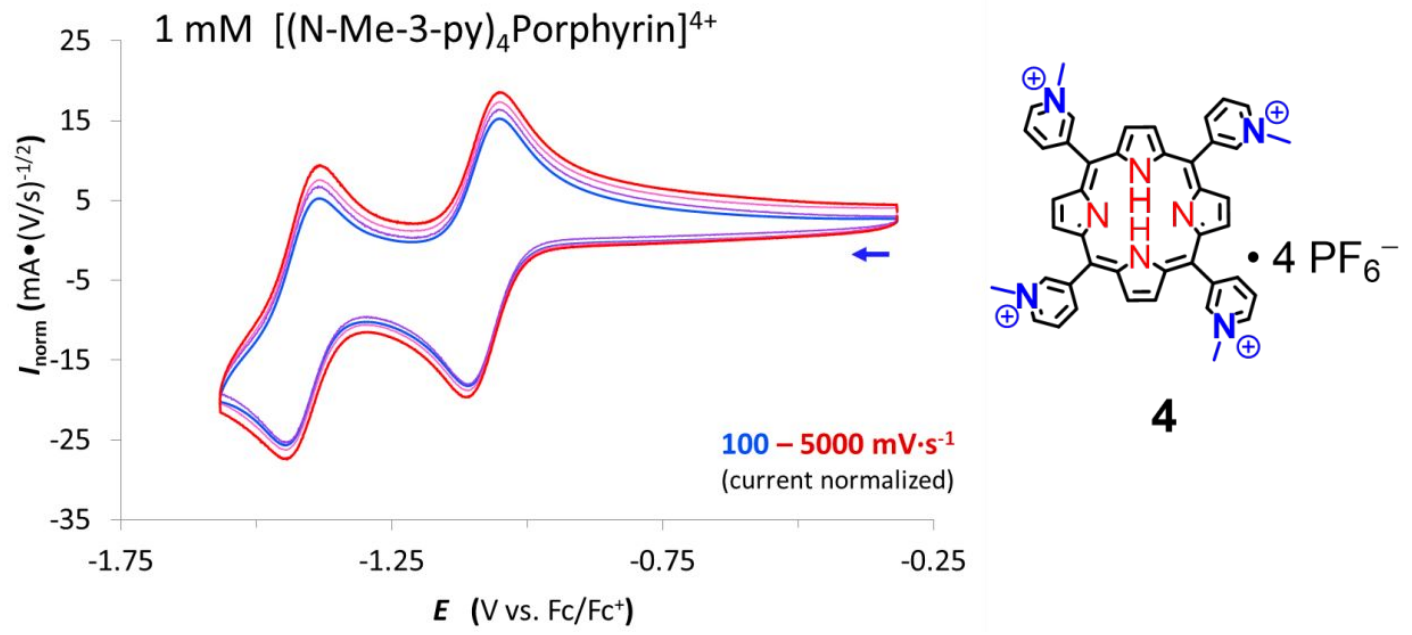

Figure S35: Cyclic voltammograms of a $1.0 \mathrm{mM}$ solution of [tetrakis(N-Me-3-pyridinium)porphyrin] $4 \mathrm{PF}_{6}(4)$ measured at scan rates of $0.10,0.50,1.0$, and $5.0 \mathrm{~V} \cdot \mathrm{s}^{-1}$. 


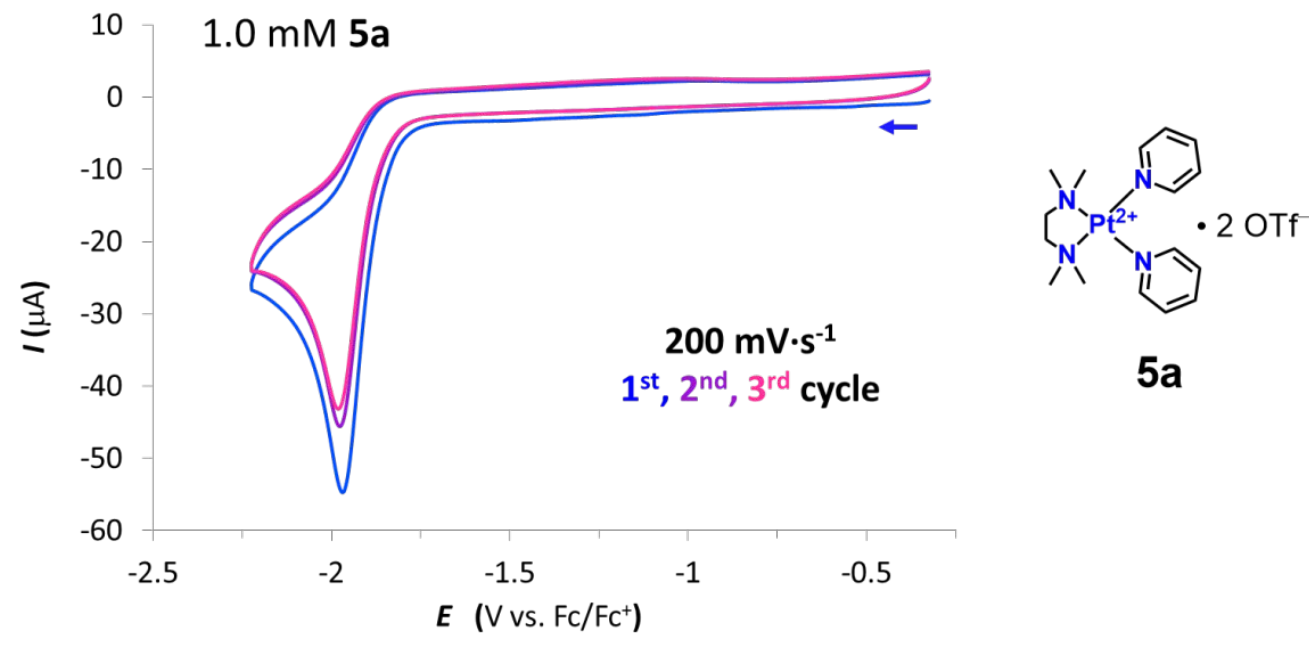

Figure S36: Cyclic voltammogram of a $1.0 \mathrm{mM}$ solution of 5a showing a decrease in current after the first cycle, confirming that the irreversibility of its reduction results from decomposition.

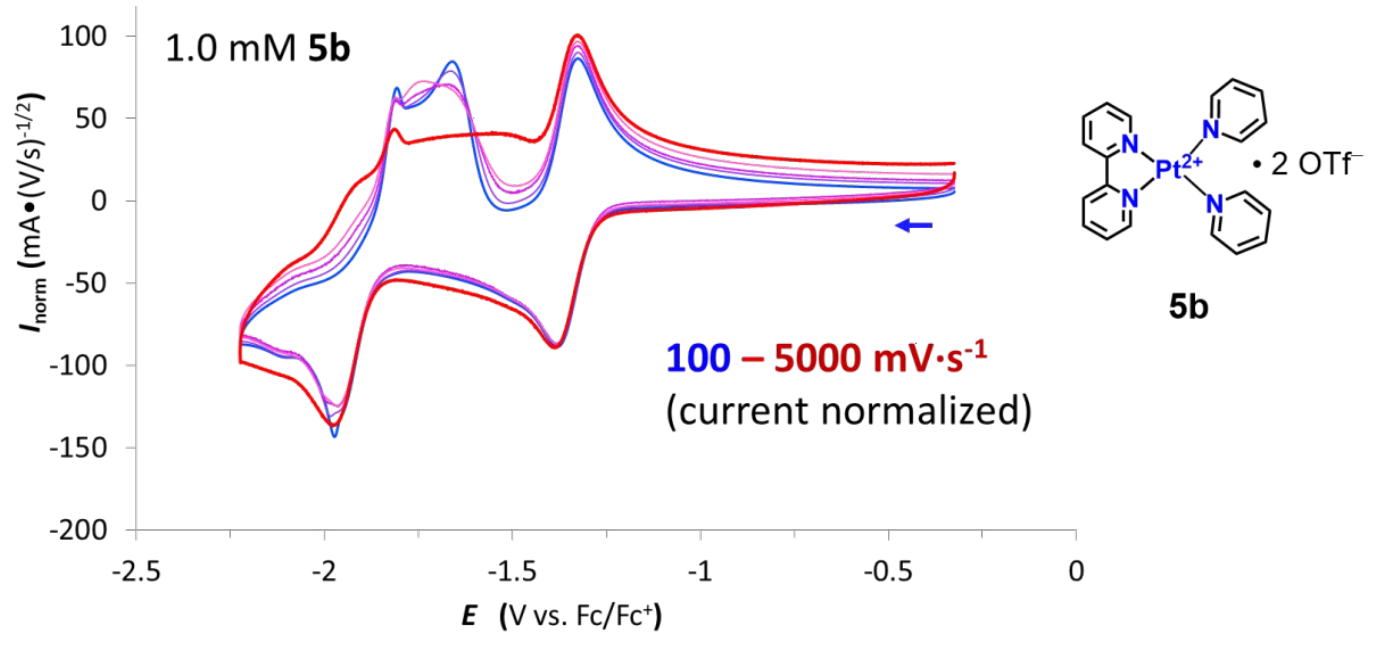

Figure S37: Cyclic voltammograms of a $1.0 \mathrm{mM}$ solution of $\mathbf{5 b}$ measured at scan rates of 0.10 , $0.20,0.50,1.0$, and $5.0 \mathrm{~V} \cdot \mathrm{s}^{-1}$. 


\section{Spectroelectrochemistry}

Spectroelectrochemistry (SEC) experiments employed an SEC-C Thin Layer Quartz spectroelectrochemical cell from ALS Co. Ltd. This cell has a $1 \mathrm{~mm}$ path length and was equipped with a platinum mesh working electrode. A platinum wire counter electrode and a silver wire pseudoreference electrode were housed in individual PTFE bodies equipped with porous glass frits. Dry $\mathrm{MeCN}$ was employed as a solvent with $0.1 \mathrm{M}$ tetrabutylammonium hexafluorophosphate $\left(\mathrm{TBAPF}_{6}\right)$ as the supporting electrolyte. A CV experiment was performed on samples in this cell to confirm the external calibration of the pseudo-reference electrode prior to each SEC experiments. UV-vis-NIR spectra were recorded using a Shimadzu UV-2600i spectrophotometer equipped with an ISR-2600Plus integrating sphere attachment with a $220-1400 \mathrm{~nm}$ wavelength range for absorbance measurements. For each experiment, an initial UV-Vis spectrum was obtained, then the sample was electrochemically reduced to the desired state by applying the appropriate potential for $3-5$ minutes, and UV-vis-NIR spectra were collected every 1 minute to monitor the reduction. The sample was reoxidized by applying a potential of $\geq+0.8 \mathrm{~V} v$ s. the most positive redox couple of the samples for 3-5 minutes while continuing to monitor the sample.

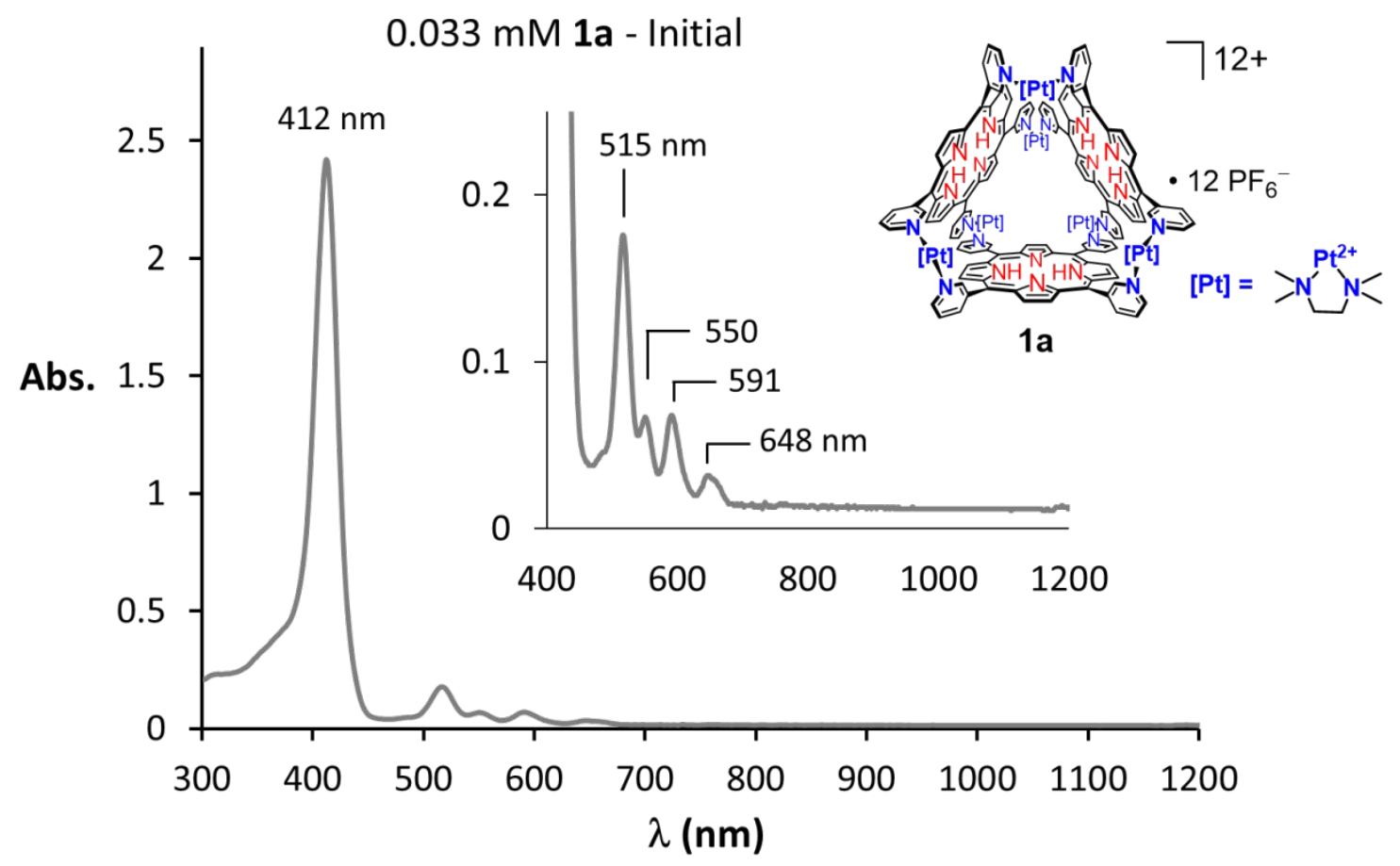

Figure S38: UV-Vis-NIR spectrum of $0.033 \mathrm{mM} 1 \mathrm{a}$ in its initial +12 state. The spectrum displays characteristic porphyrin features: Soret band (412 nm) and four Q peaks (515, 550, 591, $648 \mathrm{~nm})$. 


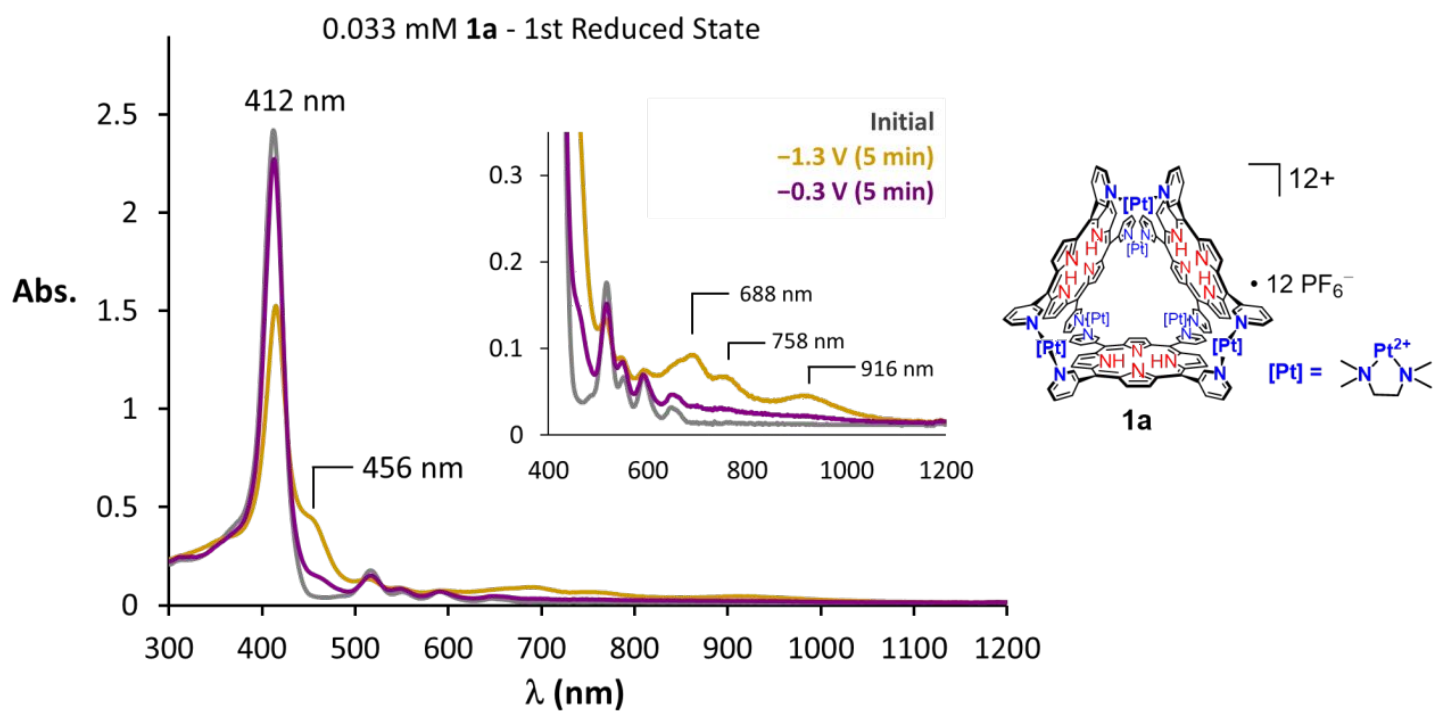

Figure S39: UV-vis-NIR spectra of $1 \mathrm{a}(0.033 \mathrm{mM})$ showing the initial spectrum of the +12 state (gray), the spectrum after applying a potential of $-1.3 \mathrm{~V}$ vs. $\mathrm{Fc}^{+/ 0}$ for 5 minutes (yellow), and the spectrum after reoxidation at $-0.3 \mathrm{~V}$ vs. $\mathrm{Fc}^{+/ 0}$ for 5 minutes (purple). The reduced species shows new absorbances at $\lambda_{\max }=456,688,758$, and $916 \mathrm{~nm}$, and a decrease in the intensity of the Soret band at $412 \mathrm{~nm}$, both indicating the reduction of the porphyrin walls to their radical anion states. ${ }^{18}$ After reoxidation, the Soret band and $Q$ peaks of the original spectrum were restored.

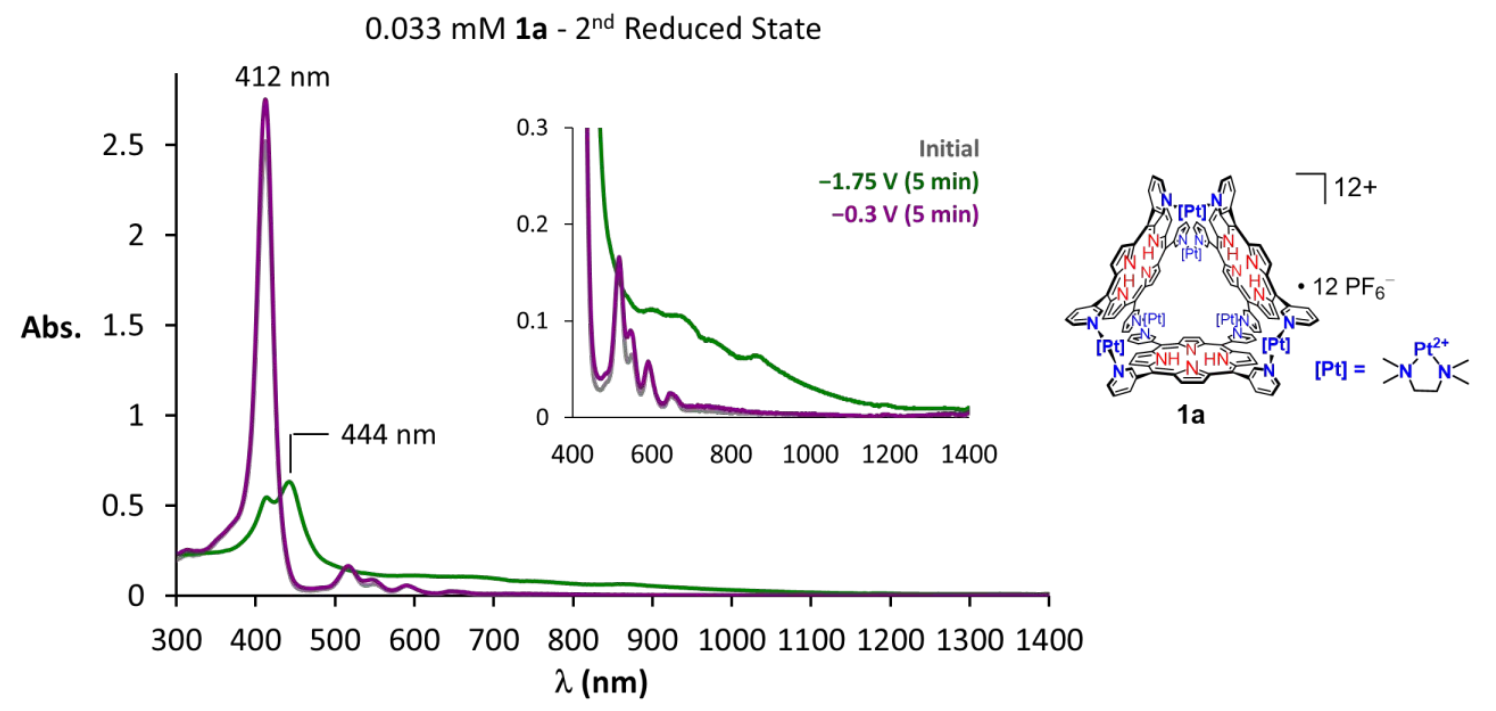

Figure S40: UV-vis-NIR spectra of $1 \mathrm{a}(0.033 \mathrm{mM})$ showing the initial spectrum of the +12 state (gray), the spectrum after applying a potential of $-1.75 \mathrm{~V} \mathrm{vs.} \mathrm{Fc}^{+/ 0}$ for 5 minutes (green), and the spectrum after reoxidation at $-0.3 \mathrm{~V}$ vs. $\mathrm{Fc}^{+/ 0}$ for 5 minutes (purple). The reduced species shows a new absorbance at $\lambda_{\max }=444 \mathrm{~nm}$ and nearly complete disappearance of the Soret band at 412 $\mathrm{nm}$. A broad absorption with poorly defined features extends from the NIR region into the visible region. After reoxidation, the Soret band and $Q$ peaks of the original spectrum were restored. 


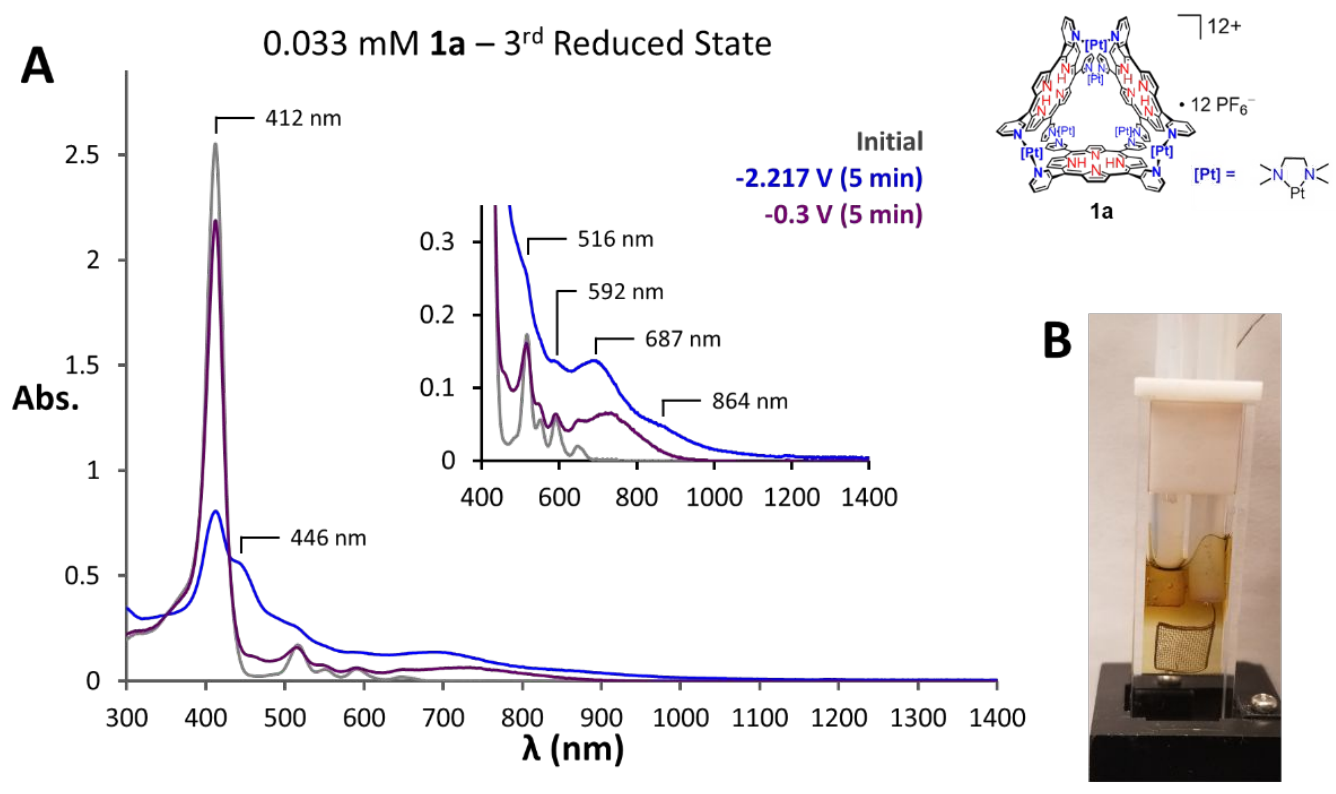

Figure S41: (A ) UV-vis-NIR spectra of 1a $(0.033 \mathrm{mM})$ showing the initial spectrum of the +12 state (gray), the spectrum after applying a potential of $-2.22 \mathrm{~V}$ vs. $\mathrm{Fc}^{+/ 0}$ for 5 minutes (blue), and the spectrum after reoxidation at $-0.3 \mathrm{~V}$ vs. $\mathrm{Fc}^{+/ 0}$ for 5 minutes (purple). Features of the +12 and +9 states of 1a were observed in the spectrum of the reduced species. (B) Photograph of SEC cell containing $0.033 \mathrm{mM} 1 \mathrm{a}$ after 5 minutes of reoxidation at $-0.3 \mathrm{~V}$.

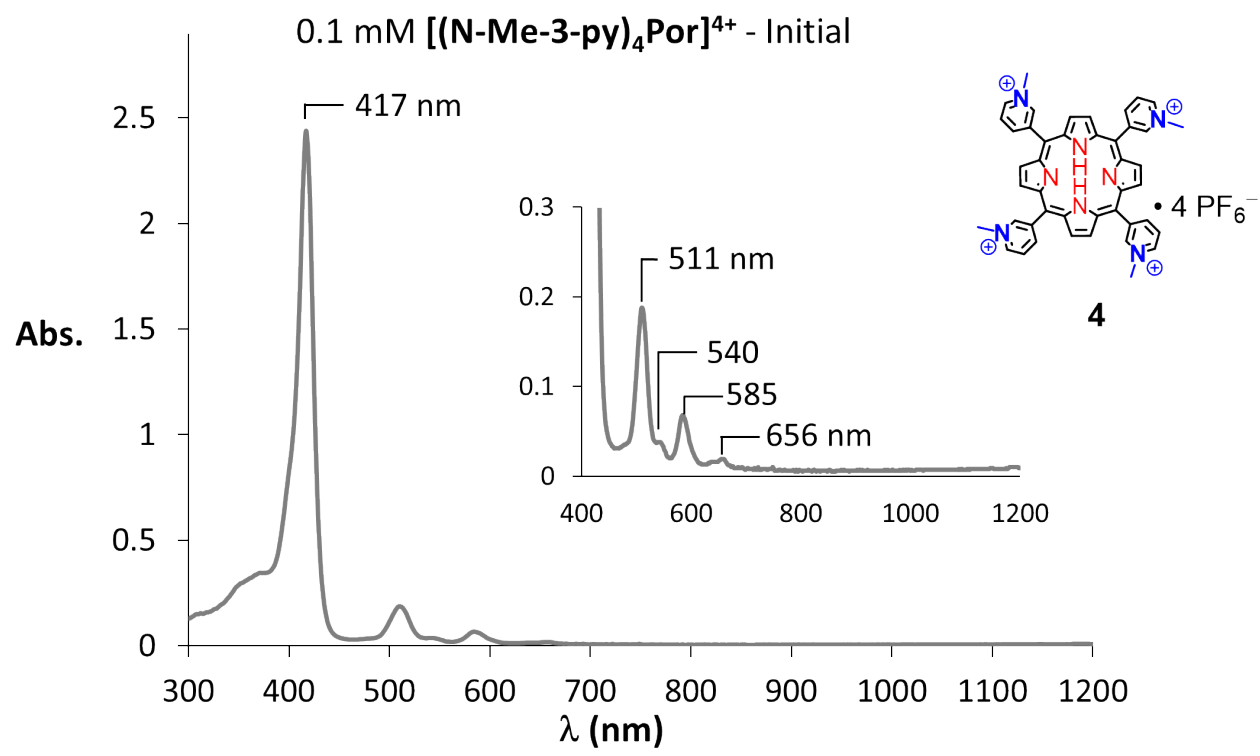

Figure S42: UV-Vis-NIR spectrum of $0.1 \mathrm{mM}$ [tetrakis(N-Me-3-pyridinium)porphyrin] $4 \mathrm{PF}_{6}(\mathbf{4}, 0.1$ $\mathrm{mM})$ in its initial +4 state. The spectrum displays characteristic porphyrin features: Soret band $(417 \mathrm{~nm})$ and four Q peaks $(511,540,585$, and $656 \mathrm{~nm})$. 


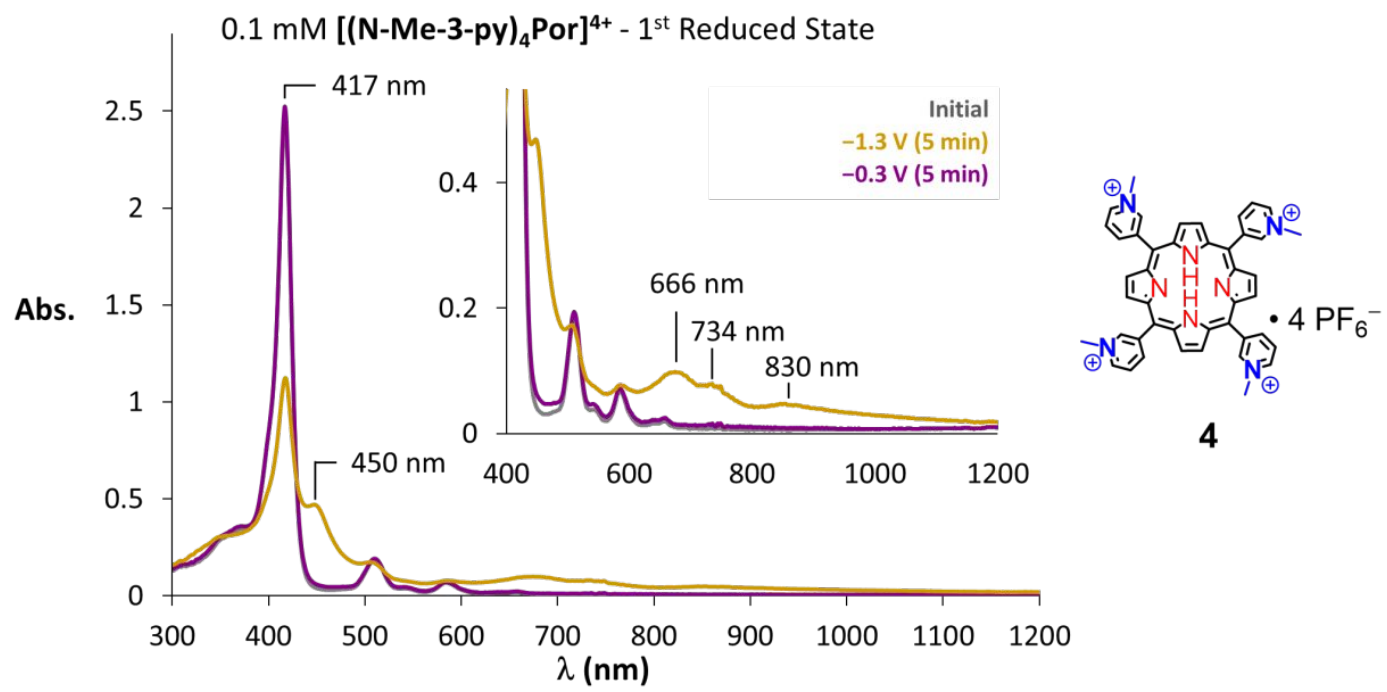

Figure S43: UV-vis-NIR spectra of $4(0.1 \mathrm{mM})$ showing the initial spectrum of the +4 state (gray), the spectrum after applying a potential of $-1.3 \mathrm{~V}$ vs. $\mathrm{Fc}^{+/ 0}$ for 5 minutes (yellow), and the spectrum after reoxidation at $-0.3 \mathrm{~V}$ vs. $\mathrm{Fc}^{+/ 0}$ for 5 minutes (purple). The reduced state of the porphyrin shows new absorbances at $\lambda_{\max }=450,666,734$, and $830 \mathrm{~nm}$, and a decrease in the intensity of the Soret band at $\lambda_{\max }=412 \mathrm{~nm}$, both indicating the reduction of the porphyrin to its radical anion state. ${ }^{18}$ The spectrum of this state of $\mathbf{4}$ is consistent with the first reduced states of $\mathbf{1 a , 1} \mathbf{1 b}$. Upon reoxidation, the Soret band and $Q$ peaks of the original spectrum were restored.

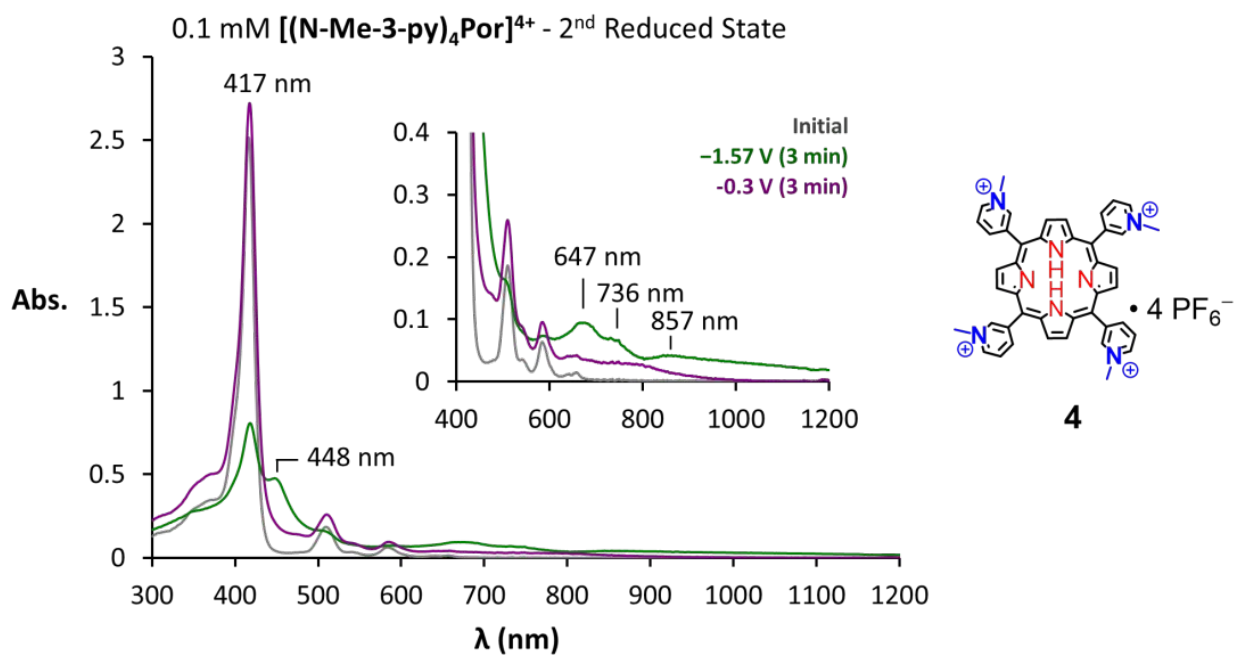

Figure S44: UV-vis-NIR spectra of $4(0.1 \mathrm{mM})$ showing the initial spectrum of the +4 state (gray), the spectrum after applying a potential of $-1.57 \mathrm{~V}$ vs. $\mathrm{Fc}^{+/ 0}$ for 3 minutes (green), and the spectrum after reoxidation at $-0.3 \mathrm{~V}$ vs. $\mathrm{Fc}^{+/ 0}$ for 3 minutes (purple). The reduced species shows new absorbances at 448,647, 736, and $857 \mathrm{~nm}$, and a ca. 75\% decrease of the Soret band at 417 $\mathrm{nm}$. After reoxidation, the Soret band and $\mathrm{Q}$ peaks of the original spectrum were restored. 


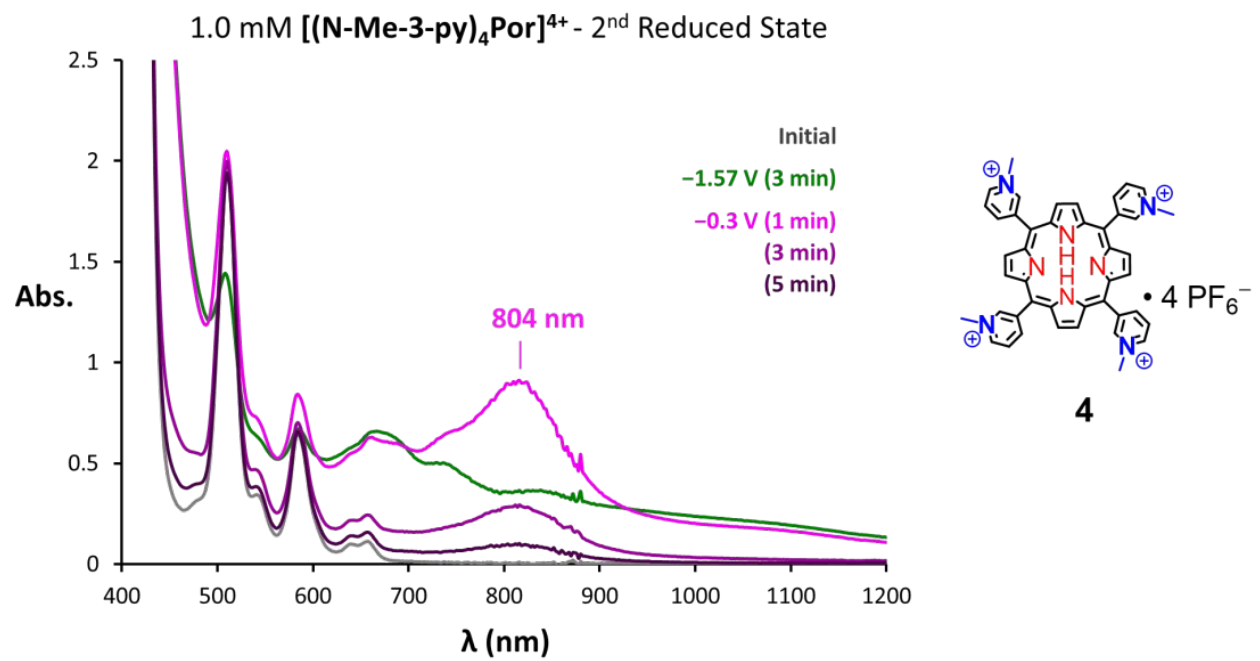

Figure S45: UV-vis-NIR spectra of a $1.0 \mathrm{mM}$ solution of 4 showing the initial spectrum of the +4 state (gray), the spectrum after applying a potential of $-1.57 \mathrm{~V} \mathrm{vs.} \mathrm{Fc}^{+/ 0}$ for $3 \mathrm{~min}$ (green), and the spectrum after reoxidation at $-0.3 \mathrm{~V}$ vs. $\mathrm{Fc}^{+/ 0}$ for $1 \mathrm{~min}$ (pink), $3 \mathrm{~min}$ (purple), and 5 min (dark purple). The reduced state (green) shows the same features as observed for this state at $0.1 \mathrm{mM}$ concentration of 4 (Figure S48), but upon applying an oxidizing potential, the $1.0 \mathrm{mM}$ sample exhibits an intense absorption at $\lambda_{\max }=804 \mathrm{~nm}$ that was not observed at lower concentrations. This broad feature is attributed to the aggregation of the porphyrin units, and after 5 minutes this feature disappears almost completely while the original spectrum of the +4 state is restored.

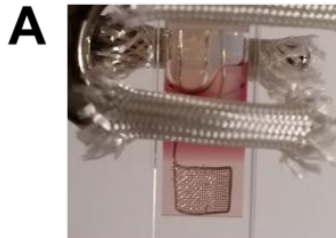

Initial

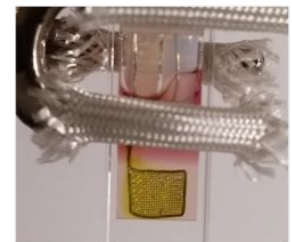

$-1.50 \mathrm{~V}$

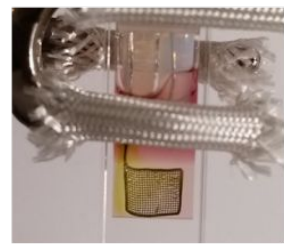

$-1.80 \mathrm{~V}$

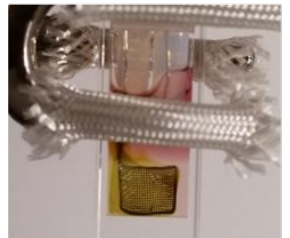

$-2.10 \mathrm{~V}$

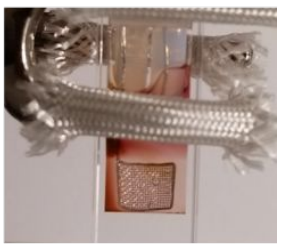

$-0.30 \mathrm{~V}$

B
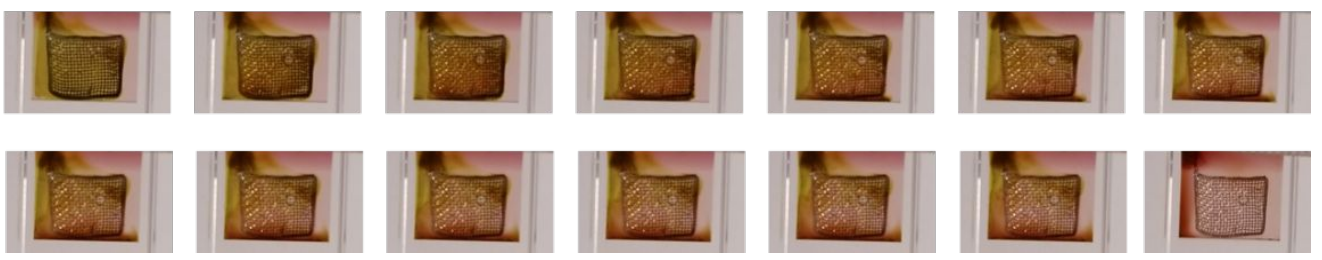

Figure S46. Monitoring the reduction of Cage $\mathbf{1 b}$ in the spectroelectrochemistry cuvette outside of the spectrometer: (A) Sequential reductions of the cage followed by reoxidation. The loss of color in solution and darkening of the Pt mesh electrode at $-1.80 \mathrm{~V}$ indicate the loss of solubility of the cage in its neutral charge state. The solution darkens at a more negative potential, showing the better solubility of the cage when it is further reduced. (B) Images taken every $10 \mathrm{~s}$ during reoxidation after reduction at $-2.10 \mathrm{~V}$. The red-purple color of the porphyrins is restored rapidly but sinks, possibly due to an increase in density as material adhered to the electrode re-enters solution. The final image skips ahead to $5 \mathrm{~min}$ to show complete restoration of the original color. 


\section{References}

1. Formiga, A. L. B.; Nogueira, A. F.; Araki, K.; Toma, H. E., Contrasting photoelectrochemical behaviour of two isomeric supramolecular dyes based on meso-tetra(pyridyl)porphyrin incorporating four ( $\mu 3-$ oxo)- triruthenium(iii) clusters. New Journal of Chemistry 2008, 32 (7), 1167-1174.

2. Berezin, M. B.; Berezina, N. M.; Bazanov, M. I.; V'yugin, A. I.; Semeikin, A. S.; Glazunov, A. V., The influence of the macroring structure on the enthalpies of solution of tetrapyridylporphyrin derivatives in water. Russian Journal of Physical Chemistry A 2010, 84 (8), 1449-1451.

3. Cusumano, M.; Di Pietro, M. L.; Giannetto, A., Relationship between binding affinity for calf-thymus DNA of $\left[\mathrm{Pt}\left(2,2^{\prime}-\mathrm{bpy}\right)(\mathrm{n}-\mathrm{Rpy}) 2\right] 2+(\mathrm{n}=2,4)$ and basicity of coordinated pyridine. Chemical Communications 1996, (22), 2527-2528.

4. Rotondo, E.; Bruschetta, G.; Bruno, G.; Rotondo, A.; Di Pietro, M. L.; Cusumano, M., NMR Investigation and Dynamic Behaviour of [2,2'-Bipyridylbis(pyridine)platinum(II)]2+ and Related Cationic Complexes - Crystal Structure of [Pt(bipy)(py)2](PF6)2. European Journal of Inorganic Chemistry 2003, (14), 2612-2618.

5. Bar, A. K.; Mohapatra, S.; Zangrando, E.; Mukherjee, P. S., A Series of Trifacial Pd6 Molecular Barrels with Porphyrin Walls. Chemistry - A European Journal 2012, 18 (31), 9571-9579.

6. Gushchin, P. V.; Tyan, M. R.; Bokach, N. A.; Revenco, M. D.; Haukka, M.; Wang, M.-J.; Lai, C.-H.; Chou, P.-T.; Kukushkin, V. Y., Novel Tailoring Reaction for Two Adjacent Coordinated Nitriles Giving Platinum 1,3,5-Triazapentadiene Complexes. Inorganic Chemistry 2008, 47 (24), 11487-11500.

7. Collins, J.; Gillard, R. D.; Hursthouse, M. B., An unusual lability of 2,2'-bipyridyl chelated to platinum(ii). Polyhedron 1993, 12 (2), 255-257.

8. Field, J. S.; Haines, R. J.; Summerton, G. C., Synthesis and crystal structure determination of the triflate salt of diacetonitrile(2,2'-bipyridine)platinum(II). Journal of Coordination Chemistry 2003, 56 (13), 1149-1155.

9. Zoski, C. G., Handbook of Electrochemistry. 1st ed.; Elsevier: Amsterdam, 2007.

10. Dolbier, W. R., Guide to Fluorine NMR for Organic Chemists. Wiley: Hoboken, NJ, 2008.

11. Allouche, L.; Marquis, A.; Lehn, J.-M., Discrimination of Metallosupramolecular Architectures in Solution by Using Diffusion Ordered Spectroscopy (DOSY) Experiments: Double-Stranded Helicates of Different Lengths. Chemistry - A European Journal 2006, 12 (28), 7520-7525.

12. Sheldrick, G. M. SADABS - Bruker Nonius Area Detector Scaling and Absorption Correction, Bruker-AXS Inc.: Madison, Wisconsin, 2003.

13. Sheldrick, G. M., A short history of SHELX. Acta crystallographica. Section A, Foundations of crystallography 2008, 64 (Pt 1), 112-22.

14. Sheldrick, G. M. SHELXL97, Program for Crystal Structure Refinement, University of Göttingen: Germany, 1997.

15. Spek, A. L., checkCIF validation ALERTS: what they mean and how to respond. Acta Crystallographica Section E 2020, 76, 1-11.

16. Gee, C. T.; Arntson, K. E.; Urick, A. K.; Mishra, N. K.; Hawk, L. M. L.; Wisniewski, A. J.; Pomerantz, W. C. K., Protein-observed 19F-NMR for fragment screening, affinity quantification and druggability assessment. Nature Protocols 2016, 11 (8), 1414-1427.

17. Furutani, Y.; Kandori, H.; Kawano, M.; Nakabayashi, K.; Yoshizawa, M.; Fujita, M., In Situ Spectroscopic, Electrochemical, and Theoretical Studies of the Photoinduced Host-Guest Electron Transfer that Precedes Unusual Host-Mediated Alkane Photooxidation. Journal of the American Chemical Society 2009, 131 (13), 4764-4768.

18. Cui, Y.; Zeng, L.; Fang, Y.; Zhu, J.; Xu, H.-J.; Desbois, N.; Gros, C. P.; Kadish, K. M., Electrochemical and Spectroelectrochemical Properties of Free-Base Pyridyl- and N-Alkyl-4-Pyridylporphyrins in Nonaqueous Media. ChemElectroChem 2016, 3 (1), 110-121. 